\title{
WestVirginiaUniversity
}

THE RESEARCH REPOSITORY @ WVU

Graduate Theses, Dissertations, and Problem Reports

2019

\section{Short-term abstinence effects in experienced electronic cigarette users}

Nicholas J. Felicione

njfelicione@mix.wvu.edu

Follow this and additional works at: https://researchrepository.wvu.edu/etd

Part of the Biological Psychology Commons, Cognition and Perception Commons, and the Health Psychology Commons

\section{Recommended Citation}

Felicione, Nicholas J., "Short-term abstinence effects in experienced electronic cigarette users" (2019). Graduate Theses, Dissertations, and Problem Reports. 7472.

https://researchrepository.wvu.edu/etd/7472

This Dissertation is protected by copyright and/or related rights. It has been brought to you by the The Research Repository @ WVU with permission from the rights-holder(s). You are free to use this Dissertation in any way that is permitted by the copyright and related rights legislation that applies to your use. For other uses you must obtain permission from the rights-holder(s) directly, unless additional rights are indicated by a Creative Commons license in the record and/ or on the work itself. This Dissertation has been accepted for inclusion in WVU Graduate Theses, Dissertations, and Problem Reports collection by an authorized administrator of The Research Repository @ WVU.

For more information, please contact researchrepository@mail.wvu.edu. 
Short-term abstinence effects in experienced electronic cigarette users

Nicholas J. Felicione, M.S.

Dissertation submitted

to the Eberly College of Arts and Sciences

at West Virginia University

in partial fulfillment of the requirements for the degree of

Doctor of Philosophy in

Psychology

\author{
Melissa Blank, Ph.D., Chair \\ Kevin Larkin, Ph.D. \\ James Mahoney III, Ph.D. \\ Francis McClernon, Ph.D. \\ Cole Vonder Haar, Ph.D.
}

Department of Psychology

Morgantown, West Virginia

2019

Keywords: electronic cigarette, abstinence, nicotine withdrawal, nicotine dependence, cognition Copyright 2019 Nicholas J. Felicione 


\begin{abstract}
Short-term abstinence effects in experienced electronic cigarette users
\end{abstract}

Nicholas J. Felicione

Nicotine/tobacco dependence is a problem that negatively affects health at an individual and population level. Nicotine/tobacco dependence may be best assessed by measuring withdrawal symptoms of a nicotine/tobacco user who abstains from use. Withdrawal symptoms experienced by cigarette smokers are well characterized, including deficits in attention and memory, as well as self-reported cravings, urges, and other symptoms. While withdrawal in cigarette smokers has been studied extensively, little is known about dependence and withdrawal associated with newer products, such as electronic cigarettes (ECIGs). ECIGs can deliver nicotine comparably to tobacco cigarettes, indicating that they may have the potential to cause dependence. However, extant work assessing ECIG dependence and withdrawal is confounded by current or previous cigarette smoking in ECIG users. Thus, the purpose of this project was to characterize cognitive, subjective, and physiological measures of ECIG withdrawal in ECIG users who are not regular cigarette smokers. Eleven ECIG users that were primarily nonsmokers participated in two, 3.5hour sessions that differed by ECIG use: 180 minutes ad lib ECIG use and 180 minutes abstinent. Outcome measures included subjective questionnaires of withdrawal and nicotine effects, heart rate, cognitive assessments of sustained attention, working memory, and inhibitory control, and ECIG reward. Participants subjectively reported more craving, urges, and desire to vape at 120 to 180 minutes of abstinence compared to baseline. Participants also had poorer accuracy on an inhibitory control task at 120 minutes of abstinence compared to baseline abstinence or 120 minutes of ad lib ECIG use. Additional analyses included assessing characteristics that predict ECIG dependence, characterizing ad lib puff topography, and predicting withdrawal based on puff topography. Higher nicotine concentrations, daily ECIG use, longer durations of ECIG use, and higher wattages were associated with increased nicotine dependence. Results of this study demonstrate preliminary evidence of short-term withdrawal in ECIG users that are primarily nonsmokers, though findings are limited by a small sample size. Future research should be continue with similar experimental designs but increase sample size and assess longer durations of abstinence to better characterize ECIG withdrawal and dependence. 


\section{Acknowledgements}

The completion of this dissertation project would not have been successful without the contribution and support of multiple people. I would like to thank my advisor, Dr. Melissa Blank, for her training, guidance, and unconditional support throughout this project and my graduate career. I appreciate the useful suggestions and feedback to improve the project from my committee members, Dr. Kevin Larkin, Dr. James Mahoney III, Dr. F. Joseph McClernon, and Dr. Cole Vonder Haar. Additional thanks go to Drs. McClernon and Mahoney for their expertise and assistance regarding cognitive assessments and programming of tasks. Finally, I would like to thank my lab mates who provided help on this project, Ilana Haliwa and Dani Kisner. Dani contributed many hours of work into video scoring for this project, and I appreciate her commitment to this task.

This work was supported by the West Virginia University Eberly College of Arts and Sciences / Department of Psychology Doctoral Research Award. 
Table of Contents

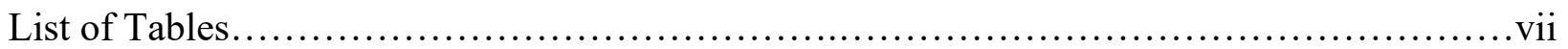

List of Figures..................................................................

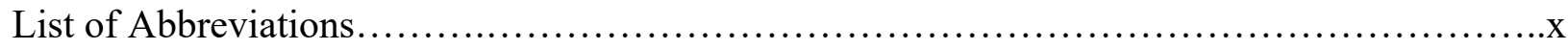

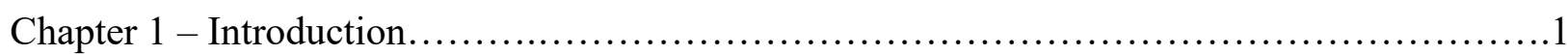

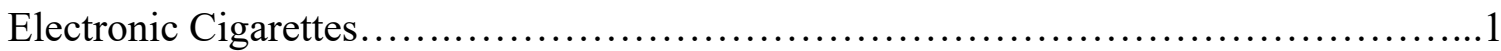

Nicotine Dependence........................................................4

Nicotine Withdrawal..........................................................

Subjective reports of nicotine withdrawal ................................6

Cognitive measures of nicotine withdrawal............................... 7

Sustained attention............................................. 8

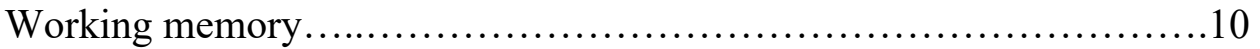

Inhibitory control............................................... 12

ECIG Dependence and Withdrawal............................................. 14

Statement of the Problem......................................................... 18

Clinical and Regulatory Implications.......................................... 18

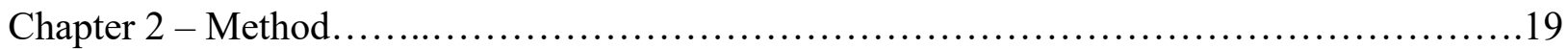

Selection of Participants..................................................... 19

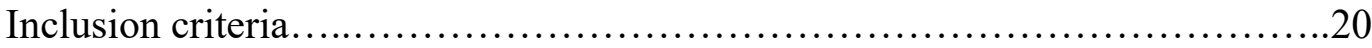

Exclusion criteria...................................................21

Telephone screening procedure.......................................21

Informed consent $\&$ in-person screening procedures.........................22

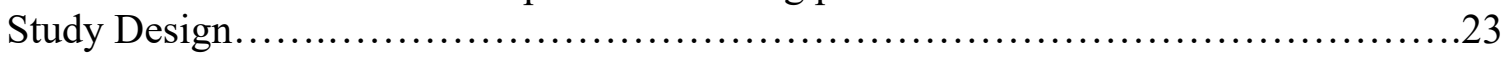

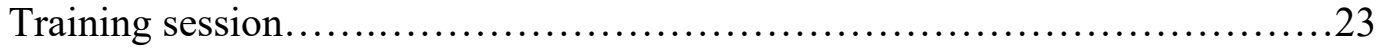

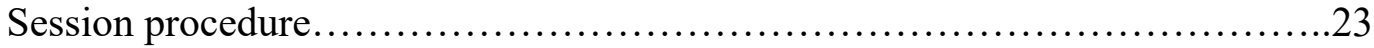

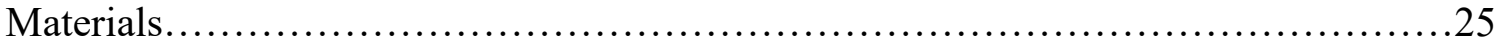

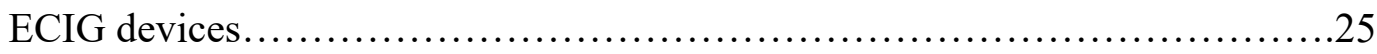

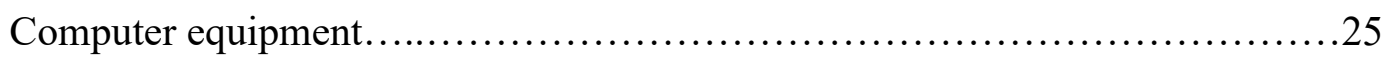

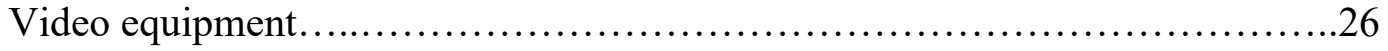

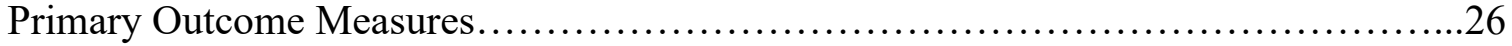

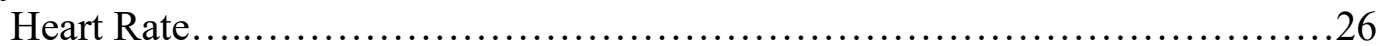

Minnesota nicotine withdrawal scale....................................27

Tiffany-Drobes questionnaire of smoking urges: brief form .................. 27

Direct effects of nicotine scale......................................... 28

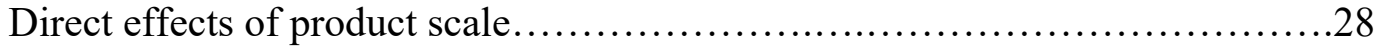

ECIG dependence and use............................................29

Secondary Outcome Measures....................................................29

Rapid visual information processing task ...............................29

$N$-back task ......................................................... 30

Cued go/no-go task................................................... 32

Multiple choice procedure............................................33 


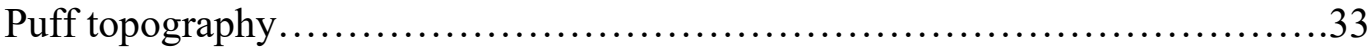

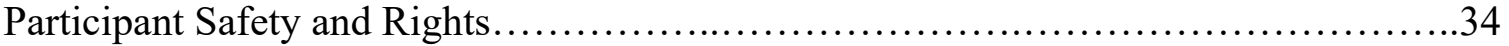

Data Preparation and Analysis.......................................................

Data preparation............................................................ 35

Feasibility and recruitment............................................ 35

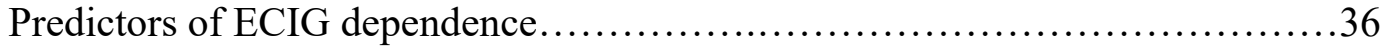

Primary outcomes....................................................... 37

Secondary outcomes...................................................... 38

Puff topography............................................... 38

Predicting withdrawal from puff topography........................39

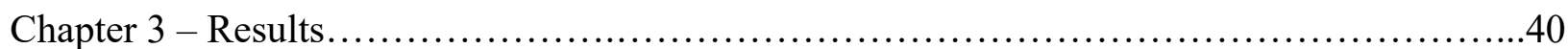

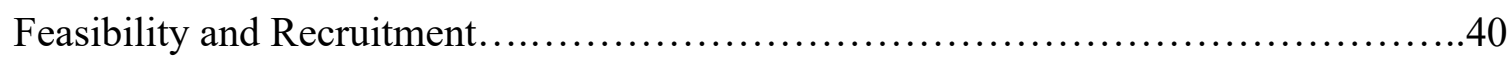

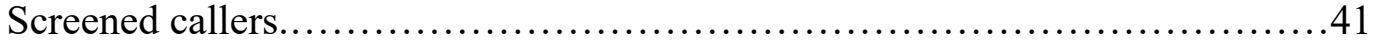

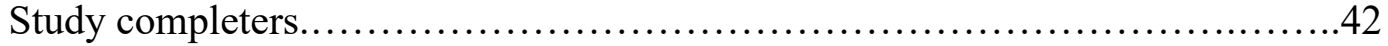

Predictors of Dependence..................................................... 42

Heart Rate...................................................................44

Subjective Questionnaires..................................................44

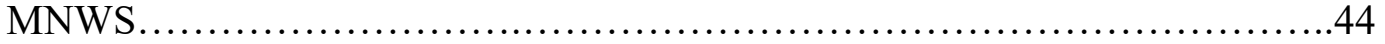

Tiffany-Drobes QSU-Brief..........................................45

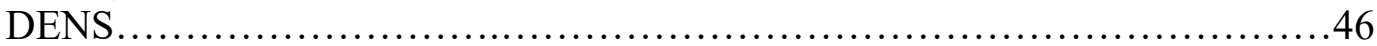

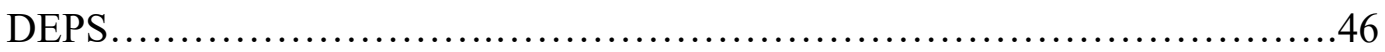

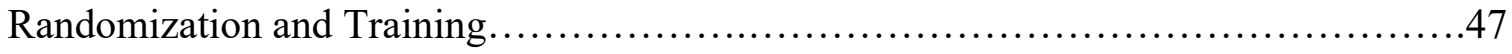

Cognitive Tasks. ..........................................................47

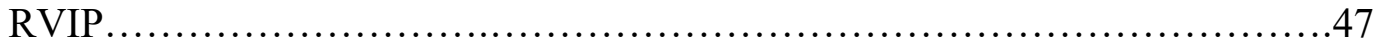

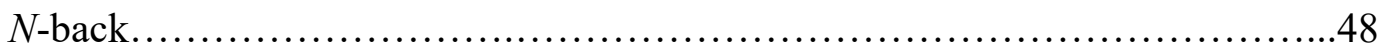

Cued Go/No-Go..................................................... 48

Order Effects.......................................................... 48

Multiple Choice Procedure ....................................................... 48

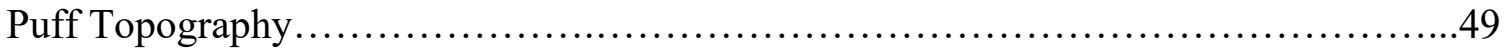

Predicting Withdrawal from Topography.......................................50

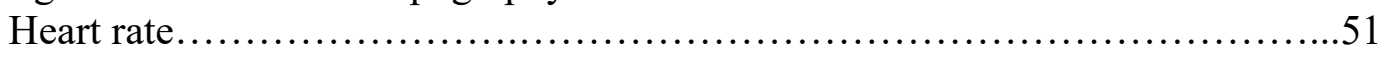

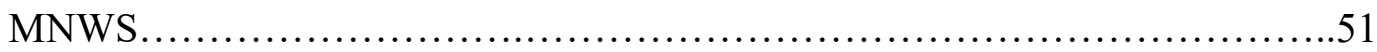

Tiffany-Drobes QSU-Brief.............................................52

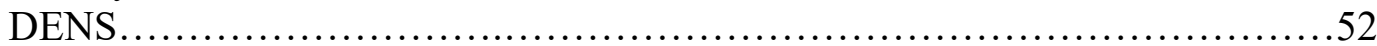

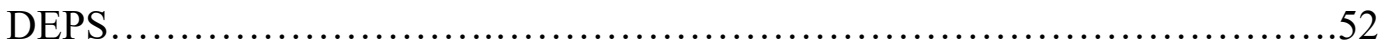

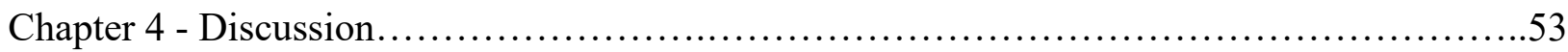

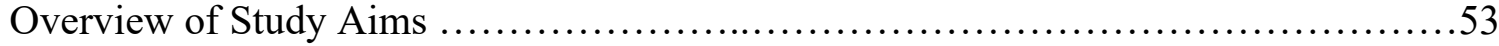

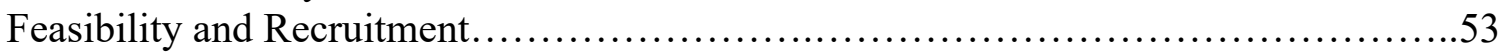

Predictors of ECIG Dependence..................................................56

Abstinence Effects on Primary and Secondary Outcomes.............................58

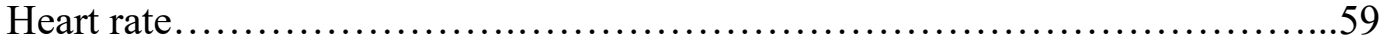

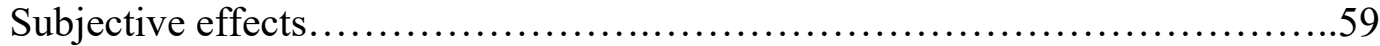

Cognitive effects..................................................61 


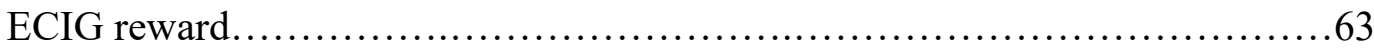

Puff Topography During 180 Minutes of ad lib ECIG Use...............................64

Predicting Withdrawal From Puff Topography.....................................66

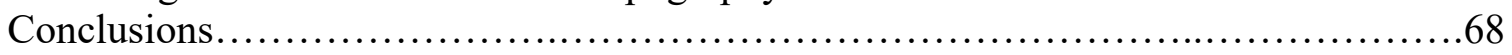

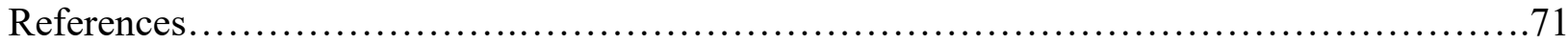

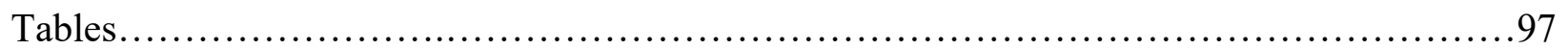

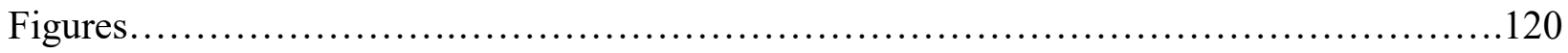

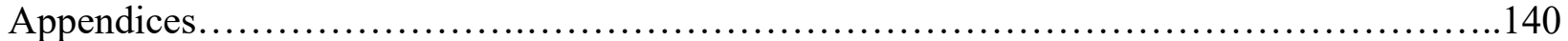

Appendix A: Telephone Screening Questionnaire ................................140

Appendix B: Demographic Information......................................... 145

Appendix C: Health and Drug Use Form.........................................146

Appendix D: MINI Tobacco Use Disorder Screen for ECIGs..........................149

Appendix E: Minnesota Nicotine Withdrawal Scale.................................152

Appendix F: Questionnaire of Smoking Urges-Brief............................. 153

Appendix G: Direct Effects of Nicotine Scale......................................155

Appendix H: Direct Effects of Product Scale .......................................156

Appendix I: Multiple Choice Procedure ................................................157

Appendix J: ECIG Dependence Predictor Variables.................................158 


\section{List of Tables}

Table 1. Diagnostic Criteria for Tobacco Use Disorder.

Table 2. Diagnostic Criteria for Tobacco Withdrawal.

Table 3. Mean (SD) or percentage for demographic, cigarette use, and ECIG use characteristics of all callers, ineligible callers, and completers.

Table 4. Mean (SD) or percentage for additional characteristics measured and in-person screening for completers.

Table 5. Descriptive statistics for variables considered for multiple linear regression to predict

PSECDI scores.

Table 6. Multiple linear regression models to predict PSECDI scores.

Table 7. Repeated-measures ANOVA results for heart rate and subjective outcomes

Table 8. Mean (SD) for best cognitive performance during training 106

Table 9. Paired-samples $t$-test results comparing 120 minutes ECIG abstinence to baseline abstinence and 120 minutes ad lib ECIG use.

Table 10. Mean (SE) cognitive performance for the ad lib and abstinent conditions at baseline and 120 minutes. 108

Table 11. Mean (SD) change in cognitive performance from baseline to 120 minutes for the $a d$ $l i b$ and abstinent conditions .

Table 12. Mean (SD) cognitive performance for session 1 and session 2 at baseline and 120 minutes

Table 13. Mean (SE) MCP crossover point for the $a d$ lib and abstinence condition at baseline, 75 minutes, and 150 minutes.

Table 14. Puff topography clustering patterns for each participants during the ad lib condition.

Table 15. Multiple linear regressions to predict withdrawal based on puff topography 


\section{List of Figures}

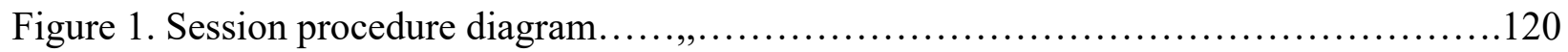

Figure 2. Rapid Visual Information Processing task diagram...........................121

Figure 3. $N$-back task diagram.................................................. 122

Figure 4. Cued Go/No-Go Task diagram......................................... 123

Figure 5. Diagram of recruitment, enrollment, reasons for ineligibility, nonattendance, and

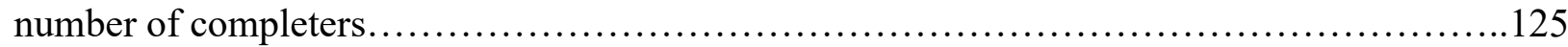

Figure 6. Mean heart rate during 180 minutes of ad lib ECIG use or ECIG abstinence.........126

Figure 7. Mean ratings for MNWS craving during 180 minutes of ad lib ECIG use or ECIG abstinence.

Figure 8. Mean ratings for QSU-Brief Factor 1 (intention to smoke) during 180 minutes of $a d$ lib

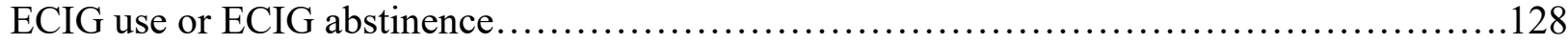

Figure 9. Mean ratings for DENS weak during 180 minutes of ad lib ECIG use or ECIG abstinence.

Figure 10. Mean ratings for DEPS pleasant during 180 minutes of ad lib ECIG use or ECIG abstinence.

Figure 11. Mean RVIP percent correct for $a d \mathrm{lib}$ and abstinent conditions at baseline and 120 minutes

Figure 12. Mean 3-back accuracy for $a d$ lib and abstinent conditions at baseline and 120 minutes.

Figure 13. Mean No-Go incongruent accuracy for $a d$ lib and abstinent conditions at baseline and 120 minutes...................................................................... 133

Figure 14. Puffing patterns in the ad lib condition for participant $501 \ldots \ldots \ldots \ldots \ldots \ldots \ldots \ldots \ldots . \ldots \ldots$

Figure 15. Puffing patterns in the ad lib condition for participant $507 \ldots \ldots \ldots \ldots \ldots \ldots \ldots \ldots \ldots$

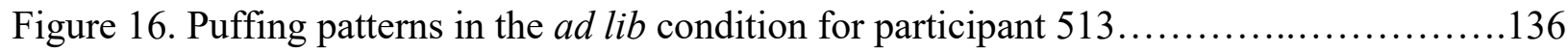

Figure 17. Puffing patterns, heart rate, and MNWS urges for participant 501 in the ad lib condition. 
Figure 18. Puffing patterns, heart rate, and MNWS urges for participant 507 in the ad lib

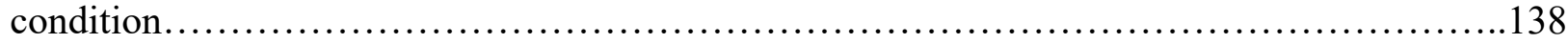

Figure 19. Puffing patterns, heart rate, and MNWS urges for participant 513 in the ad lib

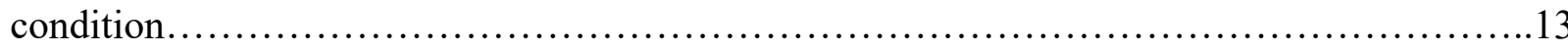


List of Abbreviations

AIC

ANOVA

APA

$\mathrm{B}$

$\beta$

DENS

DEPS

ECIG

FDR

HSD

IPI

M

MCP

$\mathrm{mg} / \mathrm{ml}$

$\min$

MINI

$\mathrm{ml}$

MNWS

ms

PSECDI

QSU-Brief

RVIP
Akaike information criterion

Analysis of variance

American Psychiatric Association

Beta (unstandardized)

Beta (standardized)

Direct Effects of Nicotine Scale

Direct Effects of Product Scale

Electronic cigarette

False discovery rate

Honestly significant difference

Interpuff interval

Mean

Multiple Choice Procedure

milligrams per milliliter

minutes

Mini-International Neuropsychiatric Interview

milliliter

Minnesota Nicotine Withdrawal Scale

milliseconds

Penn State Electronic Cigarette Dependence Index

Tiffany Drobes Questionnaire of Smoking Urges-Brief

Rapid Visual Information Processing 
SD

$\sec$

SE

VAS

VIF
Standard deviation

seconds

Standard error of the mean

Visual analog scale

Variance Inflation Factor 


\section{Chapter 1 - Introduction}

\section{Electronic Cigarettes}

Since becoming commercially available around 2007, the popularity of electronic cigarettes (ECIGs) has increased dramatically. By 2010, ECIGs were more commonly searched on the internet than tobacco products such as snus or nicotine replacement therapies (Ayers, Ribisl, \& Brownstein, 2011). From 2010 to 2013, ever use of ECIGs among adults increased from below 2\% to approximately 13\% (McMillen, Gottlieb, Shaefer, Winickoff, \& Klein, 2015). This increase in prevalence is consistent across most demographic groups, including both sexes, most age groups, and former and current smokers (King, Patel, Nguyen, \& Dube, 2015). Recent national data also show that approximately $13 \%$ of adults have ever used ECIGs, as well as that 3.7\% - 4.5\% currently use ECIGs (Mirbolouk et al., 2018; Schoenborn \& Gindi, 2015; Wilson \& Wang, 2017). ECIGs are now the second most commonly used nicotine product (cigarettes being the most common), surpassing alternative tobacco products such as cigars and smokeless tobacco (Phillips et al., 2017). Additionally, ECIGs are more commonly used in younger generations, with over $20 \%$ of adults aged 18-24 having ever tried an ECIG and use consistently declining with increasing age (Schoenborn \& Gindi, 2015). Rates of lifetime ECIG use are higher in current ( $\sim 47 \%)$ and former $(\sim 55 \%)$ cigarette smokers than never cigarette smokers $(3.2 \%)$ (Schoenborn \& Gindi, 2015). Similarly, rates of current use of ECIGs (i.e., every day or some days) are close to $16 \%$ for current cigarette smokers, $22 \%$ for former cigarette smokers, and $0.4 \%$ for never cigarette smokers (Schoenborn \& Gindi, 2015). Also notable is that lifetime and current ECIG use is more likely among cigarette smokers who have made a quit attempt in the past year, relative to cigarette smokers who did not make an attempt (Schoenborn \& Gindi, 2015). 
ECIGs are a class of electronic nicotine delivery devices, or battery-operated products intended to delivery nicotine and other flavorants to a user (Brandon et al., 2015; Brown \& Cheng, 2014). They all share some common features: battery, heating element, storage component, and liquid solution (Brown \& Cheng, 2014). The liquid solution typically contains nicotine, solvents (propylene glycol and vegetable glycerin), and flavorants. In most cases, the battery will be activated by a flow sensor upon puffing or a manual press of a button. The battery provides power to the heating element such as a coil, which contains a wick to soak up the solution and an atomizer that aerosolizes the solution. Finally, the user inhales the aerosol through the mouthpiece. Still, ECIG designs and features have changed tremendously since their emergence, and there remains enormous variety in the currently available products. For example, $1^{\text {st }}$ generation devices (also known as "cig-alikes") model the design of a cigarette with a mouthpiece that resembles a cigarette filter and an LED light that resembles the burning end of a cigarette rod (Brown \& Cheng, 2014). These devices are pre-filled with nicotine solution and are disposable after use. More modern products no longer retain a cigarette-like resemblance. These $2^{\text {nd }}$ generation ECIGs (also known as "tank" models) typically hold the solution in a tank with or without an atomizer, and individuals can refill the solution as needed. Even more recently, a slew of $3^{\text {rd }}$ generation ECIGs (also known as "mods") have emerged, allowing individuals to build their own atomizers, as well as manipulate the battery voltage and atomizer resistance of the ECIG (Brandon et al., 2015; Farsalinos et al., 2014). Newer $4^{\text {th }}$ generation (also known as "pods" or "pod mods") have gained popularity, that typically contain high concentrations of nicotine salts (Eissenberg et al., 2018). These designs also allow the user more freedom to choose the nicotine content, solvents, and flavorants of their solution. Such ECIG models allow the user 
great control over product features, which results in a greater user influence on the nicotine yield of the device.

Indeed, the level of nicotine delivered to the user is influenced by both features of the ECIG design and behaviors of the user. As for design features, some ECIGs deliver little to no nicotine to the user while other ECIGs deliver levels of nicotine that surpass that for a cigarette (Shihadeh \& Eissenberg, 2015). Those better able to deliver nicotine tend to have increased battery power (Kosmider, Sobczak, Knysak, \& Goniewicz, 2014; Talih et al., 2015; Wagener et al., 2017), and thus later generation models are more efficient delivery devices than the $1^{\text {st }}$ generation (Bullen et al., 2010; Vansickel, Cobb, Weaver, \& Eissenberg, 2010). It is not surprising, therefore, that many ECIG users report switching from the cig-alike to more advanced models (Yingst et al., 2015). Of course, increasing the nicotine concentration of the liquid solution also results in increased nicotine delivery to the user (Hiler et al., 2017; Ramôa et al., 2016; Talih et al., 2015), as may changing the ratio of the solvents propylene glycol and vegetable glycerin (Kosmider et al., 2014). Yet even when the device features and liquid ingredients are held constant, the levels of nicotine delivered may differ substantially between ECIG users. Such differences are likely due to users' puffing behavior, including their number, duration, and volume of puffs. Experienced ECIG users have shown to take puffs that are approximately twice as long as ECIG-naïve cigarette smokers (Farsalinos, Romagna, Tsiapras, Kyrzopoulous, \& Voudris, 2013a), and longer puff durations are demonstrated to result in increased nicotine delivery (Hiler et al., 2017; Talih et al., 2015). Thus, the combination of the optimal device and users' response to that device likely results in ECIGs that have the nicotinedelivery capacity to produce cigarette-like, or potentially greater, levels of nicotine dependence (Ramôa et al., 2016; Shihadeh \& Eissenberg, 2015). Given that nicotine is a dependence- 
producing drug (Benowitz, 2010), the ability of ECIGs to effectively deliver nicotine has important implications for individual and public health. Thus, it is essential to begin understanding nicotine dependence in the context of ECIG use, focusing on individuals that are ECIG users.

\section{Nicotine Dependence}

Nicotine/tobacco dependence is a recognized medical condition in the Diagnostic and Statistical Manual of the American Psychiatric Association (APA; APA, 2013) as well as the International Classification of Diseases (World Health Organization, 1992). According to the APA (2013), tobacco use disorder is defined as "A problematic pattern of tobacco use leading to clinically significant impairment or distress," (p. 571) including criteria such as tolerance and withdrawal (see Table 1 for full criteria; APA, 2013). Tolerance is defined as a need for increased amounts of tobacco to achieve the same effect, or a reduced effect with continued use of the same amount of tobacco. For example, non-cigarette smokers exposed to nicotine experience aversive effects including dizziness and nausea at lower nicotine doses than cigarette smokers, suggesting that cigarette smokers may have become tolerant to, or need increased amounts of nicotine to experience, these adverse effects (Pomerleau, 1995). A similar trend is seen for positive effects (e.g., head rush, arousal, vigor), even when controlling for differences in plasma nicotine concentration between smokers and non-smokers (Perkins, Epstein, Grobe, \& Fonte, 1994). Similarly, non-cigarette smokers show greater increases in heart rate and systolic blood pressure in response to nicotine than cigarette smokers (Pomerleau, 1995). Along with subjective and physiological tolerance, there is evidence of pharmacokinetic tolerance, with noncigarette smokers revealing higher plasma nicotine concentrations than cigarette smokers after acute nicotine administration (Srivastava, Russell, Feyerabend, Masterson, \& Rhodes, 1991). 
Still, while tolerance to nicotine is a criterion for tobacco dependence, it is considered a relatively weak indicator of dependence (Perkins, 2002). This weak relationship is evidenced by the lack of differential tolerance between dependent and non-dependent smokers, and the lack of association between tolerance and cessation success (Perkins et al., 2001; Perkins et al., 2002). For example, it might be expected that those smokers more tolerant to nicotine would be the least likely to quit smoking successfully, because they would be those more dependent on nicotine. However, research does not support this idea, rather demonstrating that tolerance may be unrelated to post-cessation withdrawal and relapse (Perkins et al, 2002).

Withdrawal, in contrast, is a particularly important marker of dependence. The criterion of withdrawal is defined as experiencing a characteristic tobacco withdrawal syndrome or using tobacco/nicotine to relieve or avoid that withdrawal syndrome (APA, 2013). Table 2 demonstrates the APA (2013) diagnostic criteria for tobacco withdrawal. As shown in this table, cigarette smokers often report the following symptoms of withdrawal experienced during a period of abstinence: cravings, difficulty concentrating, drowsiness, irritability, moodiness, and depression (Etter, 2005; Hughes \& Hatsukami, 1986; Welsch et al., 1999). These aversive effects may drive smokers to use cigarettes or other nicotine/tobacco products to relieve or avoid withdrawal symptoms. For example, nicotine replacement products (e.g., nicotine patch, nicotine gum) are used to deliver nicotine to the user to mitigate aversive effects during a cessation attempt, and the dose of nicotine delivered can be slowly decreased over time as the cigarette smoker transitions to complete abstinence (Stead et al., 2012). In one study, the use of a nicotine patch resulted in increased positive symptoms (e.g., positive affect) and decreased negative symptoms (e.g., negative affect, craving, difficulty concentrating) which were related to a significantly lower risk of relapse following cessation among smokers (Ferguson, Shiffman, \& 
Gwaltney, 2006). Additionally, smokers who report withdrawal effects such as anger, depressed mood, and craving are observed to have a shorter time to relapse following cessation (Swan, Ward, \& Jack, 1996). These and other data (Baker, Breslau, Covey, \& Shiffman, 2012; Ferguson et al., 2006; Swan et al., 1996) support the idea that the severity of tobacco withdrawal is a significant predictor of nicotine/tobacco dependence. Consequently, it is important to consider the phenomenon of withdrawal as an indicator of ECIG dependence.

\section{Nicotine Withdrawal}

Nicotine/tobacco withdrawal symptoms can be characterized using a variety of measures, which capture the subjective (Etter, 2005; Hughes \& Hatsukami, 1986), physiological (Hendricks, Ditre, Drobes, \& Brandon, 2006), and cognitive/behavioral (Ashare, Falcone, \& Lerman, 2014; Heishman, Taylor, \& Henningfield, 1994; Hughes, 2007b, McClernon, Addicott, \& Sweitzer, 2015) experiences of smokers. Given the wide variation in the symptoms experienced by product users, studies that include all of these measures of are the most complete way to characterize withdrawal, and ultimately, understand dependence (Hughes, 2007b). Some of the most common methods for measuring nicotine/tobacco withdrawal are described below.

Subjective reports of withdrawal. One of the most common methods for measuring withdrawal symptoms are subjective measures, or self-reports (for, review, see Hughes, 2007b), such as the Minnesota Nicotine Withdrawal Scale (MNWS; Hughes \& Hatsukami, 1986), the Cigarette Withdrawal Scale (Etter, 2005), and the Shiffman-Jarvik Withdrawal Scale (Shiffman \& Jarvik, 1976). While these measures differ in measurement scale type (e.g., visual analog, Likert) and brevity, all are designed to assess validated tobacco withdrawal symptoms and show substantial overlap with the DSM-V and ICD-10 criteria for nicotine withdrawal (Hughes, 2007b). Most of these measures appear to have adequate internal reliability, test-retest reliability, 
and content validity, and they all consider those commonly reported symptoms mentioned above (Hughes, 2007b; Hughes \& Hatsukami, 1986; Shiffman \& Jarvik, 1976). For example, use of these measures demonstrates that dependent cigarette smokers experience increases in symptoms such as difficulty concentrating, craving, irritability, and impatience with increasing periods of abstinence (Hughes \& Hatsukami, 1986; West, Ussher, Evans, \& Rashid, 2006; Welsch et al., 1999). Moreover, smokers' ratings of these symptoms decrease significantly following resumption of cigarette smoking (Etter \& Hughes, 2006; Blank, Disharoon, \& Eissenberg, 2009) or use of another nicotine/tobacco product (Molander, Lunell, \& Fagerström, 2000; Muramoto, Ranger-Moore, \& Leischow, 2003; Thornley et al., 2010).

While many of these withdrawal symptoms peak days after abstinence (Hughes, 2007a), they can be experienced within much shorter periods. For instance, ratings of withdrawal symptoms are reliably increased following a period of overnight abstinence ( $\geq 8$ hours), from not only cigarettes (Blank et al. 2009; Breland, Buchhalter, Evans, \& Eissenberg, 2002; Buchhalter \& Eissenberg, 2000), but also tobacco waterpipes (Maziak et al., 2009) and smokeless tobacco (Gray, Breland, Weaver, \& Eissenberg, 2008). Some evidence suggests that withdrawal symptoms may manifest even earlier, at least for cigarette smokers, with significant increases emerging between 30 and 180 minutes of abstinence (Hendricks et al., 2006).

Cognitive measures of withdrawal. Measures of performance on cognitive tasks are common objective measures of nicotine withdrawal (Ashare et al., 2014; Heishman et al., 1994; Hughes, 2007b, McClernon et al., 2015). Research on withdrawal-induced cognitive deficits tends to focus on three key cognitive domains of executive function (mental processes needed for attention and control of behavior; Diamond, 2013): sustained attention, working memory, and inhibitory control (Ashare et al., 2014; McClernon et al., 2015). 
Sustained attention. Sustained attention refers to the ability to maintain alertness to detect unpredictable signals over an extended length of time (McClernon et al., 2015). It is an executive function that allows individuals to discriminate between targets and distractors (Ashare et al., 2014; Sarter, Givens, \& Bruno, 2001), and appears to involve activation of the prefrontal and parietal regions of the right hemisphere (Kozink, Lutz, Rose, Froeliger, \& McClernon, 2010; Sarter et al., 2001). Examples of daily activities that involve sustained attention are reading, playing video games, and driving a vehicle. The inability to sustain attention while engaging in such activities may be reported as "difficulty concentrating" by smokers during a period of abstinence (Ashare et al., 2014; McClernon et al., 2015).

A number of studies have assessed the effect of nicotine/tobacco withdrawal on sustained attention using a variety of tasks, including the Rapid Visual Information Processing task (RVIP, Wesnes \& Warburton, 1984; McClernon et al., 2008; Hendricks et al., 2006), continuous performance tasks (McClernon et al., 2008; Harrison, Coppola, \& McKee, 2009), and vigilance tasks (Parrot, Garnham, Wesnes, \& Pincock, 1996; Hatsukami, Fletcher, Morgan, Keenan, \& Amble, 1989; Wesnes, Simpson, \& Kidd, 1988). All generally require participants to maintain vigilance to respond to targets appropriately over relatively long durations (McClernon et al., 2015). These tasks are relatively simple, in efforts to measure sustained attention per se, rather than aspects of other executive functions. For example, the RVIP task involves a series of single digits being presented on a screen at a rapid pace for an extended length of time, requiring participants to indicate when they notice three consecutive odd digits or three consecutive even digits (Wesnes \& Warburton, 1984).

Deficits in sustained attention have been demonstrated reliably for daily cigarette smokers that have abstained from nicotine/tobacco use. For example, McClernon et al. (2008) 
assessed the effects of overnight nicotine/tobacco withdrawal (9-11 hours) on multiple measures of sustained attention (e.g., RVIP, continuous performance task) in a sample of daily cigarette smokers. Smoking abstinence was associated with poorer accuracy on both tasks, though the continuous performance task revealed differences in speed (i.e., greater variability in response time for abstinent versus satiated conditions) that were not observed on the RVIP. This same pattern has been demonstrated consistently for abstinent and satiated cigarette smokers for the RVIP at different durations of abstinence (17 hours, Atzori, Lemmonds, Kotler, Durcan, \& Boyle, 2008; 10 hours, Mancuso, Andres, Ansseau, \& Tirelli, 1999; 12 hours, Leventhal, Waters, Moolchan, Heishman, \& Pickworth, 2010; Parrott \& Craig, 1992; “overnight”, Wesnes \& Warburton, 1984). Additionally, slowed reaction time for abstinent smokers on the RVIP may emerge between 30 to 240 minutes of abstinence (Hendricks et al., 2006). Convergent results have been observed using other measures of sustained attention (e.g., vigilance task, continuous performance task; Hatsukami et al., 1989; Parrott et al., 1996; Myers, Taylor, Moolchan, \& Heishman, 2008). Of course, some studies have shown a lack of effect of nicotine/tobacco withdrawal on measures of sustained attention (Cook, Gerkovich, Graham, Hoffman, \& Peterson, 2003; Kleykamp, Jennings, \& Eissenberg, 2011), but it is possible that cross-study differences in task, sample, and method of nicotine administration may account for different findings. Importantly, abstinence-induced decrements in sustained attention have been found repeatedly in the literature, even with these cross-study differences in task, method of nicotine administration, and duration of abstinence (see Evans \& Drobes, 2009 for review; Atzori et al. 2008; Foulds et al., 1996; Mancuso et al., 1999; Myers et al., 2008; Wesnes \& Warburton, 1984). Due to the overwhelming evidence demonstrating withdrawal-induced deficits in sustained 
attention, it is considered a key measure of cognition in nicotine withdrawal studies (Ashare et al., 2014; McClernon et al., 2015).

Working memory. Working memory refers to the concurrent storage and manipulation of information for a short period of time, with the prime function being coordination of resources (Baddeley, 1992; Baddeley \& Hitch, 1994). Working memory is essential for making sense of events that unfolds over time, such as solving math in your head or relating the beginning and end of a paragraph (Diamond, 2013). Additionally, working memory has an important role in reasoning and the performance of goal-directed behavior (Diamond, 2013; McClernon et al., 2015). The working memory system can be deconstructed into three subparts: central executive, phonological loop (verbal), and visuospatial sketchpad (nonverbal). The central executive includes an attentional controller and coordinates information from the other working memory components (Baddeley, 1992). The phonological loop can be further broken down into a phonological store (holds auditory information for up to two seconds) and an articulatory control process (maintains information and can register visual information into the phonological store). For example, the phonological store is important in speech perception and understanding, while the articulatory control process is involved in speech production and is used to rehearse and store verbal information. Together, these systems may be used to manipulate and rehearse verbal information, as in trying to remember a phone number. The visuospatial sketch pad performs similar functions, but is associated with visuospatial imagery as opposed to auditory information (Baddeley, 1992). It is useful for tasks such as spatial navigation and solving puzzles, and is important in the processing of shapes and colors of images. The prefrontal cortex is considered to be an essential brain area involved in working memory tasks (Knudsen, 2007), though association areas may differ depending on the aspect of working memory (e.g., verbal - language 
areas in temporal and inferior parietal cortex, Schumacher et al., 1996; visuospatial - inferior parietal cortex and high-order visual areas in occipital cortex, Smith, Jonides, \& Koeppe, 1996). Overall, working memory involves the attending to and processing of stimuli, and allows for rehearsal, retention, and manipulation of information.

One measure of working memory is the $n$-back task, which typically involves the presentation of a letter sequence and the requirement that participants indicate when the target stimulus does or does not match a stimulus presented " $n$ " letters back. For example, in a 2-back condition, participants must respond "yes" if the letter presented is the same as the letter presented two letters back (e.g., A - B - A), or "no" if the letter presented is not the same as the letter presented two letters back (e.g., A- B- C). In one study, a parametric assessment of the $n$ back task was used to compare working memory performance of abstinent ( $\geq 13$ hours) and satiated smokers (Mendrek et al., 2006). For this assessment, the researchers used conditions of different cognitive loads, including a 0-, 1-, 2-, and 3-back condition. Generally, abstinent smokers made more errors (saying "yes" to a non-target, saying "no" to a target) than satiated smokers, and had slower reaction times for the 2-back condition. The findings from this study may demonstrate that greater working memory impairment is observed at higher working memory loads (e.g., 0-back vs 2-back; McClernon et al., 2015; Mendrek et al., 2006).

Many studies demonstrate converging results of poorer $n$-back performance when smokers are in withdrawal (6 - 24 hours abstinence) as compared to satiated (Ernst, Hieshman, Spurgeon, \& London, 2001; Falcone et al., 2014; Grundey et al., 2015; Jacobsen et al., 2005; Kleykamp et al., 2011; Mendrek et al., 2006; Myers et al., 2008). Similar findings have also been observed with different measures of working memory (digit recall - Snyder, Davis, \& Henningfield, 1989). Similar working memory decrements are observed for abstinent cigarette 
smokers ( $\geq 12$ hours) when compared to nicotine administered in vehicles other than cigarettes, for instance nasal spray (Myers et al., 2008) and transdermal nicotine (Kleykamp et al., 2011). Like sustained attention, there are several studies that have reported no differences in working memory between abstinent and satiated smokers (Jacobsen, Pugh, Constable, Westerveld, \& Mencl, 2007; Wesnes, Edgar, Kezic, Salih, \& De Boer, 2013). However, there is greater evidence supporting withdrawal-induced working memory deficits, and studies demonstrating null results report potential issues with psychometric properties of the task (Wesnes et al., 2013).

Inhibitory control. Inhibitory control is an aspect of executive function that involves controlling one's attention, behavior, and thoughts to block out irrelevant stimuli while attending to appropriate information (Diamond, 2013). Inhibitory control also describes the ability to inhibit a dominant or automatic response (e.g., expecting to respond, but then needing to inhibit that response; McClernon et al., 2015). For example, a driver of a manual car may be unable to inhibit the automatic responses of shifting gears or using a clutch pedal when driving an automatic car. The main brain area associated with inhibitory control is the prefrontal cortex, specifically the right inferior frontal cortex (Kozink, Kollins, \& McClernon, 2010). Inhibitory control allows individuals to control impulses and remain focused on a task or goal. Additionally, it allows individuals to selectively attend to important stimuli, rather than immediately responding to all irrelevant stimuli in the environment (Diamond, 2013). For example, selective attention is needed at a cocktail party, when it is important to inhibit processing of the voices of guests except for the single individual with whom you are holding a conversation.

Tasks that measure inhibitory control typically involve participants responding to certain stimuli, and withholding responses to other stimuli (McClernon et al., 2015): Go/No-Go Task 
(Harrison et al., 2009; Kozink, Kollins et al., 2010; McClernon et al., 2008), Stop Signal Task (Ashare \& Hawk, 2012), and Stroop task (Atzori et al., 2008; Grundey et al., 2015; Zack, Belsito, Scher, Eissenberg \& Corrigall, 2001. For example, a Cued Go/No-Go task was used to assess smokers at differing levels of abstinence (Harrison et al., 2009). This task measures an individual's ability to respond when appropriate and withhold a response when no response is required. Specifically, participants are presented with a cue that indicates whether the upcoming trial will require a response or no response. Next, they receive a target indicating whether a response is required or not, and this target may match or be incongruent with the prior cue. Poorer performance (e.g., longer reaction times) was observed for daily cigarette smokers following 5-hours and 17-hours of nicotine/tobacco deprivation compared to a non-deprived condition (Harrison et al., 2009). Similar performance decrements have been reported in cigarette smokers that had been overnight abstinent (Hatsukami et al., 1989; Kozink, Kollins et al., 2010; McClernon et al., 2008). Additionally, poorer performance on inhibitory control tasks has been observed for abstinent smokers ( $\geq 6$ hours) using the Stop Signal task (Ashare \& Hawk, 2012) and the Stroop Task (Atzori et al., 2008; Grundey et al., 2015; Zack et al., 2001). Taken together, research consistently demonstrates that periods of nicotine/tobacco abstinence among cigarette smokers results in inhibitory control deficits.

Researchers have worked to use these same measurement tools to evaluate dependence and withdrawal related to ECIG use. Such work remains in its infancy for multiple reasons. First, ECIGs are a relatively newer tobacco product, having emerged into the U.S. market in 2007 (Hemmerich, Klein, \& Berman, 2017). Additionally, ECIG products have evolved at a rapid rate since their introduction (Brandon et al., 2015; Wagener et al., 2017). Over recent years, popular ECIGs have changed from cig-alikes to $2^{\text {nd }}$ generation tank models, to $3^{\text {rd }}$ generation mods that 
have high battery power and many modifiable components, and more recently to $4^{\text {th }}$ generation pods with high concentrations of nicotine salts. Thus, researchers are working to understand dependence and withdrawal associated with ECIG use while keeping up with advances in this relatively novel class of nicotine delivery products.

\section{ECIG Dependence and Withdrawal}

To date, there has been little research to evaluate nicotine dependence or withdrawal in exclusive ECIG users, though there has been one study to examine withdrawal in this sample over one week (Hughes et al., 2019b). Work that does exist confounds ECIG and cigarette use (Etter \& Eissenberg, 2015; Hughes et al., 2019a), as the large majority of ECIG users are current or former smokers of cigarettes (Schoenborn \& Gindi, 2015). For instance, a cross-sectional survey was administered to compare dependence on ECIGs relative to other tobacco products, though all of the ECIG users had a history of tobacco use: ECIG users who were current cigarette smokers, ECIG users who were former smokers, nicotine gum users who were former cigarette smokers, and current cigarette smokers who do not use ECIGs (Etter \& Eissenberg, 2015). Moreover, those ECIG users surveyed may have used ECIGs with or without nicotine. All respondents completed measures of nicotine/tobacco dependence in their original form (i.e., developed for cigarette dependence) or a form adapted for nicotine gum or ECIG products. Scores on these scales suggested that ECIGs are less dependence-producing than tobacco cigarettes and may be equally or less dependence-producing than nicotine gum (Etter \& Eissenberg, 2015). This idea is also supported by other work that includes current ECIG users who formerly used cigarettes; dependence scores were shown to be lower, time to first product use in the morning was shown to be longer (i.e., the duration of product abstinence was longer), and ratings of nicotine/tobacco withdrawal and craving were shown to be lower for ECIGs 
relative to cigarettes (Farsalinos, Romagna, Tsiapras, Kyrzopoulos, \& Voudris, 2013b; Foulds et al., 2015). Only one available study has suggested that ECIGs may produce higher levels of dependence than cigarettes (Johnson et al., 2018), though the large majority of the sample reported previous cigarette use $(86.2 \%)$. The best evidence of dependence on ECIGs, and the phenomenon of ECIG withdrawal, requires evaluation of ECIG users with no history of cigarette or other tobacco use.

Several product and ECIG use characteristics may be predictive of ECIG dependence. Use characteristics such as longer durations of ECIG use (Foulds et al., 2015; Johnson et al., 2018), as well as daily ECIG use (Morean, Kirshnan-Sarin, \& O’Malley, 2018), are associated with increased ECIG dependence. Additionally, frequency of ECIG use increased in an adolescent sample over 12 months along with increased dependence, which may be suggest that users are gaining tolerance (Vogel, Prochaska, Ramo, Andres, \& Rubinstein, 2019). Liquid consumption is strongly correlated with the number of puffs taken and puff duration (Farsalinos et al., 2013a), and puff duration is associated with ECIG nicotine delivery (Blank et al., in press). However, others have found that vaping consumption variables may not be associated with ECIG dependence (Browne \& Todd, 2018). Regarding product characteristics, using more advanced generations of ECIGs is associated with higher ECIG dependence (Foulds et al., 2015; Yingst et al., 2015). This finding may be a result of increased speed and concentration of nicotine delivery associated with newer generations of ECIGs (Wagener et al., 2017). Relatedly, higher nicotine concentrations (Hiler et al., 2017; Ramôa et al., 2016) and increased wattages (Kosmider, Spindle, Gawron, Sobczak, \& Goniewicz, 2018; Talih et al., 2015) can increase nicotine yield and delivery, which may lead to increased ECIG dependence. Indeed, higher nicotine concentrations are associated with increased ECIG dependence (Foulds et al., 2015) and 
increasing nicotine concentrations may be evidence of the development of tolerance (Vogel et al. 2019). However, the interactive relationships between some of these variables (e.g., nicotine concentration, wattage, device type, liquid consumption) may complicate their associations with nicotine delivery and dependence (Shihadeh \& Eissenberg, 2015). For example, studies that demonstrate the relationship between nicotine concentration and nicotine delivery hold other ECIG specifications constant (Hiler et al., 2017; Ramôa et al., 2016), but this is likely not ecologically valid. Indeed, individuals that use lower nicotine concentrations tend to vape at higher wattages and consume more liquid than individuals using higher nicotine concentrations (Smets, Baeyens, Chaumont, Adriaens, \& Van Gucht, 2019). Though higher-powered devices may deliver nicotine more quickly, users of higher and lower powered devices have demonstrated similar plasma nicotine concentrations after a 2-hour ad lib vaping session (Wagener et al., 2017), indicating that users may vape differently to compensate for differences in ECIG specifications. Ultimately, more work must be done to understand how use characteristics and ECIG specifications interact to influence nicotine delivery and how these variables may promote dependence in ECIG users.

Indeed, researchers have argued that nicotine dependence is product-specific, and should be assessed accordingly (Fagerström \& Eissenberg, 2012). One reason for this argument is that products that deliver nicotine do so with different speeds of delivery to the brain (Henningfield \& Keenan, 1993; West et al., 2000). Products that deliver nicotine more rapidly (e.g., cigarettes) are more likely to support dependence than those that deliver nicotine at a slower speed (e.g., nicotine patch; Le Houezec, 2003). As for ECIGs, Foulds et al., (2015) noted that these devices generally deliver nicotine more slowly and in lower concentrations compared to cigarette smoking. However, more recent generations of ECIGs have demonstrated an increased speed and 
concentration of nicotine delivery (Wagener et al., 2017), which may lead to greater nicotine dependence in users of these devices compared to users of the original models. Evidence in support of this idea derives from the observation that dependence scores are higher among users of newer generations of ECIGs than users of "cig-alike" style ECIGs (Yingst et al., 2015). Another reason for this argument of product-specific dependence assessment is that nicotine may be a stronger reinforcer in the presence of certain non-nicotine stimuli (Perkins, Karelitz, \& Boldry, 2017; Rupprecht et al., 2015). For example, nicotine may enhance the reinforcing efficacy of certain cues associated with nicotine/tobacco product use (Fagerström \& Eissenberg, 2012). While some cues may overlap between tobacco cigarettes and ECIGs (e.g., hand-tomouth movements, cloud of vapor/smoke, size/shape for some ECIG models), ECIGs are a different class of products that likely have distinct cues separate from traditional cigarettes (e.g., taste/aroma, size/shape for some ECIG models). As ECIGs continue to evolve, it is possible that cues shared with cigarettes will become less common and thus ECIG-exclusive cues will need to be considered for evaluation. This idea is evidenced by the change in ECIG design from cig-alike (e.g., LED light that resembles burning end of a cigarette, similar size/shape to cigarettes) to modern models (e.g., larger size, heavier weight, some require manual activation prior to each puff).

Of course, the evaluation of ECIG dependence and withdrawal among users of a specific ECIG device/liquid combination is a challenge unlikely to be overcome in the current market. As of 2014, there were at least 460 different ECIG brands and at least 7,764 different liquid flavors available for purchase (Zhu et al., 2014). Additionally, the regulation of ECIGs will not be in full effect until 2022 (U.S. Food and Drug Administration, 2017), thus allowing manufacturers the 
freedom to make design changes until that time. Still, work is needed on the evaluation of ECIG dependence and withdrawal in ECIG users without a history of cigarette use.

\section{Statement of the Problem}

Nicotine/tobacco dependence is a problem that negatively affects health at an individual and population level. Currently, the tolerance and withdrawal experienced by cigarette smokers is well-established. Specifically, deficits in concentration, attention, and memory are commonly reported withdrawal symptoms that are objectively observed using cognitive tasks. Subjective measures indicate many symptoms that abstinent smokers report, such as cravings, urges, difficulty concentrating, anxiousness, among others. While withdrawal in cigarette smokers is well-categorized, less is known about dependence and withdrawal associated with newer products, such as ECIGs. Cigarette-like nicotine delivery has been reported for some ECIGs, indicating that they also may be dependence causing. However, extent work that addresses the ECIG dependence and withdrawal confounds ECIG and cigarette use. Thus, the purpose of this project is to characterize the cognitive, subjective, and physiological measures of ECIG withdrawal during a period of abstinence in ECIG users with little history of cigarette smoking.

\section{Clinical and Regulatory Implications}

Characterizing ECIG dependence and withdrawal is meaningful for clinicians and tobacco regulators. Clinicians should evaluate ECIG use and dependence with similar methods as used for cigarettes. Additionally, development of treatment efforts for ECIG cessation may be necessary. To date, no studies have been published that assess the best methods to help ECIG users quit. Knowledge of the timecourse and primary symptoms of withdrawal will aid clinicians and regulators in determining the optimal treatments for ECIG cessation. Additional concerns arise when considering cigarette smokers switching to ECIG use, which may not result in a 
significant reduction of nicotine dependence. A similar issue is dual use of cigarettes and ECIGs, because these users are likely to strengthen their dependence on nicotine if ECIGs are dependence-causing. The long-term health effects of ECIG use remain unknown, and efforts should be targeted towards complete nicotine cessation or use of FDA-approved nicotine replacement therapies for all users.

Additionally, the Family Smoking Prevention and Tobacco Control Act of 2009 declares that modified risk tobacco products must benefit the population as a whole considering both people who do and do not currently use tobacco products. Thus, regulators should be concerned if individuals who would otherwise not use tobacco become dependent upon ECIGs. This issue is particularly troublesome given high rates of ECIG use among adolescents and young adults (Audrain-McGovern, Stone, Barrington-Trimis, Unger, \& Leventhal, 2019; Leavens, Stevens, Brett, \& Leffingwell, 2019), which is a period particularly sensitive to development of nicotine dependence (Lanza \& Vasilenko, 2015). The potential for otherwise non-tobacco using individuals to become dependent upon ECIGs may influence the consideration of modified risk status, marketing regulations, and warning labels placed upon ECIG companies. Similarly, regulators may need to develop information campaigns to ensure individuals, particularly youth, are aware of the possibility and implications of nicotine dependence.

\section{Chapter 2 - Method}

\section{Selection of Participants}

Participants were recruited through word-of-mouth and university-approved advertisements around the greater Morgantown area. Additionally, participants were recruited through internet websites such as Facebook and Craigslist. A total sample size of 10 experienced ECIG users was sufficient to detect effects for primary outcomes of subjective ratings (e.g., 
craving, urge) and heart rate assuming a moderate to large effect size (Cohen's $f>0.25$, Cohen, 1988), a large correlation between repeated measures $(r=0.80)$, a desired power of 0.80 , and a Type I error rate of 0.05 .

Inclusion criteria. The ECIG-experienced participants recruited for this study must have self-reported a) use of a nicotine-containing ECIG for $\geq 6$ months, b) use $\leq 10$ cigarettes in the past year and c) use $\leq 2$ cigarettes in the past month. Current ECIG use was confirmed via a semi-quantitative urinary cotinine value $\geq 3$ (NicAlert ${ }^{\circ}$; Nymox Pharmaceutical Corporation, Quebec, Canada), while current non-smoking status was confirmed via an exhaled air carbon monoxide level < 5 ppm (Perkins, Karelitz, \& Jao, 2013; Hiler et al., 2017). ECIGs are not a combustible product and do not generate carbon monoxide; therefore, the carbon monoxide level of ECIG users who do not smoke cigarettes should be akin to that of a non-smoker. Still, participants were required to use a nicotine containing ECIG, and thus cotinine levels, a metabolite of nicotine, should indicate nicotine use. No parameters were placed on other ECIG use characteristics due to the changing landscape of the ECIG market. While previous work required that participants use a specific amount of ECIG liquid daily at a specific concentration of nicotine (e.g., $1 \mathrm{ml} /$ day of $12 \mathrm{mg} / \mathrm{ml}$ nicotine; Spindle et al., 2017), these criteria may no longer be viable. For instance, the popularity of "pod mod" systems have risen (Huang et al. 2019), and such systems typically have a much higher nicotine concentration which may lead to a lower volume of liquid consumed per day relative to older devices. Juul pod mods, which account for over $40 \%$ of the ECIG market share (Huang et al., 2019), contain nicotine doses as high as 69-75 ml/mg (Eissenberg et al., 2018; Talih et al., 2019) and deliver pharmacologically active doses of nicotine in regular cigarette smokers (e.g., $\sim 11 \mathrm{ng} / \mathrm{ml}$ plasma level) (Eissenberg et al., 2018). Recent work also reveals relatively high levels of nicotine in the urine of 
adolescents aged 13-21 years who report current use of a pod mod system such as Juul (Goniewicz, Boykan, Messina, Eliscu, \& Tolentino, 2018). Together with other work (Wagener et al 2017), users of a wide range of ECIG doses in combination with many different types of ECIGs may be consuming levels of nicotine that promote dependence. To maximize the likelihood of enrolling those who are dependent, participants also must have scored $\geq 7$ on the Penn State Electronic Cigarette Dependence Index (Foulds et al., 2015). From previous work with ECIG experienced users (Felicione et al., 2019) the median score on this index was 6 (range $=1-15)$. While this score is considered low - moderate (range $=0-20$; Foulds et al., 2015), the reliability and validity of this scale has yet to be proven. Still, use of this cutoff ensures that we excluded ECIG users with the lowest levels of dependence. Finally, participants must have selfreported an age of between 18 to 40 years, which should reduce the variability associated with age-related changes in withdrawal effects and cognition (Braver et al., 2001; Falcone et al., 2014).

Exclusion criteria. Individuals were excluded if they reported an uncontrolled medical condition, a diagnosed psychiatric condition such as schizophrenia or bipolar disorder, regular use of most prescription medications (e.g., excluding vitamins and birth control), marijuana use $>10$ days in the past 30 days, alcohol use $>25$ days in the past 30 days, and use of other illicit substances in the past month. Women who reported breastfeeding or pregnancy, or tested positive for pregnancy via urinalysis, were excluded. These exclusionary criteria have been used successfully in other research with ECIG-experienced users (Ramôa et al., 2016; Spindle et al., 2017).

Telephone screening procedure. Individuals who responded to advertisements were screened initially via telephone interview or an online screening questionnaire. Laboratory staff 
read an approved transcript to explain the study procedures and requirements to interested callers. Interested individuals were asked to report on basic demographic, medical history, and drug use information (Appendix A). Individuals who appeared eligible based on this telephone or online screening were asked to visit the lab for an in-person screening (as in Blank et al., 2009; Felicione et al., 2019; Kleykamp et al., 2011; Spindle et al., 2017). Callers were told that the purpose of the study was to assess the short-term effects of ECIGs in order to better mask the purpose of the study to avoid participant expectation effects.

Informed consent \& in-person screening procedures. Individuals were guided through the informed consent form to demonstrate study purpose, study procedures, potential risks and benefits of participation, and payment. Those who were willing and able to consent completed additional screening procedures. The laboratory screening visit consisted of questionnaires similar to those administered via the telephone interview (Appendix B-C). The purpose of these near-identical screening questionnaires was to ensure reliability of reporting, and individuals whose answers were conflicting were considered ineligible for participation. A trained staff member collected a urine sample from women to test for pregnancy (QuickVue, Quidel Corporation, San Diego, CA), as well as a urine sample from all potential participants to test for cotinine. Staff members also administered the Mini International Neuropsychiatric Interview (MINI) for Tobacco Use Disorder (Sheehan et al., 1998; Appendix D), which served as a clinical tool to determine the severity of tobacco dependence of participants. The MINI is a short diagnostic interview developed by clinicians and psychiatrists and associated with the DSM to diagnose tobacco use disorder, as well as other psychiatric disorders. Individuals who agreed to join the study and met the eligibility criteria for participation participated in a training session, and then scheduled their first study session. 


\section{Study Design}

The study used a within-subjects design with two randomly-ordered sessions that differed by the duration of nicotine/tobacco abstinence: 0 ( $a \mathrm{~d}$ lib condition) or $180 \mathrm{~min}$ (abstinent condition). Participants were assigned to these two conditions using matched random assignment based on their percent correct for the RVIP task at baseline. Each session was approximately 3.5 hours in length, and sessions were separated by a minimum of 48 hours (Kleykamp et al., 2011). Within each session, participants responded to a cognitive battery at two different time points, as well as subjective questionnaires every $30 \mathrm{~min}$.

Training session. Participants practiced each cognitive task in a training session to minimize potential effects of practice (Mendrek et al., 2006). Specifically, participants completed an approximately two-hour practice session at the in-person screening visit, after they were determined to be eligible for this study. In this visit, the tasks were explained and demonstrated for participants, and participants completed practice versions of all three cognitive measures. Participants completed all cognitive tasks at least once, but no more than four times, during this training session. Training for a task was complete when the participant either: a) achieved greater than $70 \%$ accuracy, b) achieved consistent scores on repeated administration of a task, or c) achieved poorer performance with repeated administrations of a task. Previous reports suggest that three 30-min practice sessions can eliminate practice effects completely (Wesnes \& Warburton, 1984), and other studies have condensed training into one, longer training session (McClernon et al., 2008). Participants were paid $\$ 10$ upon completion of the training session.

Session procedure. All session procedures described below are outlined in Figure 1. As shown in this figure, both laboratory sessions began and ended at a consistent time and lasted 
approximately 3.5 hours. Sessions began by collecting a breath sample to verify that $\mathrm{CO} \leq 5$

ppm. Continuous monitoring of heart rate commenced at the start of a session. The first $30 \mathrm{~min}$ of the session served as a habituation period to gather baseline physiological data and allow participants to become accustomed to the laboratory. Next, participants began a 5-min bout of ECIG puffing to standardize the time since last ECIG use. During this puffing bout, a research assistant instructed participants to take 10 puffs with a 30 -sec interpuff interval (Blank et al., 2009; Felicione et al., 2019). Participants then completed subjective questionnaires that assessed nicotine/tobacco withdrawal symptoms followed by a battery of cognitive tasks. Completion of this cognitive battery took approximately $20 \mathrm{~min}$. In the abstinent condition, participants were not permitted to use an ECIG following the initial puffing bout. In the ad lib condition, participants were permitted to use their own ECIG ad lib throughout the session, with the exclusion of using the ECIG during the cognitive battery. Sessions were videotaped to characterize puff topography during the ad lib condition.

Thirty min following the completion of the initial puffing bout, subjective questionnaires were administered again. These same subjective questionnaires were measured every 30 min for 180 min, with a total of 7 measurements. The cognitive battery was administered at one additional time during this same period, at $120 \mathrm{~min}$. One hundred and twenty min was chosen as an assessment timepoint based on previous research demonstrating withdrawal-induced cognitive deficits within two hours of smoking abstinence (Hendricks et al., 2006; Parrott et al., 1996). Participants were permitted to eat lunch during the session, though all food and beverages were required to be caffeine-free. Caffeine may improve performance on cognitive measures such as sustained attention/vigilance tasks (for review, see Smith, 2002) and thus, may confound results if consumed during sessions. Throughout the session, participants were allowed to engage in 
activities with a low cognitive load (e.g., watch movies, play games of phone, read book), but were not permitted to engage in tasks that involve a high cognitive load (e.g., writing an essay), Participants were paid 60 dollars at the completion of each session (plus 10 dollars at training), for a total amount earned of 130 dollars.

\section{Materials}

ECIG devices. Participants were required to use their own ECIG liquid and device combination, as opposed to standardizing these features (as in Spindle et al., 2017). The ECIG market is currently inundated with innumerable combinations of liquid flavors, solvents, and nicotine concentrations, as well as battery voltage and atomizer resistance levels. Thus, not only it would be nearly impossible to choose a single best combination of these features, but generalizability would also be limited. Moreover, our previous work demonstrates that use of a standardized ECIG liquid/device by experienced ECIG users may prevent product consumption during the ad lib condition. That is, some participants who found the standardized product to be less than satisfactory, based on spontaneous verbal reports and/or ratings on questionnaires related to sensory cues or withdrawal suppression, failed to engage in ECIG use during one or more ad libitum puffing bouts. Importantly, a small sample of the liquid used by participants was collected for later analysis to confirm that the nicotine concentration is at least $3 \mathrm{mg} / \mathrm{ml}$ nicotine.

Computer equipment. All cognitive tasks were administered via the E-Prime 3.0 computer software (Kleykamp, Jennings, Blank, \& Eissenberg, 2005; Schneider, Eschman, \& Zuccolotto, 2002). Subjective questionnaires were administered via computerized software (Clinical Research Support Systems). Physiological data was recorded through the same software as subjective questionnaires. 
Video equipment. A Canon Vixia HF R42 (Canon USA, Inc.; Melville, NY) was used to record ECIG use for ad lib conditions. This video camera has been used successfully to record ECIG puffing bouts in previous research (Felicione et al., 2019). The video data was imported to and analyzed in Adobe Premier Pro 2015 (Adobe Systems, Inc.; San Jose, CA).

\section{Primary Outcome Measures}

Primary outcome measures were heart rate and subjective ratings of withdrawal and nicotine effects. An additional primary outcome included dependence characteristics of individuals that completed a telephone screening.

Heart rate. Heart rate was measured continuously throughout each session (Noninvasive Patient Monitor model 506 NP3, Criticare Systems, Inc., Waukesha, WI). Heart rate was captured via finger pulse oximeter that can capture a range of 20 to 300 beats per min with an update time of one sec and an accuracy of $+/$ - one beat per min or one percent of the reading (Criticare Systems, Inc.). Heart rate was collected every $20 \mathrm{sec}$ and then transferred to a computer. Heart rate data were averaged into 5-min bins to create single values at each 30-min timepoint. Measurement of heart rate served multiple purposes. First, changes in heart rate are indicative of exposure to nicotine (Hendricks et al., 2006; Jolma, Samson, Klewer, Donnerstein, \& Goldberg, 2002; Omvik, 1996). Second, heart rate was used to monitor participant safety, and a medical monitor was contacted if a participant's vital signs fall out of a predetermined ranged (see subsection Participant Safety and Rights). These materials and methods for collecting physiological data have been used to maintain data integrity and participant safety in both cigarette smokers (Blank et al., 2009; Breland et al., 2002; Cobb, Shihadeh, Weaver, \& Eissenberg, 2011) and ECIG users (Felicione et al., 2019; Spindle et al., 2017). 
Minnesota nicotine withdrawal scale. The Minnesota Nicotine Withdrawal Scale (MNWS; Hughes \& Hatsukami, 1986) questionnaire (Appendix E) is a measure of nicotine and tobacco withdrawal symptoms. The questionnaire contains 11 items: "Urges to smoke," "Irritability/frustration/anger," “Anxious," "Difficulty concentrating," "Restlessness," "Hunger," "Impatient," "Craving a cigarette/nicotine," "Drowsiness," "Depression/feeling blue," and "Desire for sweets". Items specifically related to cigarettes ("Craving a cigarette/nicotine") were adapted to electronic cigarettes. Participants were shown the word or phrase centered above a horizontal line serving as a visual analog scale. The left end of the line showed the phrase "not at all" $($ score $=0)$, and the right end of the line showed the phrase "extremely" $($ score $=100)$. Participants moved the cursor to any point of the line and click to create a vertical mark, which could be moved before the participants continues to the next question. Each score was expressed as a percentage of the total line length from the left end to the vertical mark. Internal consistency for this measure has not been assessed, however, the items are known to be valid and reliable indicators of withdrawal symptoms (Hughes \& Hatsukami, 1986; Hughes, 2007b). This questionnaire has been commonly used to assess withdrawal symptoms in cigarette smokers (Blank et al., 2009; Breland, Kleykamp, \& Eissenberg, 2006; Bullen et al., 2010; Cobb et al., 2011; Wagener et al., 2014) and more recently, in ECIG users (Felicione et al., 2019; Spindle et al., 2017; St. Helen et al., 2016)

Tiffany-Drobes questionnaire of smoking urges: brief form (QSU-Brief). The QSUBrief (Appendix F, Cox, Tiffany, \& Christen, 2001; Tiffany \& Drobes, 1991) consists of 10 Likert-scale items measuring multidimensional features of nicotine and tobacco cravings. Statements (e.g., "All I want right now is a cigarette"; "I am going to smoke as soon as possible") were presented above seven boxes, with the leftmost labeled "strongly disagree" 
$($ score $=0)$ and the rightmost labeled "strongly agree" (score $=6)$. Participants moved the cursor to select one box, which could be adjusted before continuing to the next item. Like the MNWS, statements were adapted by replacing "cigarette" and "smoke" with "ECIG" and "vape", respectively. Items were collapsed into two factors previously defined by factor analysis: intention to smoke (scale range $=0-30$ ) and anticipation of relief from withdrawal (scale range $=$ 0-24). The QSU-Brief has demonstrated high reliability (Cronbach's alpha $=0.97$, Cox et al., 2001) and internal consistency within each of the two factors (Cronbach's alpha $=0.96,0.92$; Cox et al., 2001). The QSU and QSU-Brief are common measures of nicotine and withdrawal symptoms in cigarette smokers (Blank et al., 2009; Breland et al., 2006; Cobb et al., 2011; Norton, June, \& O’Connor, 2014) and ECIG users (Felicione et al., 2019; St. Helen et al., 2016; Vansickel \& Eissenberg, 2013).

Direct effects of nicotine scale (DENS). The DENS (Appendix G; Evans, Blank, Sams, Weaver, \& Eissenberg) is a 10-item VAS that assesses the intensity of nicotine-associated side effects. The questionnaire items include "nauseous," "dizzy," "lightheaded," "nervous," "sweaty," "headache," “excessive salivation," "heart pounding," "confused," and "weak." This questionnaire has been used to assess nicotine effects associated with ECIG use (Felicione et al., 2019; Hiler et al., 2017; Spindle et al., 2017).

Direct effects of product scale (DEPS). The DEPS (Appendix H) is a 9-items VAS to assess effects commonly associated with cigarette smoking, but were modified to ask about vaping. Questions began with the prompt "was the product..." or "did the product..." followed by "satisfying," "pleasant," "taste good," "make you dizzy," "calm you down," "help you concentrate," "make you feel more awake," "reduce your hunger for food," and "make you 
sick?" Similar to the DENS, this questionnaire has been used to assess effects of vaping after bouts of ECIG use (Felicione et al., 2019; Hiler et al., 2017; Spindle et al., 2017).

ECIG dependence and use. All individuals that completed a telephone/online screening were asked questions about their cigarette use history (lifetime, past year, past month), as well as ECIG use history and ECIG dependence (see specific items in Appendix A). Specifically, screening questionnaires included information about a) ECIG device and liquid specifications (e.g., model, liquid nicotine concentration, flavor, wattage), b) amount of liquid used (ml/day), c) frequency (days/week) and duration (months/years) of ECIG use, and d) ECIG dependence (Penn State Electronic Cigarette Dependence Index). Regarding enrolled participants, study staff observed participants' ECIG devices and liquid to verify the specifications reported by the participant at screening.

\section{Secondary Outcome Measures}

Secondary outcomes included those related to cognitive processing - attention, working memory, and inhibitory control - and also the reinforcing efficacy of ECIGs (choice). Additional secondary outcomes included characterizing puff topography in the ad lib condition.

Rapid visual information processing task (RVIP). The RVIP is one of the most frequently used tasks of sustained attention among smokers (Wesnes \& Warburton, 1983). This task involves a series of single digits being presented on a computer screen at a rapid pace. Participants were instructed to press a response key when they noticed three consecutive odd digits or three consecutive even digits. This task has been used extensively to measure abstinence-induced deficits in attention in cigarette smokers (Gilbert et al., 2005; Hendricks et al., 2006; McClernon et al., 2008; Wesnes \& Warburton, 1983; Wesnes \& Warburton, 1984). Specifically, research has demonstrated that smokers may show accuracy reduction and slowed 
reaction time for sustained attention tasks at 2 hours of deprivation, with peak effects at 6 hours (Parrott et al., 1996).

As depicted in Figure 2, digits were presented in the center of a computer screen at a rate of 100 digits per min (Gilbert et al., 2005; Hendricks et al., 2006) for approximately 6 min. Previous research has included a 9-min version of this task (Gilbert et al., 2005; McClernon et al., 2008), though differences between abstinent and non-abstinent smokers have been detected within the first 4 min (Herbert, Foulds, \& Fife-Schaw, 2001). Response targets were set to appear eight times per min, with 5 to 30 digits appearing between each target (Hendricks et al., 2006). The window in which participants must respond lasted 1500 milliseconds (ms), though digits continued to appear at a constant rate (Hendricks et al., 2006; Herbert et al., 2001), and the response was the press of a computer key. Specifically, participants were asked to press the key after each sequence of three consecutive odd or even digits (e.g., 7-3-1, or 2-8-6).

The RVIP task yielded four dependent variables. Correctly identified targets (measured via "percent correct") were defined as a press on the response key when three consecutive odd or even digits are presented. Reaction time to targets (measured in ms) was defined as the latency to press the response key when three consecutive odd or even digits are presented. Errors of commission (measured via number of incorrect responses) were defined as a press on the response key when there was not three consecutive odd or even digits presented ("false positive"). Finally, errors of omission (measured via number of missed targets) were defined as a lack of a press on the response key when three consecutive odd or even digits are presented (“false negative"; Hendricks et al., 2006).

$N$-back task. The $n$-back task (Jonides et al., 1997) served as a cognitive assessment of working memory. The 2-back and 3-back version of this task was used (Kleykamp et al., 2005; 
Kleykamp et al., 2011), as previous research has revealed no differences between satiated and abstinent smokers for 0-back and 1-back versions (Mendrek et al., 2006). The $n$-back task involved a block of trials with a 45-letter sequence presented to subjects. Each letter was presented in the center of a blank screen for $500 \mathrm{~ms}$, with a 2.5 -sec interstimulus interval (Jonides et al., 1997; Falcone et al., 2014; Myers et al., 2008). The 45-letter sequence was designed so that there are 14 letters that correctly match the $n$-back target. Each time the $n$-back task was measured, participants responded to one block for the 2-back condition and one block for the 3-back condition. Completion of the $n$-back task took approximately $4.5 \mathrm{~min}$.

For the 2-back condition (see Figure 3, Panel A), participants first saw the instruction "Find 2-back." They were required to press the "yes" key when the letter presented was identical to the letter displayed two letters prior (e.g. A-B-A), and not respond when the letter presented was different from the letter displayed two letters prior (e.g., A-B-C). Procedures for the 3-back condition mimicked the 2-back condition, except the 3-back condition involved identifying the target letter that is displayed 3 letters prior (e.g., A-B-C-A) (see Figure 3, Panel B). The $n$-back task has been used readily in assessing cognitive performance of cigarette smokers (Mendrek et al., 2006; Kleykamp et al., 2011)

There were two dependent variables associated with the $n$-back task: accuracy and reaction time to correct "yes" responses. Accuracy was defined as the proportion of hits minus the proportion of false positives. A hit is a correctly identified $n$-back target (e.g., A-B-A for 2back, A-B-C-A for 3-back) and a false positive is a response that does not match the $n$-back condition. Reaction time to correct "yes" responses (measured in ms) is defined as the latency to respond when the participant correctly identifies an $n$-back target. These variables will be analyzed separately for the different $n$-back conditions. 
Cued go/no-go task. The Cued Go/No-Go task is a behavioral measure of inhibitory control, testing a participant's ability to respond when appropriate and to withhold a response when no response is required (McClernon et al., 2015). The Go/No-Go task for the proposed study was based on a method used to assess effects of caffeine and alcohol (Marczinski \& Fillmore, 2003), as well as smoking abstinence (Harrison et al., 2009; McClernon et al., 2008).

Figure 4 depicts the Cued Go/No-Go task. Each trial began with the presentation of a cross in the center of the screen on which participants were asked to fixate their attention. This "fixation cross" was presented for $800 \mathrm{~ms}$ followed by a blank screen for $500 \mathrm{~ms}$. After the blank screen, a cue was presented in the center of the screen for 100, 200, 300, 400, or $500 \mathrm{~ms}$. The cue, a white rectangle with a black outline, alternated from being in a vertical or horizontal orientation for each of $50 \%$ of trials. A vertically-presented rectangle was indicative of an upcoming "go" target, while a horizontally-presented rectangle was indicative of an upcoming "no-go" target. After the cue was presented for the pre-defined length of time, it changed from white to either blue or green (for printing purposes, blue is represented as gray and green is represented as black in Figure 4). In trials when the rectangle became green, the participant must press the response key as quickly as possible (go trial). In trials when the rectangle became blue, the participant must withhold a response (no-go trial). The cue appeared on the screen for 1000 ms, or until the participant made a response. Cues may have been correct or incorrect. For example, on $80 \%$ of trials, the orientation of the rectangular cue correctly informed the participant about the type or target that will follow (i.e., vertical and green, horizontal and blue). For $20 \%$ of the trials, the cue and the target were incongruent (i.e., horizontal and green, vertical and blue). A total of 250 trials were presented over 5 blocks. The variable and random duration 
of the cue, along with the $80 \%$ probability of a congruent cue and target, may have prevented participants from anticipating the upcoming cue (Marczinksi \& Filmore, 2003).

There were three dependent variables associated with the cued Go/No-Go task: response inhibition for correctly cued trials, response inhibition for incorrectly cued trials, and response execution. Response inhibition (measured via "percent correct") was defined as the proportion of correct non-responses to no-go trials. Response inhibition was calculated separately for correctly cued trials (No-Go accuracy when the rectangle orientation and color change are consistent) and incorrectly cued trials (No-Go accuracy when the rectangle orientation and color change are inconsistent). Response execution (measured in ms) was defined as the latency to respond on go trials (Marczinski \& Fillmore, 2003; McClernon et al. 2008).

Multiple choice procedure. The Multiple Choice Procedure (MCP; Appendix I) is a validated method to assess the abuse liability of a drug for a human in a laboratory setting (Grffiths, Triosi II, Silverman, \& Mumford, 1993; Griffiths, Rush, \& Puhala, 1996). This procedure involves a participant making choices of a preference for a drug or increasing amounts of money. Specifically, this task included 10 trials in which participants chose between a fixed level of ECIG use (10 ECIG puffs) or increasing amounts of money $(\$ 0.01,0.02,0.04,0.08$, $0.16,0.32,0.64,1.28,2.56,5.12)$. The dependent variable for this task was the crossover point, or the trial at which a participant switched from choosing 10 puffs to an amount of money. This procedure has been used to assess the abuse liability of a variety of drugs, including ECIGs (McPherson et al., 2016; Vansickel, Weaver, \& Eissenberg, 2012).

Puff topography. Puff topography served as the primary measure of ECIG consumption. Puff topography variables that can be measured via video include puff number (the amount of puffs), puff duration (the length of a puff, measured in sec), and interpuff interval (IPI, the time 
between puffs, measured in sec). Ad lib sessions were videotaped and scored by two independent raters to determine puff number, puff duration, and IPI throughout the session. The operational definitions used for these variables are based on our previous work that evaluated the reliability and validity of measurement methods for ECIG puff topography (Felicione et al., 2019). Puff duration was defined as the amount of time between the first frame in which a participant's lips are enclosed around the ECIG until the last frame in which their lips remain enclosed around the ECIG. IPI was defined as the amount of time between the first frame in which a participant's lips are removed from the ECIG (e.g., first frame after a puff ends) until the last frame before the next puff starts. Puff number was defined as the number of puffs that are $\geq 300 \mathrm{~ms}$ with an IPI $\geq$ $300 \mathrm{~ms}$. Puffs with $<300 \mathrm{~ms}$ IPIs were collapsed into one puff, and any remaining puffs $<300$ ms durations were deleted (similar to Felicione et al., 2019; Hiler et al., 2017; Spindle et al., 2017)

Currently, there is no accepted definition of a single ECIG use bout (for review, see Blank et al., 2016). Recent work (St. Helen et al., 2016), however, has attempted to characterize such bouts using "puff clusters," or the number of puffs with no more than $60 \mathrm{sec}$ between each puff ( $\leq 60 \mathrm{sec}$ IPI). These clusters can be further categorized by the number of puffs in a cluster: short ( $2-5$ puffs), medium (6 - 10 puffs) and long ( $>10$ puffs). We modeled this approach using topography data from the ad lib session. All video-based topography variables were scored by two independent raters and compared for assessment of interrater reliability.

\section{Participant Safety and Rights}

Participant's safety and rights were assured through an IRB-approved protocol enacted by trained laboratory staff. Participants were made aware of the Office of Research Integrity and Compliance and the fact that they can contact this office with questions about their rights as a 
participant. Participants were not expected to incur adverse events, given that they are experienced ECIG users and will be using their own ECIG to puff throughout the session.

Participants were warned that they may experience aversive withdrawal symptoms from tobacco abstinence such as irritability, anxiety, and restlessness (Hughes \& Hatsukami, 1986; Rubinstein, Benowitz, Auerback, \& Moscicki, 2009). These withdrawal effects may cause discomfort; however, they are not medically dangerous. To ensure safety throughout the session, physiological measure of heart rate and blood pressure were monitored continuously. A medical monitor would have been contacted regarding participant safety if heart rate exceeds the range of 50 to $110 \mathrm{bpm}$, though this issue never occurred. Confidentiality was assured by using coded identity numbers for participant data and storing data in locked room and on password protected computers.

\section{Data Preparation and Analysis}

Data preparation. Data for the cognitive tasks and ECIG puff topography was prepared based on the dependent variables described above. Heart rate data was averaged into 5-min bins to create single values at each 30 -min time point, for a total of 8 values.

Feasibility and recruitment. Feasibility of the method was explored by examining enrollment and attrition rates. This descriptive analysis included assessing the number of participants screened, enrolled, and completing the study. Also reported were reasons for ineligibility and baseline characteristics (e.g., age, cigarette and ECIG use, ECIG dependence) of all callers, those deemed ineligible, those deemed eligible, and study completers. Characteristics of those ineligible versus eligible were compared using inferential statistics (e.g., chi-square, independent samples $t$-tests). 
Predictors of ECIG dependence. Data from the telephone screening questionnaire were used to assess the ECIG use characteristics that may predict ECIG dependence. This questionnaire included items regarding cigarette use, ECIG use and ECIG dependence. A multiple linear regression was used for this aim to determine if ECIG dependence can be predicted based on these characteristics. Variables were examined to ensure they meet the assumptions of regression. First, variables were investigated for acceptable skew and kurtosis, using standards of $<3.2$ for skew/standard error skew (Zskew) and $<3.2$ for Zkurtosis (Kim, 2013). Variables were also assessed for a normal distribution through visual inspection of histogram. Next, $z$-scores for each variable were assessed to determine univariate outliers. Cases with $z$-scores $>3.2$ that seemed to be unrealistic were truncated or removed. When variables were not normally distributed, log and square root transformations were used, and the distribution was reassessed. Predictor variables also were investigated for their correlation with PSECDI scores and scatterplots between these variables were viewed to determine if there was a linear relationship. Finally, multicollinearity was assessed with the Variance Inflation Factor (VIF). Common rules of thumb have been that VIF $>10$ or $>4$ are problematic, however, there is no ideal standard that applies to all analyses (O’Brien, 2007).

All predictor variables initially considered for the model were continuous variables, including duration of use (months), frequency of use (days/week), liquid consumption (ml/day), nicotine concentration (mg/ml), device power (watts), and lifetime cigarette use. Age was included as a covariate in the model. All variables were entered into the model simultaneously. Significance of the full model and of individual predictors was determined at $p<0.05$. Specifically, the $F$-statistic $(p<.05)$ was reported to determine significance of the full model. $R^{2}$ was reported to explain how much variance in ECIG dependence can be accounted for by the 
model. Standardized beta coefficients were reported for individual predictors, which were considered significant predictors at $p<0.05$. An iterative approach to model assessment was used, in which the predictor with the largest $p$-value was removed and the model was run again. This approach was used until all individual predictors in the model were significant. Model fit was compared using the Akaike information criterion (AIC), with the lowest AIC value indicating the best model fit.

Based on previous research, it was hypothesized that all predictor variables would be positively associated with nicotine dependence. Frequency of use and duration of use were likely to be the strongest predictors given their reliable association with ECIG dependence. However, variables that influence nicotine delivery, such as nicotine concentration, device power, and liquid consumption may have complex interactions that limit their predictive validity as a single variable in the model. For example, individuals that use lower nicotine concentrations tend to vape at higher wattages and consume more liquid than individuals using higher nicotine concentrations (Smets et al., 2019).

Primary outcomes. Subjective and heart rate data were analyzed using a 2 (condition: ad lib, abstinent) x 7 (time, 8 for heart rate) repeated measures ANOVA. Huynh-Feldt corrections were used to adjust for violations of sphericity (Huynh \& Feldt, 1976). Differences between means were examined using Tukey’s Honestly Significant Difference (HSD; Keppel, 1991) and were reported significant at $p<.05$ level. It was hypothesized that withdrawal symptoms and heart rate will be lower, and nicotine effects will be higher, in the abstinent condition than the $a d$ lib condition. Additionally, these effects are expected to change over time in the abstinent condition while remaining stable in the ad lib condition. 
Secondary outcomes. Secondary outcomes were explored primarily with descriptive analyses rather than ANOVAs due to the limited power to detect significant differences associated with a small sample size. Secondary outcomes include cognitive tests (RVIP, $n$-back, Cued Go/No-Go) and the MCP. Additional descriptive analyses included percent change from time 0 for cognitive outcomes.

Two paired-samples $t$-tests were used for cognitive outcomes and the MCP to make planned comparisons $(p<0.05)$. Specifically, one $t$-test was used to compare the abstinent and ad lib conditions at $120 \mathrm{~min}$, and one $t$-test was used to compare the abstinent group at $0 \mathrm{~min}$ (baseline) and $120 \mathrm{~min}$. Both of these comparisons provided information regarding possible abstinence-induced cognitive changes and were used to generate effect sizes to inform future research. To correct for multiple comparisons, the False Discovery Rate procedure was used (Benjamini \& Hochberg, 1995). This procedure reduces the likelihood of making a Type I error by controlling for the expected proportion of false positives out of all rejected null hypothesis. This procedure may increase power to detect significant differences as compared to more conservative approaches such as the Bonferroni correction. The False Discovery Rate (FDR) procedure involved ranking all statistical tests followed by the determination of a new critical value for each test. It was hypothesized that cognitive performance would be poorer and MCP crossover point would be higher at 120 min of abstinence compared to baseline abstinence or 120 min ad lib ECIG use. Additionally, means and standard deviations were reported for session 1 and session 2 to determine if order effects may have influenced results.

Puff topography. Puff topography was characterized using puff number, puff duration, and IPI. Two independent raters scored these variables for each ad lib session. Interrater reliability was assessed for puff duration and IPI using two-way random Intraclass Correlation 
(ICC) for consistency among average measures, with values $\geq 0.90$ being considered reliable. Interrater reliability was determined for puff number using Fleiss’ Kappa for Interrater agreement.

Puff topography was characterized according to puff clusters according to a definition from recent work (St. Helen et al., 2016). This analysis was a way of assessing how individual puffs group together, rather than solely assessing each puff alone. Specifically, a puff cluster was defined as the number of puffs with no more than $60 \mathrm{sec}$ between each puff $(\leq 60 \mathrm{sec}$ IPI $)$. These clusters were further categorized by the number of puffs in a cluster: short ( $2-5$ puffs $)$, medium $(6-10$ puffs $)$ and long (>10 puffs). We modeled this approach using topography data from the ad lib session. Additional variables of interest were total puff number, average puff durations, average IPIs, the percent of total puffs within a session by cluster type, the number of puffs within each cluster type, and the IPI and number of puffs between clusters and the following up. Individual patterns of puffs also were examined for three participants that demonstrated different patterns. Video errors led to the loss of topography data for two participants.

Predicting withdrawal from puff topography. Puff topography variables were used to determine if topography is predictive of withdrawal outcomes. Specifically, the number of puffs, puff clusters, and average puff duration and IPI were measured for each 30-min timepoint (e.g., 0 - $30 \mathrm{~min}, 30$ - $60 \mathrm{~min}$, etc.). Multiple linear regressions were used to determine if these topography variables could predict primary outcomes (subjective withdrawal, nicotine effects, heart rate). Significance of the full models and of individual predictors was determined at $p<$ 0.05. Specifically, the $F$-statistic $(p<.05)$ was reported to determine significance of the full model. $R^{2}$ was reported to explain how much variance in ECIG dependence can be accounted for by the model. Standardized beta coefficients were reported for individual predictors, which was 
considered significant predictors at $p<0.05$. It was hypothesized that larger puff numbers and longer puff durations will be associated with lower subjective withdrawal, higher nicotine effects, and higher heart rate.

\section{Chapter 3 - Results}

\section{Feasibility and Recruitment}

Figure 5 demonstrates progress across the study phases of screening, enrollment, and completion. Of 178 individuals who called the laboratory and completed a telephone screening questionnaire, $41(23.03 \%)$ were deemed eligible. While $22(53.66 \%)$ of these 41 individuals were enrolled (i.e., consented), 15 (36.58\%) passed the in-person screening and actually began the study. Eleven of these 15 participants $(73.33 \%)$ completed the entire protocol. Also shown in this figure are reasons for ineligibility at telephone screen, with the most common reasons being PSECDI $<7(41.61 \% ; M$ score $=3.61, S D=2.15)$, past month marijuana use $>10(32.12 \%)$, and past year cigarettes $>10(20.44 \%)$. [Note that these reported percentages do not exclude participants also ineligible for other reasons.] Twenty-five callers $(18.25 \%$ of all callers, $43.86 \%$ of callers excluded for PSECDI < 7) were excluded for low PSECDI scores alone. These reasons for ineligibility were also the most common based on the in-person screening, with responses for PSECDI scores and marijuana use changing so that participants were no longer eligible. There were a large number of scheduled screenings and sessions that were not attended $(37.14 \%$ and $40.48 \%$, respectively).

Table 3 demonstrates demographic, cigarette use, and ECIG use characteristics for all callers $(n=178)$, ineligible callers $(n=137)$, eligible callers $(n=41)$, and completers $(n=11)$. A lower percentage of eligible callers were lifetime cigarette smokers compared to ineligible callers $\left(X^{2}(1)=4.15, p<.05\right)$. Those who were eligible for the study had higher PSECDI scores $[t(169)$ 
$=3.08, p<.01]$ and used ECIGs more days/week $[t(169)=2.08, p<.05]$ than ineligible callers, though these groups did not differ significantly on any other characteristic $\left(t^{\prime} s<1.66, X^{2}{ }_{s}<\right.$ 3.24, $p$ 's $>.05)$.

Screened callers. Overall, callers were mostly young adults $(M=20.96$ years, $S D=$ 4.14), and primarily nonsmokers (< 100 cigarettes lifetime, $72.60 \%)$. Most callers were daily ECIG users and preferred mint/menthol (46.5\%) and fruit (25.3\%) flavors. There was a high percentage of users of $4^{\text {th }}$ generation pod ECIGs $(76.69 \%$; with $60.74 \%$ reporting use of Juul specifically), which may account for the relatively high nicotine concentrations reported by the sample $(M=42.14 \mathrm{mg} / \mathrm{ml}$; mode and median $=59 \mathrm{mg} / \mathrm{ml})$. Indeed, for $4^{\text {th }}$ generation relative to $2^{\text {nd }} / 3^{\text {rd }}$ generation device users, higher nicotine concentrations $[M(S E)=54.43(1.06)$ vs. 7.37 (1.33) $\mathrm{mg} / \mathrm{ml} ; t(161)=-22.81, p<.001]$, less liquid $\mathrm{ml} /$ day [0.65 (0.07) vs. $6.53(1.10) \mathrm{ml} ; t(159)$ $=5.31, p<.001)]$, lower propylene glycol percentages $[30.69 \%(0.37)$ vs. $43.50 \%(3.66) ; t(136)$ $=3.48, p<.001]$, and lower wattages [11.16 (1.42) vs 70.61 (3.99) watts; $t(155)=13.97, p<$ $.001]$ were reported. Flavor preferences also differed by device type, with mint $(60.80 \%)$ and fruit $(20.80 \%)$ being the most common flavors in $4^{\text {th }}$ generation users and fruit $(53.57 \%)$ and sweet/dessert $(46.43) \%$ being preferred among $2^{\text {nd }} / 3^{\text {rd }}$ generation users, $X^{2}(5)=62.59, p<.001$. These $4^{\text {th }}$ generation users were also younger [19.84 (0.24) vs. $24.18(1.09)$ years, $t(161)=4.15$, $p<.001]$ and had a smaller percentage of lifetime smokers $\left(22.76 \%\right.$ vs. $42.11 \% ; X^{2}(1)=5.47, p$ $<.05$. However, these $4^{\text {th }}$ generation users also did not use ECIGs for a significantly shorter duration than $3^{\text {rd }}$ generation users [16.39 (2.47) vs. $23.84(2.95)$ months; $\left.t(160)=1.55, p>.05\right]$, or have significantly lower dependence scores [8.20 (0.41) vs. $9.18(0.70) ; t(169)=1.18, p>$ $.05]$. 
Study completers. Table 4 shows data for these and additional characteristics for completers, based on the information collected at the in-person screening visit only. Characteristics of completers were similar to that of all callers, though there was a higher percentage of nonsmokers $(90.90 \%)$ and higher PSECDI scores $(M=11.91, S D=3.30)$ for completers. All participants met the clinical definition of tobacco use disorder based on the MINI, with most participants classified as severely dependent (63.6\%), and others being split evenly between moderately (18.2\%) and mildly (18.2\%) dependent. These categorizations differ somewhat from those for the PSECDI, in which 36.4\% had high dependence, $45.5 \%$ had medium dependence, and $18.2 \%$ had low dependence. Additionally, $36.37 \%$ of participants had a higher MINI severity than PSECDI severity, with three of four of these participants deemed severe by MINI but moderate by PSECDI. In contrast, $18.18 \%$ had higher PSECDI severity than MINI severity, and $45.45 \%$ were classified the same by the two measures. There was not a significant correlation between PSECDI scores and the number of MINI symptoms, $r(9)=0.55, p=.08$. Similar to the sample of all callers, completers who used $4^{\text {th }}$ and $3^{\text {rd }}$ generation devices used different nicotine concentrations [56.63 (6.72) vs. $4.00(1.73) \mathrm{mg} / \mathrm{ml}]$, wattages [8.00 (0.00) vs. $70.00(17.32)$ watts], liquid use [0.55 (0.26) vs 8.00 (3.46) $\mathrm{ml} /$ day] and duration of use [1.05 (0.47) vs. 3.67 (2.08) years]. The higher proportion of $4^{\text {th }}$ generation users explains the higher nicotine concentration $(42.27 \mathrm{mg} / \mathrm{ml})$ among completers, though liquid use/day and wattage values are elevated due to the much higher levels among $3^{\text {rd }}$ generation users.

\section{Predictors of Dependence}

A multiple linear regression was used to determine if cigarette and ECIG use can predict ECIG dependence in the sample of 178 individuals screened via telephone. Initial predictors considered for the model were lifetime cigarette use, ECIG nicotine concentration $(\mathrm{mg} / \mathrm{ml})$, 
frequency of ECIG use (days/week), quantity of ECIG use (ml/day), duration of ECIG use (months), and device power (watts). Table 5 includes information regarding descriptive statistics for predictor variables and their correlation with PSECDI scores. Additional details regarding exploration and handling of variables, associations with dependence, and considered interactions between variables are presented in Appendix J.

As shown in Table 6, multiple linear regression models were run to predict PESCDI scores based on smoking status (dichotomous), nicotine concentration (continuous), daily use (dichotomous), log-transformed liquid/day (continuous), square root-transformed duration of use (continuous), log-transformed wattage (continuous) and log-transformed age (continuous) as a covariate. AIC was used to compare how well the models fit the data, with lower AICs being favorable. At each step, while the overall model was significant, not all individual predictors significantly predicted PSEDCI scores and models were rerun removing the predictor with the highest $p$-value. In model 1, VIF was 5.09 for nicotine concentration and 3.94 for wattage, which indicated the possibility for problematic multicollinearity. Due to this concern of multicollinearity, the model was rerun removing nicotine concentration alone (Model 2) and wattage alone (Model 3). Model 2 had a lower AIC than model 3 which indicated a better fit to the data, so subsequent models were run based on Model 2. In subsequent models, liquid use was removed (Model 4), nicotine concentration was removed (Model 5), and smoking status was removed (Model 6). In this final model, both remaining variables significantly predicted PSECDI scores, with daily ECIG use and longer duration of ECIG use being associated with higher dependence. However, as variables were being removed, models had higher AICs, demonstrating a worse fit. One additional model was attempted (Model 7), removing smoking status (variable with highest $p$-value) from model 1. The AIC for Model 7 was higher than for Model 1, so 
Model 1 was determined to best fit the data. In Model 1, increased age, nicotine concentration, daily ECIG use, duration of ECIG use, and wattage were significantly associated with higher ECIG dependence. However, beta weights and standard errors for nicotine concentration and wattage should be interpreted with caution this model should be interpreted with caution due to potential multicollinearity issues.

\section{Heart Rate}

Table 7 shows main effects and interaction results from repeated-measures ANOVAs for heart rate and subjective outcomes. There was a significant main effect of condition and of time for heart rate. Specifically, heart rate was significantly lower in the abstinent condition $(M=$ $73.59, S E=1.76)$ than in the $a d$ lib condition $(M=78.59, S E=2.15)$. For the main effect of time, heart rate at baseline (post-directed bout, $M=86.11, S E=2.17$ ) was significantly greater than all other timepoints: pre-directed $(M=77.32, S E=1.75), 30 \min (M=76.03, S E=1.88), 60$ $\min (M=74.25, S E=1.94), 90 \min (M=75.00, S E=2.19), 120 \min (M=74.23, S E=2.26)$, $150 \min (M=72.60, S E=1.57)$ and $180 \min (M=72.95, S E=1.94)($ Tukey's HSD; $p<.01)$.

Figure 6 demonstrates heart rate over time for both sessions.

\section{Subjective Questionnaires}

MNWS. A significant condition $\mathrm{x}$ time interaction was observed for craving $(F=4.04, p$ $<.05$; Figure 7$)$. Post-hoc tests revealed that ratings of craving were lower at baseline $(M=$ $20.09, S E=5.51)$ than at $120 \min (M=55.73, S E=8.49, p<.05), 150 \min (M=64.09, S E=$ $8.39)$, and $180 \min (M=68.91, S E=8.08, p$ 's $<.01)$ within the abstinent condition, but not lower than $30 \min (M=28.55, S E=7.20), 60 \min (M=38.64, S E=7.45)$ or $90 \min (M=43.09$, $S E=7.32$ ). No significant differences were observed across timepoints within the ad lib condition, or between conditions. A similar pattern was observed for urges, which was also 
significant for condition $\mathrm{x}$ time $(F=2.53, p<.05)$. Post-hoc tests revealed that ratings of urges at baseline $(M=24.36, S E=7.13)$ were significantly lower than at $120 \min (M=54.36, S E=8.82)$, $150 \min (M=65.18, S E=9.01)$ and $180 \min (M=69.36, S E=8.07, p$ 's $<.01)$ in the abstinent condition, but not lower than $30 \min (M=40.09, S E=7.26), 60 \min (M=38.55, S E=6.78)$ or $90 \min (M=48.91, S E=7.93)$. Additionally, urges were significantly different at $180 \mathrm{~min}$ between the abstinent and $a d$ lib conditions $(M=37.36, S E=7.62, p<.01)$. Also observed were significant main effects of time for the items difficulty concentrating, impatient, and irritable $(F$ 's $>3.21 ; p$ 's $<.05)$; however, post-hoc tests revealed that these effects were not reliable. For impatient, for instance, the item with the largest $F$ value for the main effect of time, baseline values $(M=8.32, S E=2.68)$ were not significantly lower than at $30 \min (M=17.45, S E=4.25)$, $60 \min (M=13.82, S E=3.45), 90 \min (M=15.05, S E=4.16), 120 \min (M=19.91, S E=5.28)$, $150 \min (M=29.46, S E=7.99)$ or $180 \min (M=30.05, S E=7.89)$. No other main or interaction effects were observed for the remaining items (all $p$ 's $>.05$ ).

Tiffany-Drobes QSU-Brief. A significant condition x time interaction was observed for Factor 1 (intention to smoke; Figure 8). Post-hoc tests revealed that Factor 1 scores were lower at baseline $(M=11.09, S E=2.02)$ than at $90 \min (M=20.18, S E=2.16, p<.05), 120 \min (M=$ 21.64, $S E=2.18), 150 \min (M=21.91, S E=2.35)$ and $180 \min (M=23.18, S E=2.17, p$ 's $<$ $.01)$ in the abstinent condition, but not lower than $30 \min (M=14.82, S E=2.32)$ or $60 \min (M=$ $17.36, S E=2.36)$. No significant differences were observed between timepoints within the $a d l i b$ condition, or between conditions. For Factor 2 (anticipation of relief from withdrawal), significant main effects of condition and time were observed. Factor 2 scores were higher in the abstinent condition $(M=3.75, S E=0.99)$ than in the ad lib condition $(M=2.65, S E=0.70)$. Post-hoc tests were not reliable for the main effect of time for Factor 2. 
DENS. There was a significant condition $\mathrm{x}$ time interaction for weak $(F=3.22, p<.05$; Figure 9), with ratings higher at baseline $(M=17.55, S E=7.51)$ than at $60 \min (M=1.82, S E=$ 0.92), $90 \min (M=0.91, S E=0.32), 120 \min (M=1.00, S E=0.38), 150 \min (M=1.09, S E=$ $0.50)$, and $180 \min (M=0.91, S E=0.55)$ in the abstinent condition (Tukey's HSD, $p$ 's $<.05)$, but not $30 \min (M=9.36, S E=5.26)$. There were no significant differences between $a d$ lib and abstinent conditions at any timepoint. There were significant main effects of time for dizzy, heart pounding, and lightheaded $\left(F{ }^{\prime} s>4.45, p\right.$ 's $\left.<05\right)$; however, post-hoc tests did not demonstrate significant differences between any timepoints for these outcomes. For lightheaded for instance, the item with the larges $F$ value for the main effect of time $(F=5.98)$, baseline values $(M=$ 34.46, $S E=10.60)$ were not significantly higher than at $30 \mathrm{~min}(M=18.50, S E=7.65), 60 \mathrm{~min}$ $(M=13.50, S E=4.74), 90 \min (M=8.41, S E=3.19), 120 \min (M=7.41, S E=3.20), 150 \min$ $(M=5.91, S E=2.46)$, or $180 \mathrm{~min}(M=6.77, S E=2.77$. There were no significant main effects or interactions for any of the other DENS items.

DEPS. Significant condition x time interactions were observed for the DEPS items of pleasant and satisfying $(F$ 's $>3.61, p$ 's $<.05)$. For pleasant, average ratings were $72.00(S E=$ 5.98) at baseline, $43.18(S E=11.72)$ at $30 \mathrm{~min}, 47.91(S E=13.51)$ at $60 \mathrm{~min}, 50.09(S E=14.11)$ at $90 \mathrm{~min}, 50.82(S E=14.46)$ at $120 \mathrm{~min}, 52.46(S E=14.65)$ at $150 \mathrm{~min}$, and $54.18(S E=14.80)$ at $180 \mathrm{~min}$ in the abstinent condition (Figure 10). However, post-hoc tests revealed a significant difference between baseline and 30 min only (Tukey's HSD, $p<.05$ ). No differences were observed between timepoints within the ad lib condition, and ratings were significantly higher at the 30-min timepoint for ad lib $(M=74.18, S E=7.32)$ relative to abstinent conditions (Tukey's $\mathrm{HSD}, p<.01)$. For satisfying, average ratings were $70.91(S E=5.96)$ at baseline, $44.00(S E=$ $10.80)$ at $30 \mathrm{~min}, 46.64(S E=13.21)$ at $60 \mathrm{~min}, 49.27(S E=13.99)$ at $90 \mathrm{~min}, 50.27(S E=14.37)$ 
at $120 \mathrm{~min}, 52.36(S E=14.31)$ at $150 \mathrm{~min}$, and $52.35(S E=14.37)$ at $180 \mathrm{~min}$ in the abstinent condition. However, post-hoc tests revealed significant differences between baseline to $30 \mathrm{~min}$ and 60 min only (Tukey's HSD, $p<.05$ ). No significant differences were observed between timepoints within the ad lib condition, and ratings were significantly higher at the 30-min timepoint for $a d$ lib $(M=77.64, S E=5.63)$ relative to abstinent conditions (Tukey's HSD, $p<$ .01). There was a significant main effect of time for calm, however, post-hoc tests were not reliable. There were no significant main effects or interactions for any of the other DEPS items.

\section{Randomization and Training}

Randomization to condition resulted in five participants receiving the ad lib session first and six participants receiving the abstinence condition first. Randomization was matched based on RVIP percent correct at screening, which was demonstrated to be successful: $54.60 \%$ ( $S E=$ $6.48)$ versus $54.83 \%(S E=7.98)$ for these groups, respectively, $t(9)=-0.02, p=0.98$. During training, the number of practice tasks completed was $3-4(M=3.18, S D=0.41)$ for the RVIP, 2 $-3(M=2.45, S D=0.52)$ for the $n$-back, and $1-2(M=1.09, S D=0.30)$ for Cued Go/No-Go. Table 8 describes the best performance on cognitive tasks during training for all completers.

\section{Cognitive Tasks}

Table 9 demonstrates $t$-test results for all cognitive outcomes. Table 10 shows mean cognitive task performance for both conditions at baseline and $120 \mathrm{~min}$, and Table 11 shows means and standard deviations for change in cognitive performance from zero min to $120 \mathrm{~min}$ in each condition.

RVIP. As shown in Table 9, no RVIP-related outcomes showed significant differences between timepoints (all $p$ 's $>.05$ ). Figure 11 demonstrates mean and standard error for percent correct for the $a d \mathrm{lib}$ and abstinent sessions at baseline and $120 \mathrm{~min}$. 
$N$-back. A paired-samples $t$-test demonstrated significantly better 3-back accuracy at 120 min abstinence $(M=69.00, S E=6.49)$ than baseline abstinence $(M=51.09, S E=5.95 ; \mathrm{FDR}, p$ $<.005$; Figure 12). There were no other significant differences for any $n$-back outcomes (Table 9).

Cued go/no-go. Incongruent accuracy was poorer in the abstinence condition at 120 min $(M=97.36, S E=0.51)$ compared to baseline $(M=99.00, S E=0.40), t(10)=-3.99, p=.003, d=$ 1.08 (FDR, $p<.005$; Figure 13). Incongruent accuracy was also poorer at $120 \mathrm{~min}$ abstinence compared to $120 \mathrm{~min}$ ad lib use $(M=98.73, S E=0.41), t(10)=-2.68, p=.023, d=0.90$, however, this finding did not remain significant after the FDR procedure for multiple comparisons. There were no other significant differences for any cued go/no-go outcomes (Table 9).

Order effects. Cognitive tasks were investigated to determine if session order influenced cognitive performance. Though no inferential tests were run, Table 12 demonstrates means and standard deviations for all cognitive outcomes for both sessions and timepoints. Visual inspection of the means does not suggest any clear order effects across outcomes.

\section{Multiple Choice Procedure}

Table 13 demonstrates crossover points for each session and timepoint. A paired samples $t$-test demonstrated a significantly higher crossover point at $150 \mathrm{~min}$ abstinence $(M=2.15, S E=$ $0.50)$ compared to baseline abstinence $(M=1.51, S E=0.40), t(10)=2.38, p=.039, d=0.42$, however, this finding was not significant after the FDR procedure for multiple comparisons. There was not a significant difference in crossover point between $150 \mathrm{~min}$ abstinent and $150 \mathrm{~min}$ ad lib use, $t(10)=1.93, p=.083, d=0.23$. 


\section{Puff Topography}

Interrater reliability coefficients were high for puff duration $(\mathrm{ICC}=0.93, p<.001)$ and for IPI $(\mathrm{ICC}=1.00, p<.001)$. Interrater agreement was high for puff number $(\mathrm{kappa}=1.00, p=$ $.001)$.

Participants took an average of 51.44 puffs $(S D=20.93$, Range $=17-77$, Median $=58)$ in the 180-min ad lib session. Average puff durations for participants were $2.76 \mathrm{sec}(S D=1.24$, Range $=1.13-4.58$, Median $=2.53)$ with an average IPI of $239.23 \mathrm{sec}(S D=107.56$, Range $=$ 137.41 -468.83 , Median =184.54). Table 14 demonstrates the clustering patterns of each participant. This session consisted of $41.93 \%$ single puffs $(S D=27.73$, Range $=0.00-88.24 \%$, Median $=45.76 \%)$, for an average of 17.33 puffs for this puff type $(S D=9.71$, Range $=0-28$, Median $=19)$. For clustered puffs, $49.37 \%$ were deemed short $(S D=21.86$, Range $=11.76-$ $76.92 \%$, Median $=54.24 \%), 7.75 \%$ were deemed medium $(S D=12.00$, Range $=0.00-29.87 \%$, Median $=0.00 \%$, and $0.0 \%$ were deemed large. The average number of puff clusters per session was $11.00(S D=6.67$, Range $=1-21$, Median $=12)$, with an average of 10.33 small clusters $(S D=6.46$, Range $=1-21$, Median $=12)$ and 0.67 medium clusters $(S D=1.12$, Range $=0-3$, Median $=0$ ). All participants had at least 1 short puff cluster, though only three participants had a medium cluster. The average time between a cluster and the following puff was $356.46 \mathrm{sec}(S D$ $=156.60$, Range $=185.56-661.53$, Median $=339.69)$, with an average of 1.70 puffs $(S D=1.16$, Range $=0.2-3.2$, Median $=1.56$ ) between each cluster. In comparison, the average time between a single puff and the following puff was $338.91 \mathrm{sec}(\mathrm{SD}=202.50$, Range $=189.88-$ 819.00). There was an average of 2.71 puffs $(S D=0.61$, Range $=2-3.85$, Median $=2.44)$ within short clusters and an average of 6.56 puffs $(S D=0.96$, Range $=6-7.67$, Median -6.00$)$ within medium clusters. 
Figures 14-16 demonstrate puffing patterns for three individual participants: 501, 507, and 513. These participants were chosen to represent of the wide variation in puffing patterns across participants. Participant 501 was an individual who took relatively many puffs in the $a d$ lib condition (65), 95.38\% of which were taken in clusters. Participant 507 was an individual who took relatively few puffs (17), and $88.24 \%$ of these puffs were single puffs. Participant 513 was an individual who took a relatively moderate number of puffs in the ad lib condition (50), which were somewhat split between single (56.00\%) and clustered puffs $(44.00 \%)$. In addition to these puffing patterns, Figures 17-19 demonstrate puffs plotted with heart rate and ratings of urges from the MNWS. It is difficult to discern a clear pattern when viewing these graphs. For example, participant 501 takes few puffs and has a large increase in ratings of "urges" from 0 30 minutes, but these ratings stay fairly stable after decreasing between $30-60$ minutes regardless of puffing. Participant 507 reports having no urges to vape (i.e., rating of zero) at 30 minutes despite taking no puffs since the standardized bout at the beginning of session, and their ratings seemingly increase when more puffs are taken (60 - 90 minutes) and decrease when fewer puffs are taken $(120-150$ minutes $)$. The trends for participant 513 are even less clear, especially considering their ratings of "urges" stay stable across the session despite taking many puffs from 150 - 180 minutes. Generally, heart rate fluctuates for all participants but stays stable around a consistent mean. Taken together, these graphs suggest that there is a large amount of variability between- and within-participants in these outcomes, and differences may be difficult to detect without the use of statistical techniques.

\section{Predicting Withdrawal from Topography}

Multiple linear regressions were conducted to determine if withdrawal and nicotinerelated outcomes could be predicted based on puff topography in the ad lib session. Total puff 
number, average puff duration, average IPI, and the number of puff clusters were entered into the model as predictor variables. These variables were grouped into 30-minute bins (e.g., 0 - 30 minutes, $30-60$ minutes, 60 - 90 minutes, etc.) and paired with the outcome variable at the end of that time period (e.g. heart rate at 30 minutes, 60 minutes, 90 minutes, etc.). Multicollinearity was assessed due to the high correlation between puff number and the number puff clusters. VIF was 4.33 for puff number and 3.70 for puff clusters. It is difficult to determine if these values indicate problematic multicollinearity (one rule of thumb is VIF $>4$ ), so significant models were rerun twice removing puff number or puff clusters from the models individually. Situations in which the model changed upon removing puff number of puff clusters are reported in text. However, due to the exploratory nature of these analyses, the full regression models for all outcomes are presented in Table 15.

Heart rate. Heart rate was predicted significantly based on topography variables (Table 15). Specifically, longer puff durations and more puff clusters were associated with lower heart rate. Removal of puff clusters from the model led to the model no longer significantly predicting heart rate. However, the model remained significant when puff number was removed and longer puff durations again were associated with lower heart rate.

MNWS. Craving, drowsy, hunger, impatient, and irritable were significantly predicted by puff topography (Table 15). Notably, impatient had the largest variance accounted for $\left(R^{2}=\right.$ 0.42) of all models. Longer puff durations were associated with increased ratings of craving, drowsy, hunger, impatient, irritable, as well as anxious and restless though those two full models were not significant. Longer IPIs were associated with increased ratings of craving and urges, though the full urges model was not significant. Longer IPIs were also associated with reduced ratings of drowsy. Increased puff number was associated with reduced ratings of irritable, and 
more puff clusters were associated with increased ratings of hunger and irritable. Removal of puff number or puff clusters led to irritable no longer being significantly predicted by puff topography. For impatient and craving, removing puff clusters led to higher puff numbers being associated with higher ratings. All other models remained significant when puff number or puff clusters were removed from the model. Models for other MNWS were not significantly predicted by puff topography variables.

Tiffany-Drobes QSU-Brief. Neither Factor 1 nor Factor 2 were predicted significantly by puff topography (Table 15), though longer puff durations were associated with higher ratings of Factor 2.

DENS. Ratings of nervous and excessive salivation were significantly predicted by topography variables (Table 15). Increased puff number was associated with lower ratings of nervous and more puff clusters were associated with increased ratings of nervous and excessive salivation. The nervous model was no longer significant when puff number was removed, and the nervous and salivation models were no longer significant when puff clusters were removed. No other DENS models were predicted significantly by puff topography, though more puff clusters were associated with increased ratings of confused.

DEPS. Ratings of awake, concentrate, pleasant, reduced hunger, satisfying, and taste good were significantly predicted by puff topography. However, no individual puff topography variables predicted awake, pleasant, satisfying, or taste good. Shorter puff durations were associated with higher ratings of concentrate and reduced hunger, and shorter IPIs were associated with higher ratings of reduced hunger. For satisfying, pleasant, and taste good, removal of puff clusters led to a positive association between puff number and these items, and 
removal of puff number led to a positive association between puff clusters and the items. No other DEPS items were predicted significantly by puff topography.

\section{Chapter 4 - Discussion}

\section{Overview of Study Aims}

This study is the first to assess short-term withdrawal in experienced ECIG users that are primarily nonsmokers. That is, previous work assessing withdrawal has included ECIG users who are current or former cigarette smokers (Dawkins \& Corcoran, 2014; Hughes et al., 2019a), thus presenting a confound. In this study, withdrawal was assessed using a within-subjects design in which participants abstained from ECIG use or used their own ECIG ad lib for 180 min. Primary aims were to: a) assess feasibility of the current study; b) determine characteristics that predict ECIG dependence; and c) assess the effects of $180 \mathrm{~min}$ of abstinence versus 180 min of ad lib ECIG use on heart rate and subjective withdrawal and nicotine effects. Secondary aims were to: a) assess the effects of abstinence and ad lib ECIG use on measures of cognition and ECIG reward; b) characterize puff topography during $180 \mathrm{~min}$ of ad lib ECIG use; and c) explore if puff topography variables are predictive of withdrawal and nicotine effects experienced during ad lib ECIG use.

\section{Feasibility and Recruitment}

The number of individuals determined to be eligible among those who completed the telephone screening questionnaire was low (23.03\%). A primary reason for ineligibility was relatively low scores on the PSECDI $(<7)$, with $18.3 \%$ of callers being excluded for this reason alone and $41.6 \%$ of callers being excluded for this and other reasons. While this criterion was chosen in an effort to exclude those individuals least likely to be ECIG dependent, the PSECDI scale has not been validated. Perhaps telling is that, for those who completed the study, a lower 
proportion were deemed "highly" dependent using the PSECDI than using the MINI for Tobacco Use Disorder (i.e., 36.4\% vs 63.6\%, respectively). Another common reason for ineligibility was marijuana use $(32.12 \%)$, but the use of this criterion is important given the potential effects of acute or chronic marijuana use on cognitive functioning (for review, see Crean, Crane, \& Mason, 2011). Many participants also were excluded due to their past year and/or past month cigarette use $(32.12 \%)$. This proportion of ECIG users with relatively little cigarette smoking history is comparable to that reported in recent samples of young adults $(69 \%-78.4 \%$ for college students, Ickes et al., 2019; Leavens et al., 2019;), the age range for the majority of those screened and completed here. This pattern is further supported by recent work with college-aged Juul users, of which only $4.1 \%$ reported former cigarette smoking (Leavens et al., 2019). Interestingly, eligible and ineligible participants did not differ on most baseline characteristics related to cigarette and ECIG use history. Besides a higher PSECDI score and lower rates of cigarette use, eligible participants differed from ineligible participants only in that they reported more days/week of ECIG use.

Another hindrance to enrollment rates involved the high proportion of eligible callers who failed to respond to requests to schedule an in-person screening visit (46.34\%). These individuals may have decided that they were not interested in participation, or may not have been responsive to our methods of communication. Early in the study, we relied on the use of telephone-based correspondence; however, use of digital technology such as texting, e-mail, and online screening questionnaires have been argued as more acceptable communication methods among younger generations (Dalessandro, 2018; Haste, 2005). While we did not target this younger age group for participation, they appear to be those most likely to fit our criteria. 
Consequently, email and text messaging forms of communication were later offered as an option to individuals interested in participation.

Of those who appeared eligible based on the telephone screen $(n=41)$, twenty-two (53.7\%) showed for the in-person screening and were therefore consented for the study. However, seven $(31.82 \%)$ of these individuals were ultimately deemed ineligible based on the in-person screening procedures and did not begin the study. The primary reason for ineligibility at this stage was individuals' discrepant responses between the telephone phone screening and in-person screening questionnaires. For the 15 participants that began the study, $11(73.33 \%)$ completed both sessions. For most individuals who failed to attend scheduled screening (37.1\%) or session (40.5\%) appointments, reasons for nonattendance were generally unknown. Unfortunately, the published literature rarely details eligibility or enrollment rates, as well as reasons for dropout or non-attendance (e.g., Dawkins, Turner, Hasna, \& Soar, 2012; Hendricks et al., 2006; Parrott et al., 1996; Wesnes et al., 2013), making it difficult to determine if these rates are unique to the current study.

Some of the encountered difficulties may be a result of the young adult, college student sample (63.6\% college students) that participated in the study (for review, see Khatamian Far, 2018). Characteristics of young adult populations include poor impulse regulation, delay of gratification, and vulnerability to peer influence (Steinberg, 2007), which may be barriers to regular participation in a research study. College students self-report scheduling conflicts and lack of time as logistical conflicts to participation (Davidson et al., 2010; Khatamian Far, 2018). These issues may be particularly relevant for the current study, in which experimental sessions required participants to have at least 3.5 hours of uninterrupted time. In the current study, $57.1 \%$ of college students took longer than 2 weeks to complete the study (median $=16$ days), as 
compared to only $25 \%$ of non-students (median $=8$ days). However, attempts to recruit noncollege community members via advertisements (e.g., fliers in vape shops) and online postings (Facebook vaping groups, Craigslist) were not highly successful and received lower response rates over time. Additionally, the recruitment strategies used for this study included those passive (fliers, emails) and active (attending classes, information booths), as recommended by others (Khatamian Far, 2018).

\section{Predictors of ECIG Dependence}

To evaluate the predictors of nicotine dependence, a convenience sample was used. Of the 178 individuals included in this sample, the large majority were college students and thus of relatively young age. Fourth generation pod systems were commonly used, such as Juul (similar to Ickes et al., 2019; Leavens et al., 2019). Not surprisingly, therefore, the average liquid nicotine concentration was high and the average power output was low. Also, the preferred liquid flavors were mint and fruit, which are the first and second most common flavors among college students who use Juul (Leavens et al., 2019), and the third and fourth most common flavors among adult ECIG users (Yingst, Veldheer, Hammett, Hrabovsky, \& Foulds, 2017). Approximately one-third of the sample reported smoking more than 100 cigarettes in their lifetime, though the majority of this subsample reported relatively low levels of cigarette use in the past month (e.g., $60.47 \%$ reported 0 cigarettes; $86.05 \%$ reported $<10$ cigarettes) and year (e.g., 31.42\% reported 0 cigarettes; $51.42 \%$ reported $<10$ cigarettes). The average PSECDI scores of this sample indicated moderate levels of ECIG dependence, equivalent to average PSECDI scores reported previously for a large sample of current ECIG users who were former cigarette smokers (8.53 versus 8.1, respectively; Foulds et al., 2015). Similar, or slightly higher scores, have been reported elsewhere (e.g., 8-12; Hiler et al., 2017; Hu et al., 2019; Leavens et al., 2019; Spindle 
et al., 2018) among experienced ECIG users, though these scores indicate low to moderate dependence and are notably low on a scale with a maximum score of 20 (Foulds et al., 2015).

The best model to predict ECIG dependence was that which included all predictors. In this model, older participants, higher nicotine concentrations, daily ECIG use, longer durations of use, and higher device wattages predicted higher ECIG dependence scores, though smoking status and volume of liquid/day did not. This pattern is consistent with previous work documenting positive associations between these same variables and ECIG dependence scores as measured by PSECDI (Foulds et al., 2015; Piper, Baker, Benowitz, Smith, \& Jorenby, 2019) or other measures (Johnson et al., 2018; Morean et al., 2018; Piper et al., 2019). Higher PSECDI scores have also been associated with the use of more advanced generation ECIGs (i.e., $2^{\text {nd }}$ and maybe $3^{\text {rd }}$ generation devices; Foulds et al., 2015; Yingst et al., 2015), and these devices typically have higher wattages. Higher wattages (Kosmider, Spindle et al., 2018) and higher nicotine concentrations (Hiler et al., 2017; Ramôa et al., 2016) are associated with increased nicotine delivery, which may promote greater dependence. No relationship was found between cigarette smoking and ECIG dependence, a finding supported by some work (Johnson et al., 2018) but not others (Morean et al., 2019). Daily volume of liquid used also was not a significant predictor (similar to Browne \& Todd, 2018), though ECIG consumption patterns are difficult to characterize (Blank et al., 2016; Pearson et al., 2018). Depending on the type of device used, individuals may rely on a myriad of behaviors to estimate their daily consumption: number of single puffs, number of clustered puffs, number of pods/cartridges/tanks, ml of liquid, etc. (Blank et al., 2016). For obvious reasons, some of these behaviors may be easier to estimate than others, and users may ultimately be inaccurate in their reporting. In support of this idea, the correlation between liquid consumption and the reported number of ECIG uses/day was notably low in the 
current study. For example, one caller reported using $32 \mathrm{ml}$ /day in 15-19 ECIG uses (15 puffs or 10 minutes of use), while another caller reported using $0.1 \mathrm{ml} /$ day in $30+$ daily ECIG uses.

These results should be interpreted with caution, as the full model only accounted for $18 \%$ of the variance in dependence scores. Also concerning is that the predictor variables revealed low correlations with the PSECDI scores, and were not normally distributed even after their transformation. Additionally, higher nicotine concentrations are used with lower wattages (Browne \& Todd, 2018; Smets et al., 2019) and were strongly negatively correlated in the current sample, which may have caused multicollinearity issues that impacted beta weights and inflated standard errors for those predictors. Daily ECIG use and use duration may have the most reliable relationships with ECIG dependence, as these predictors remained significant as nonsignificant predictors were removed. Though interaction variables were not used in these regressions due to violations of assumptions and unclear utility, it would benefit the field to continue to develop mathematical models regarding the interaction of device features and puff topography to predict nicotine yield (Hensel et al., 2019; Talih et al., 2015; Talih et al., 2017) and delivery. Continued assessment of ECIG characteristics, use behavior, and dependence will provide a comprehensive understanding of which factors of ECIG use are encouraging dependence among users.

\section{Abstinence Effects on Primary and Secondary Outcomes}

Another goal of this work was to compare subjective, physiological, cognitive, and behavioral outcomes between acute periods of ECIG abstinence and ad lib use. These analyses were conducted for those eleven participants who completed the entire study. Completers were primarily young adult nonsmokers $(90.9 \%)$ who used $4^{\text {th }}$ generation pods $(72.7 \%)$ with high average nicotine concentrations, and had a preference for mint (45.5\%) and fruit $(45.5 \%)$ flavors. 
They also had been using ECIGs daily for nearly two years on average, and had moderate-high levels of ECIG dependence.

Heart rate. For heart rate, significant increases were observed from pre- to post-directed bout in both conditions, suggesting that users were exposed to nicotine at the onset of sessions (similar to Hiler et al., 2017; Spindle et al., 2017; Spindle et al., 2018). Heart rate decreased thereafter for both conditions when examined across time, though to a greater degree for ECIG abstinence than for ad lib. Consequently, average heart rate was significantly lower for ECIG abstinence, when values were collapsed across time. A similar pattern of results has been observed in cigarette smokers undergoing a brief period of abstinence (Hendricks et al., 2006; Parrott et al., 1996). This effect may be a physiological indicator of nicotine withdrawal (Ward, Garvey, \& Bliss, 1992) or a nicotine offset effect (Shiffman, West, \& Gilbert, 2004; West \& Schneider, 1988). Offset effects are observed upon immediate removal of the drug, such as that experienced when an ECIG user stops puffing on their device. An example might be when heart rate declines because it is no longer increasing from the nicotine absorbed during ECIG puffing. These declines in heart rate may remain stable over a long period (e.g., 5 weeks in West \& Schneider, 1988), which may reflect a lack of nicotine rather than a temporary withdrawal effect.

Subjective effects. As for subjective effects, significant interactions between condition and time occurred for several items known to be reliable indicators of nicotine/tobacco withdrawal, specifically craving, urges to vape, and Factor 1 (intention to vape). For each of these items, significantly increased ratings were observed within 90 - 120 min after baseline for the abstinence but not the ad lib condition. Still, post-hoc tests failed to detect differences between timepoints when compared across conditions. A similar pattern was observed for some product-related effects (i.e., satisfying and pleasant), though ratings found to be significantly 
different from baseline were those earlier in the session (e.g., 30-60 min). Also observed were significantly higher ratings for Factor 2 (anticipation of relief from withdrawal) for the abstinence relative to ad lib condition (collapsed across timepoints), as well as lower ratings of various withdrawal- and product-related items (e.g., difficult concentrating, impatient, irritable, lightheaded, dizzy) at baseline relative to other timepoints (collapsed across conditions). For these latter effects, however, differences between timepoints were not significant based on posthoc tests. Cigarette smokers have shown similar changes in withdrawal symptoms as early as 30 min (e.g., craving, anxiety, difficulty concentrating; Hendricks et al., 2006), and as late as four hours (e.g., urges to smoke, irritability, difficulty concentrating; Parrott et al., 1996), during an acute period of abstinence. Importantly, the magnitude of ratings reported here is similar to that reported by Hendricks and colleagues (2006), as well as in other work that assessed withdrawal in experienced ECIG users following a period of overnight abstinence (Hiler et al., 2017; Spindle et al., 2017; Spindle et al., 2018).

A recently published study (Hughes et al 2019b) assessed longer-term ECIG withdrawal in a sample of experienced users with minimal cigarette smoking history $(<100$ cigarette uses in lifetime). Participants used their own ECIG device as usual for one week or no ECIG device for one week, and thus experienced a period of ad lib use and a period of abstinence. Withdrawal effects and heart rate were measured during these two weeks. Relative to the period of regular ECIG use, the abstinence period resulted in reduced heart rate and elevated withdrawal ratings. These effects peaked within the first two days of abstinence, and were most pronounced for the subjective items of craving and irritability. When making comparisons across studies, however, the withdrawal symptoms observed among this sample of non-smoking ECIG users appears reduced relative to ECIG users that are former smokers (Hughes et al., 2019a). This observation 
has led to the suggestion that ECIG withdrawal in non-smokers may not be of clinical or regulatory significance (Hughes et al., 2019a).

In a national dataset, $25 \%$ of exclusive ECIG users met the DSM-5 definition of withdrawal (experience 4+ withdrawal symptoms) when trying to quit, and only $40 \%$ of users experienced at least one withdrawal symptom (Hughes \& Callas, 2019). Less withdrawal symptoms were experienced by ECIG users trying to quit than cigarette smokers, however, not all ECIG users were daily users. Anger, anxiety, and eating more were the most common withdrawal effects reported, though many of the effects reported in the current study (craving, urges, impatient), were not assessed. Notably, this study involved a small sample of ECIG users $(n=25)$ and their history of cigarette use is unknown. It remains unclear if other measures of withdrawal (e.g., difficulty concentrating, restlessness) are not experienced by ECIG users as severely as cigarette smokers or if longer abstinence periods are needed for ECIG users to demonstrate these withdrawal effects. Future studies with a longer abstinence duration and a larger sample will provide additional insight into the primary symptoms and timecourse of ECIG withdrawal.

Cognitive effects. Given the low sample size, and thus the lack of statistical power, few significant effects were observed for the cognitive measures. Indeed, neither significant condition nor time effects were detected for any RVIP outcome. For cigarette smokers, deficits in RVIP reaction time have been observed by 30 min of abstinence (Hendricks et al., 2006) and RVIP accuracy after overnight abstinence (McClernon et al., 2008; Parrott \& Craig, 1992; Wesnes \& Warburton, 1984). Attention as measured by other tasks have also been sensitive to cigarette abstinence-induced changes in reaction time or accuracy within two to six hours 
(Parrott et al., 1996), 12 hours (Leventhal et al., 2010; McClernon et al., 2008; Myers et al., 2008; Parrott \& Craig, 1992), and 17 hours (Atzori et al., 2008).

For inhibitory control, No-Go Incongruent accuracy was decreased significantly at the end of the abstinence condition (120 min), relative to baseline for abstinence and also relative to the end of the ad lib condition (120 min). No-Go Incongruent trials may be a particularly robust measure of inhibitory control, as errors of commission for No-Go trials have shown to increase in cigarette smokers after 24 hours of abstinence (Hatsukami et al., 1989; Kozink, Kollins et al., 2010). Also shown in cigarette smokers are deficits in reaction times on this same task within 5 hours (Harrison et al., 2019) and 8-12 hours (McClernon et al., 2008) of abstinence. In this study, reaction time was not influenced by condition or time.

The only significant finding for the n-back task was a significant difference between timepoints within the abstinence condition for 3-back accuracy. Specifically, accuracy was significantly higher at 120 min compared to baseline for this condition. A similar pattern was observed for the ad lib condition, though the finding was not significant. It is possible that participants improved with repeated administrations of the 3-back task, or that the 3-back version administered at 120 min was easier than the version administered at baseline. In comparison, cigarette smokers reliably demonstrate deficits in working memory accuracy and reaction time at 6 hours (Grundey et al., 2015), 12- 13 hours (Mendrek et al., 2006; Myers et al., 2008), and 24 hours of abstinence (Falcone et al., 2014; Jacobsen et al., 2005).

Study results for cognitive outcomes are not likely due to condition order, as randomization based on RVIP performance at screening was successful. Still, more extensive training might be warranted. Studies involving cognitive assessments have used different training procedures, such as training to stability or a specific criterion (Ernst et al., 2001; Kozink, Lutz et 
al., 2010; Mendrek et al., 2006), multiple training sessions (Gilbert et al., 2005; Kozink, Lutz et al., 2010; Parrott et al., 1996; Wesnes \& Warburton, 1984), or practice on the same day of the session (Harrison et al., 2009; Hendricks et al., 2006). The training session used in this study was similar to a single, 2.5 hour session used in other research (McClernon et al., 2008), though it is plausible that using more training sessions, training to a criterion, or training temporally closer to study sessions may have influenced outcomes. However, it is unclear how the style, duration, and timing of trainings may affect study results. Alternatively, ECIG withdrawal as characterized by cognitive deficits might require a longer period of abstinence. A larger sample size will be needed to determine whether the lack of findings is potentially due to these procedural limitations rather than low statistical power.

ECIG reward. The MCP was used to assess the reward value of ECIGs among this sample of users. The crossover point at which participants chose 10 ECIG puffs over more money was significantly higher at 150 min compared to baseline within the abstinence condition. This same crossover point at $150 \mathrm{~min}$ for abstinence was also higher than at $150 \mathrm{~min}$ for $\mathrm{ad}$ lib use, though the difference was not significant. Studies that have used the MCP to evaluate ECIG reward have primarily done so in cigarette smokers (Maloney et al., 2019; McPherson et al., 2016; Vansickel et al., 2012), or to assess the abuse liability of different ECIG devices in experienced users (Breland et al., 2019). Additionally, purchase tasks for ECIGs may be complicated given that ECIG users often do not quantify use in terms of puffs, and users of different ECIGS may quantify units differently (e.g., cartridges vs. ml of liquid; Cassidy, Tidey, Colby, Long, \& Higgins, 2017). Thus, there is limited research for comparison to findings of the current study. In cigarette smokers, the reward value of cigarettes after overnight abstinence versus no abstinence has been demonstrated using a monetary choice paradigm (Betts \& Tiffany, 
2019) and a variable ratio schedule (Perkins et al., 1994). Results of this study suggest that the MCP may be a useful measure of to evaluate abstinence-induced changes in ECIG reward in future work.

\section{Puff Topography During 180 Minutes of ad lib ECIG Use}

Interrater reliability was high for puff number, puff duration, and IPI, similar to that reported in our previous work that involved video-based measurement of ECIG puff topography during ad lib bouts (Felicione et al., 2019). While others have used video-based measurement and similar operational definitions for topography variables, they have not reported on the reliability and/or validity of this measurement method (Farsalinos et al., 2013a; St. Helen et al., 2016). Puff durations observed in this study $(2.8 \mathrm{sec})$ were higher than $(1.8 \mathrm{sec}$; Lee, Nonnemaker, Bradfield, Hensel, \& Robinson, 2018), lower than (3.5 - 5.9 sec; Farsalinos et al., 2013a; Hiler et al., 2017; Ramôa et al., 2016; Robinson, Hensel, Morabito, \& Roundtree, 2015; Spindle et al., 2017; St. Helen et al., 2016), or similar to (3.0 sec; Kosmider, Jackson, Leigh, O’Connor, \& Goniewicz, 2018) observations reported previously. Differences across studies may be due to differences in the method of measurement used, such as computerized devices in the laboratory (3.5 - 5.9 sec; Hiler et al., 2017; Ramôa et al., 2016; Spindle et al., 2017), computerized devices out of the laboratory (1.8 - $3.5 \mathrm{sec}$; Kosmider, Jackson, et al., 2018; Lee, Nonnemaker et al., 2018; Robinson et al., 2015), or video recording of natural puffing in the lab (3.5 - 4.2 sec; Farsalinos et al., 2013a; St. Helen et al., 2016). They may also be due to differences in the type of ECIG device used: $1^{\text {st }}(1.8-3.5 \mathrm{sec}$; Lee, Nonnemaker et al., 2018; Robinson et al., 2015;), and $2^{\text {nd }}$ and/or $3^{\text {rd }}$ (3.5 - 5.9 sec; Hiler et al., 2017; Ramôa et al., 2016; St Helen et al., 2016; Spindle et al., 2017). In the current study that allowed for natural puffing with 
video-based measurement, the majority of participants used a $4^{\text {th }}$ generation pod style device. No published studies to date have characterized puff topography among users of pod-style ECIGs.

The current study also sought to model the work by St. Helen and colleagues (2016) to characterize clusters of ECIG puffs. This approach may contribute to a determination of definitions for an ECIG-use bout because little is known about how participants puff over long ad lib periods or in their natural environment. Participants in the current study and St. Helen et al. (2016) had different clustering patterns, particularly when considering the percent of total puffs in a session. Participants in the current study had a notably larger percentage of single puffs in the session than participants in the St. Helen study (41.9\% vs $11.9 \%$, respectively), though the percentage of small puff clusters was similar across the studies (49.4\% current study vs $42.9 \%$ St. Helen study). Differences also were observed between studies for medium clusters ( $7.8 \%$ vs $27.8 \%$ ) and large clusters ( $0 \%$ vs $16.5 \%$ ). Despite these differences, the average number of puffs within small and medium clusters was comparable between studies. Additional puff topography differences were found for puff number: 64 puffs in a 90-minutes ad lib session (St Helen et al.) versus 51.4 puffs in 180 minutes in the current study. The longer ad lib session may better represent natural behavior, as an observational study found ECIG users took 156 puffs/day (Kosmider, Jackson et al., 2018). As mentioned earlier, puff durations were shorter in the current study than in the St. Helen study $(2.8 \mathrm{sec}$ vs. $3.5 \mathrm{sec})$, and IPIs in the current study were approximately double that of the St. Helen study (239.2 sec vs. $118 \mathrm{sec})$. The current study added novel data to the literature regarding the average IPI between the end of a cluster and the following puff $(356.4 \mathrm{sec})$ and the average number puffs between clusters (1.7).

Study differences may account for differences in puff topography. Participants in St. Helen et al. (2016) used older ECIG types (tanks vs. pods), lower nicotine concentrations (8.4 
$\mathrm{mg} / \mathrm{ml} \mathrm{vs.} 42.3 \mathrm{mg} / \mathrm{ml}$ ) and were older (38.4 years vs. 20.6 years). Vaping sessions in the St. Helen study were shorter (90 min vs $180 \mathrm{~min}$ ), required a pre-session abstinence period (4 hours vs. no requirement), and did not include administration of tasks or questionnaires, as opposed to multiple subjective and cognitive assessments in the current study. It is not surprising that differences in puff topography were observed between studies given the differences in participant characteristics and study design. However, alternate definitions of puff clustering may be worth consideration in future research, such as IPI length or the amount of puffs in small, medium, and large clusters. Observational studies have made participants define sessions by turning a topography measurement device on and off (Kosmider, Jackson, et al., 2018; Lee, Nonnemaker et al., 2018), and found that ECIG users took 10 - 12 puffs/session with an IPI of $21.7 \mathrm{sec}$, though the amount of sessions differed between studies $(3.5-15.3)$. This may suggest that how participants cluster puffs outside of the laboratory may differ from clustering in laboratory ad lib sessions, however, these studies involved the use of computerized topography devices that may influence puffing patterns (e.g., more sessions, no individual puffs). Continued research to define ECIG bouts can greatly benefit characterization of ECIG puff topography.

Individual differences should be considered when assessing ECIG puff topography. Large variability in puffing patterns can be observed in Figures 14 - 16, in which participant 501 had almost all puffs in clusters $(95.4 \%)$, participant 507 had few clustered puffs $(11.8 \%)$ and participant 513 had a moderate amount of clustered puffs (44.0\%). Similarly, large variability in the percentage of single puffs in a session was observed in the current study $(0.0-88.2 \%)$ and by St. Helen et al. $(0.0-42.3 \%)$. Wide ranges were also observed for puff number $(17-77)$, average puff duration $(1.1 \mathrm{~s}-4.6 \mathrm{sec})$ and average IPI (137.4 s $-468.8 \mathrm{sec})$. Some previous research has characterized puff topography with an emphasis on individual subjects due to 
differences in ECIG puffing patterns (Robinson et al., 2015; Robinson et al., 2016; St. Helen et al., 2016), and has split participants into groups based on puffing patterns (Robinson et al., 2015) or defined different types of vaping sessions (Lee, Morgan-Lopez et al., 2018). It likely will be useful to acknowledge different puffing patterns among ECIG users as researchers characterize ECIG bouts, as a standard definition may not be applicable to all ECIG users.

\section{Predicting Withdrawal from Puff Topography}

An exploratory outcome of the study was the prediction of withdrawal based on puffing topography. Topography significantly predicted $38.5 \%$ of subjective withdrawal outcomes and $42.1 \%$ of nicotine/product effects. It was hypothesized that more puffs and longer durations, would be associated with withdrawal, though the opposite pattern was found. For example, longer puff durations and fewer puff clusters were associated with reduced heart rate. Longer puff durations were commonly associated with higher ratings of subjective withdrawal (e.g., craving, hunger, impatient) and lower product effects (e.g., concentrate, reduce hunger). The strongest predictive effects were found for impatient, in which puff topography accounted for $42 \%$ of the variance. However, the majority of these regressions accounted for less than $20 \%$ of the outcome variance, giving these analyses limited predictive validity.

Results of these models are complicated to interpret due to third variables that may influence findings. Those that were more dependent may have used ECIGs more intensively and also experienced more severe withdrawal outcomes. An additional concern is the lack of control in long ad lib vaping sessions that may introduce other factors (e.g., boredom, cues) that can influence behavior and withdrawal. Similarly, ad lib vaping allows all vaping parameters (puff number, duration, IPI) to vary, as opposed to standardized puffing bouts that hold certain variables constant while others vary. These analyses would benefit from the use of a 
computerized topography measurement device that can capture variables such as puff volume and flow rate. Finally, puff number and puff clusters were correlated, which potentially introduced issues of multicollinearity into regressions.

\section{Conclusions}

There are several notable strengths of the current study. The use of an abstinence manipulation allows for the comparison between $a d$ lib vaping and vaping abstinence, along with comparisons between baseline abstinence and increasing durations of abstinence. Including predeprived and nondeprived assessments gives strength to conclusions about abstinenceinduced deficits, rather than nicotine enhancement of performance (Heishman et al., 1994). However, future research with a non-smoking control group would allow for more definitive conclusions on this matter. Future research may also wish to include a $0 \mathrm{mg} / \mathrm{ml}$ nicotine condition (placebo-control), though the exclusion of this condition strengthens the ecological validity of the current study. The exclusion of regular cigarette smokers allows for conclusions regarding dependence and withdrawal in ECIG users that are not currently or formerly dependent upon cigarettes. The use of participants' usual ECIGs in the ad lib condition created a more naturalistic representation of normal behavior than a standardized ECIG. Use of a participants' usual ECIGs likely allows participants to puff more naturally and experience typical levels of nicotine exposure, which can have important implications for cognitive and subjective assessments. The current study includes measurement techniques and analyses that have been common in the cigarette dependence and withdrawal literature, but not ECIG literature. For example, little is known about cognitive performance in ECIG users under abstinent or ad lib conditions. Additionally, little work has been done to assess diagnoses of tobacco use disorder in 
ECIG users, and few studies have assessed puff topography during long ad lib use bouts without a computerized topography device.

Future research should expand upon findings of the current study. The current study included a small sample size and was underpowered to detect small to moderate effect sizes. Increasing the sample size would provide important information regarding which effects exist but were underpowered in the current study versus which withdrawal symptoms may not be experienced by ECIG users. Additionally, the current sample may have limited generalizability to a larger population of ECIG users. Though ECIG use among nonsmokers is highest in young adults (Schoenborn \& Gindi, 2016), adolescent and older adult ECIG users may differ in use patterns and dependence from young adult users. Similarly, most participants in the current sample used $4^{\text {th }}$ generation pod devices. These devices may have nicotine delivery profiles different from that of other ECIGs, which may influence withdrawal and puff topography. The current study also sought out individuals with higher ECIG dependence scores, and thus these participants may have experienced greater withdrawal than the average ECIG user. A longer abstinence manipulation would help further characterize the timecourse of ECIG withdrawal and determine if certain effects, particularly cognitive effects, are experienced later than two hours of abstinence. While studies of cigarette smokers have shown abstinence-induced effects within two hours (Hendricks et al., 2006; Parrott \& Craig, 1996), other studies have shown less reliable effects at early hours of abstinence (Hatsukami et al., 1989; Heimstra, Fallesen, Kinsley, \& Warner, 1980). Assessing the effects of overnight abstinence on cognitive performance is an essential next step for characterizing ECIG withdrawal. Currently, the only method to verify short-term ECIG abstinence is to test plasma nicotine concentrations or observe users in the lab. Testing plasma nicotine concentrations is not always feasible, making alternate biochemical tests 
of ECIG abstinence of great utility to researchers. An inpatient study in which ECIG users are held overnight to confirm abstinence would help define the effects of a longer abstinence duration until such tests are available. Additional future directions may involve the comparison of ECIG users of different device types. The type of ECIG used likely influences puff topography, nicotine delivery (Wagener et al., 2017), and may affect subjective responses and cognitive performance. While the current study involved mostly $4^{\text {th }}$ generation pod users and some $3^{\text {rd }}$ generation mod users, the sample size was not large enough to characterize differences between these groups. Additional considerations may include widening inclusion criteria to include participants that score lower on ECIG dependence. As mentioned previously, the current study purposely sought out those higher in ECIG dependence, and thus, the findings likely are not generalizable to a larger population of ECIG users.

Despite the limitations, the current study is novel in that it a) assessed the effects of shortterm abstinence on ECIG withdrawal symptoms and b) assessed puff topography, subjective reports, cognitive performance, ECIG reward, and dependence measures in ECIG users that are not cigarette smokers. Generally, increases in subjective withdrawal symptoms and reductions in heart rate and nicotine effects were observed in the abstinence condition, with onset of effects within two hours of abstinence. Mixed results were observed for cognitive tasks, with the clearest abstinence-induced deficits observed for response inhibition. Findings of the study must be interpreted with consideration to limited power and generalizability; however, it provides evidence of the early onset of ECIG withdrawal, indicates that non-smoking ECIG users are nicotine dependent, and sets a foundation for research to continue studying these effects in the future. 


\section{References}

American Psychiatric Association. (2013). Diagnostic and statistical manual of mental disorders (5 $5^{\text {th }}$ ed.). Washington, DC: American Psychiatric Association.

Ashare, R. L., Falcone, M., \& Lerman, C. (2014). Cognitive function during withdrawal: Implications for nicotine dependence treatment. Neuropharmacology, 76, 581-591. doi:10.1016/j.neuropharm.2013.04.034

Ashare, R. L., \& Hawk, L. W. Jr., (2014). Effects of smoking abstinence on impulsive behavior among smokers high and low in ADHD-like symptoms. Psychopharmacology, 219(2), 537-547. doi:10.1007/s00213-011-2324-2

Atzori, G., Lemmonds, C. A., Kotler, M. L., Durcan, M. J., \& Boyle, J. (2008). Efficacy of a nicotine (4 mg)-containing lozenge on the cognitive impairment of nicotine withdrawal. Journal of Clinical Psychopharmacology, 28, 667-674.

Audrain-McGovern, J., Stone, M. D., Barrington-Trimis, J., Unger, J. B., \& Leventhal, A. (2018). Adolescent e-cigarette, hookah, and conventional cigarette use and subsequent marijuana use. Pediatrics, 142, e20173616. doi:10.1542/peds.2017-3616

Ayers, J. W., Ribisl, K. M., Brownstein, J. S. (2011). Tracking the ride in the popularity of electronic nicotine delivery systems (electronic cigarettes) using search query surveillance. American Journal of Preventative Medicine, 40(4), 448-453. doi:10.1016/j.amepre.2010.12.007

Baddeley, A. (1992). Working Memory. Science, 255, 556-559.

Baddeley, A. D., \& Hitch, G. J. (1994). Developments in the concept of working memory. Neuropsychology, 8(4), 485-493. 
Baker, T. B., Breslau, N., Covey, L., \& Shiffman, S., (2012). DSM criteria for tobacco use disorder and tobacco withdrawal: A critique and proposed revisions for DSM-5. Addiction, 107(2), 263-275. doi:10.1111/j.1360-0443.2011.03657.x

Benjamini, Y., \& Hochberg, Y. (1995). Controlling the false discovery rate: A practical and powerful approach to multiple testing. Journal of the Royal Statistical Society. Series B (Methodological), 57, 289-300.

Benowitz, N. L. (2010). Nicotine Addiction. New England Journal of Medicine, 362(24), 22952303. doi:10.1056/NEJMra0809890

Betts, J. M., \& Tiffany, S. T. (2019). Comparing the reward value of cigarettes and food during tobacco abstinence and nonabstinence. Drug and Alcohol Dependence, 204, 107475. doi:10.1016/j.drugalcdep.2019.04.040

Blank, M. D., Breland, A. B., Cobb, C. O., Spindle, T., Ramôa, C., \& Eissenberg, T. (2016). Clinical laboratory evaluation of electronic cigarettes: Methodological challenges. Tobacco Regulatory Science, 2(4), 426-439. doi:10.18001/TRS.2.4.12

Blank, M. D., Disharoon, S., \& Eissenberg, T. (2009). Comparison of methods for measurement of smoking behavior: mouthpiece-based computerized devices versus direct observation. Nicotine \& Tobacco Research, 11(7), 896-903. doi:10.1093/ntr/ntp083

Blank, M. D., Pearson, J., Cobb, C. O., Felicione, N. J., Hiler, M., Spindle, T. R., \& Breland, A. B. (in press). What individual-level factors predict electronic cigarette nicotine delivery? Tobacco Control.

Brandon, T. H., Goniewicz, M. L., Hanna, N. H., Hatsukami, D. K., Herbst, R. S., Hobin, J. A., . . Warren, G. W. (2015). Electronic nicotine delivery systems: A policy statement from 
the American Association for Cancer Research and the American Society of Clinical Oncology. Journal of Clinical Oncology, 33, 952-963. doi:10.1200/JCO.2014.59.4465

Braver, T. S., Barch, D. M., Keys, B. A., Carter, C. S., Cohen, J. D., Kaye, J. A., . . . Mumenthaler, M. S. (2001). Context processing in older adults: Evidence for a theory relating cognitive control to neurobiology in healthy aging. Journal of Experimental Psychology: General, 130(4), 746-763. doi:10.1037//0096-3445.130.4.746

Breland, A. B., Buchhalter, A. R., Evans, S. E., \& Eissenberg, T. (2002). Evaluating acute effects of potential reduced-exposure products for smokers: clinical laboratory methodology. Nicotine \& Tobacco Research, S131-S140. doi:10.1080/1462220021000032780

Breland, A. B., Kleykamp, B. A., \& Eissenberg, T. (2006). Clinical laboratory evaluation of potential reduced exposure products for smokers. Nicotine \& Tobacco Research, 8(6), 727-738. doi:10.1080/14622200600789585

Breland, A. B., Maloney, S. F., Soule, E. K., Ramôa, A. C., Barnes, A., Lipato, T., \& Eissenberg, T. (2019). Abuse liability of electronic cigarettes in men who are experienced electronic cigarette users. Experimental and Clinical Psychopharmacology. Epub ahead of print. doi: $10.1037 /$ pha0000305

Brown, C. J., \& Cheng, J. M. (2014). Electronic cigarettes: product characterization and design considerations. Tobacco Control, 23, ii4-ii10. doi:10.1136/tobaccocontrol-2013-051476

Browne, M., \& Todd, D. G. (2018). Then and now: Consumption and dependence in e-cigarette users who formerly smoked cigarettes. Addictive Behaviors, 76, 113-121. doi:10.1016/j.addbeh.2017.07.034 
Buchhalter, A. R., \& Eissenberg, T. (2000). Preliminary evaluation of a novel smoking system: Effects on subjective and physiological measures and on smoking behavior. Nicotine \& Tobacco Research, 2, 39-43.

Bullen, C., McRobbie, H., Thornley, S., Glover, M., Lin, R., \& Laugesen, M. (2010). Effect of an electronic nicotine delivery device (e cigarette) on desire to smoke and withdrawal, user preferences and nicotine delivery: Randomised cross-over trial. Tobacco Control, 19(2), 98-103. doi:10.1136/tc.2009.031567

Cassidy, R. N., Tidey, J. W., Colby, S. M., Long, V. \& Higgins, S. T. (2017). Initial development of an e-cigarette purchase task: A mixed methods study. Tobacco Regulatory Science, 3, 139-150. doi:10.18001/TRS.3.2.2

Cobb, C. O., Shihadeh, A., Weaver, M. F., \& Eissenberg, T. (2011). Waterpipe tobacco smoking and cigarette smoking: A direct comparison of toxicant exposure and subjective effects. Nicotine \& Tobacco Research, 13(2), 78-87. doi:10.1093/ntr/ntq212

Cohen, J. (1988). Statistical power analysis for the behavior ( $2^{\text {nd }}$ ed). Orlando, FL: Academic Press.

Cook, M. R., Gerkovich, M. M., Graham, C., Hoffman, S. J., \& Peterson, R. C. (2003). Effects of the nicotine patch on performance during the first week of smoking cessation. Nicotine \& Tobacco Research, 5, 169-180.

Cox, L. S., Tiffany, S. T., \& Christen, A. G. (2001). Evaluation of the brief questionnaire of smoking urges (QSU-brief) in laboratory and clinical settings. Nicotine \& Tobacco Research, 3, 7-16. 
Crean, R. D., Crane, N. A., \& Mason, B. J. (2011). Evidence based review of acute and longterm effects of cannabis use on executive cognitive functions. Journal of Addiction Medicine, 5, 1-8. doi:10.1097/ADM.0b013e31820c23fa

Dalessandro, C. (2018). Recruitment tools for reaching millennials: The digital difference. International Journal of Qualitative Methods. doi:10.1177/1609406918774446.

Davidson, M. M., Cronk, N. J., Harris, K. J., Harrar, S., Catley, D., \& Good, G. E. (2010). Strategies to recruit and retain college smokers in cessation trials. Research in Nursing \& Health, 33, 144-155. doi:10.1002/nur.20372

Dawkins, L., \& Corcoran, O. (2014). Acute electronic cigarette use: Nicotine delivery and subjective effects in regular users. Psychopharmacology, 231, 401-407. doi:10.1007/s00213-013-3249-8

Dawkins, L., Turner, J., Hasna, S., \& Soar, K. (2012). The electronic-cigarette: Effects on desire to smoke, withdrawal symptoms and cognition. Addictive Behaviors, 37, 970-973. doi:10.1016/j.addbeh.2012.03.004

Diamond, A. (2013). Executive Functions. Annual Review of Psychology, 64, 135-168. doi:10.1146/annurev-psych-113011-143750

Eissenberg, T., Soule, E., Saliba, N., et al. (2018, June). JUUL: the prototypical "pod mod”: Design characteristics, toxicant yield, and preliminary nicotine delivery and subjective effect profile. Presented at National Institutes of Health (NIH) Tobacco Regulatory Science Meeting, Bethesda, MD.

Ernst, M., Heishman, S. J., Spurgeon, L., \& London, E. D. (2001). Smoking history and nicotine effects on cognitive performance. Neuropsychopharmacology, 25(3), 313-319. 
Etter, J. F. (2005). A self-administered questionnaire to measure cigarette withdrawal symptoms, the Cigarette Withdrawal Scale. Nicotine \& Tobacco Research, 7, 47-57.

Etter, J. F., \& Eissenberg, T. (2015). Dependence levels in users of electronic cigarettes, nicotine gums, and tobacco cigarettes. Drug and Alcohol Dependence, 147, 68-75. doi:10.1016/j.drugalcdep.2014.12.007

Etter, J. F., \& Hughes, J. R. (2006). A comparison of the psychometric properties of three cigarette withdrawal scales. Addiction, 101, 362-372.

doi:10.1111/j.1360-0443.2005.01289.x

Evans, D. E., \& Drobes, D. J. (2009). Nicotine self-medication of cognitive-attentional processing. Addiction Biology, 14, 32-42. doi:10.1111/j.1369-1600.2008.0013.x

Evans, S. E., Blank, M., Sams, C., Weaver, M. F., \& Eissenberg, T. (2006). Transdermal nicotine-induced tobacco abstinence symptom suppression: Nicotine dose and smokers' gender. Experimental and Clinical Psychopharmacology, 14, 121-135.

Fagerström, K., \& Eissenberg, T. (2012). Dependence on tobacco and nicotine products: A case for product-specific assessment. Nicotine \& Tobacco Research, 14(11), 1382-1390.

Falcone, M., Wileyto, E. P., Ruparel, K., Gerraty, R. T., Laprate, L., Detre, J. A., . . Lerman, C. (2014). Age-related differences in working memory deficits during nicotine withdrawal. Addiction Biology, 19, 907-917. doi:10.1111/adb.12051

Farsalinos, K. E., Romagna, G., Tsiapras, D., Kyrzopoulos, S., Voudris, V. (2013a). Evaluation of electronic cigarette user (vaping) topography and estimation of liquid consumption: implications for research protocol standard definition and for public health authorities' regulation. International Journal of Environmental Research and Public Health, 10, 2500-2514. doi:10.3390/ijerph10062500 
Farsalinos, K. E., Romagna, G., Tsiapras, D., Kyrzopoulos, S., \& Voudris, V. (2013b).

Evaluating nicotine levels selection and patterns of cigarette use in a group of "vapers" who had achieved complete substitution of smoking. Substance Abuse: Research and Treatment, 7, 139-146. doi:10.4137/SART.S12756

Farsalinos, K. E., Spyrou, A., Tsimopoulou, K., Stefopoulos, C., Romagna, G., \& Voudris, V. (2014). Nicotine absorption from electronic cigarette use: Comparison between first and new-generation devices. Scientific Reports, 4, 4133. doi:10.1038/srep04133

Felicione, N., Ozga-Hess, O., Karaoghlanian, N., Shihadeh, A., Eissenberg, T., Haliwa, I., .. . Blank, M. (2019, February). Comparison of measurement methods for electronic cigarette topography. Poster presented at $25^{\text {th }}$ annual meeting of the Society for Research on Nicotine and Tobacco, San Francisco, CA.

Ferguson, S. G., Shiffman, S., \& Gwaltney, C. J. (2006). Does reducing withdrawal severity mediate nicotine patch efficacy? A randomized clinical trial. Journal of Consulting and Clinical Psychology, 74(6), 1153-1161. doi:10.1037/0022-006X.74.6.1153

Foulds, J., Stapleton, J., Swettenham, J., Bell, N., McSorely, K., \& Russell, M.A. (1996). Cognitive performance effects of subcutaneous nicotine in smokers and never-smokers. Psychopharmacology, 127, 31-38.

Foulds, J., Veldheer, S., Yingst, J., Hrabovsky, S., Wilson, S. J., Nichols, T. T., \& Eissenberg, T. (2015). Development of a questionnaire of assessing dependence of electronic cigarettes among a large sample of ex-smoking e-cigarette users. Nicotine \& Tobacco Research, 17(2), 186-192. doi:10.1093/ntr/ntu204

Gilbert, D. G., Izetelny, A., Radtke, R., Hammersley, J., Rabinovich, N. E., Jameson, T. R., \& Huggenvik, J. I. (2005). Dopamine receptor (drd2) genotype-dependent effects of 
nicotine on attention and distraction during rapid visual information processing. Nicotine \& Tobacco Research, 7(3), 361-370. doi:10.1080/14622200500125245

Goniewicz, M. L., Boykan, R., Messiina, C. R., Eliscu, A., \& Tolentino, J. (2018). High exposure to nicotine among adolescents who use Juul and other vape pod systems. Tobacco Control. Epub ahead of print. doi:10.1136/tobaccocontrol-2018-054565

Gray, J. N., Breland, A. B., Weaver, M., \& Eissenberg, T. (2008). Potential reduced exposure products (PREPs) for smokeless tobacco users: Clinical evaluation methodology. Nicotine \& Tobacco Research, 10(9), 1441-1448. doi:10.1080/14622200802323258

Griffiths, R. R., Rush, C. R., \& Puhala, K. A. (1996). Validation of the multiple-choice procedure for investigating drug reinforcement in humans. Experimental and Clinical Psychopharmacology, 4, 97-106.

Griffiths, R. R., Triosi II, J. R., Silverman, K., \& Mumford, G. K. (1993). Multiple-choice procedure: An efficient approach for investigating drug reinforcement in humans. Behavioural Pharmacology, 4, 3-13.

Grundey, J., Amu, R., Gergely Ambrus, G., Batsikadze, G., Paulus, W., \& Nitsche, M. A. (2015). Double dissociation of working memory and attentional processes in smokers and non-smokers with and without nicotine. Psychopharmacology, 232, 2491-2501. doi:10.1007/s00213-015-3880-7

Harrison, E. L. R., Coppola, S., \& McKee, S. A. (2009). Nicotine deprivation and trait impulsivity affect smokers' performance on cognitive tasks of inhibition and attention. Experimental and Clinical Psychopharmacology, 17(2), 91-98. doi:10.1037/a0015657

Haste, H. (2005). Joined-up texting: Mobile phones and young people. Young Consumers, 6, 5667. doi:10.1108/17473610510701214 
Hatsukami, D., Fletcher, L., Morgan, S., Keenan, R., \& Amble, P. (1989). The effects of varying cigarette deprivation duration on cognitive and performance tasks. Journal of Substance Abuse, 1, 407-416.

Heimstra, N. W., Fallesen, J. J., Kinsley, S. A., \& Warner, N. W. (1980). The effects of deprivation of cigarette smoking on psychomotor performance. Ergonomics, 23, 10471055.

Heishman, S. J., Taylor, R. C., \& Henningfield, J. E. (1994). Nicotine and smoking: A review of effects on human performance. Experimental and Clinical Psychopharmacology, 2(4), 345-395.

Hemmerich, N., Klein, E. G., \& Berman, M. (2017). Evidentiary support in public comments to the FDA's center for tobacco products. Journal of Health Politics, Policy and Law, 42(4), 645-666. doi:10.1215/03616878-3856121

Hendricks, P. S., Ditre, J. W., Drobes, D. J., \& Brandon, T. H. (2006). The early time course of smoking withdrawal effects. Psychopharmacology, 187, 385-396.

doi:10.1007/s200213-006-0429-9

Henningfield, J. E., \& Keenan, R. M. (1993). Nicotine delivery kinetics and abuse liability. Journal of Consulting and Clinical Psychology, 61(5), 743-750.

Hensel, E. C., Eddingsaas, N. C., DiFrancesco, A. G., Jayasekera, S., O’Dea, S., \& Robinson, R. J. (2019). Framework to estimate total particulate mass and nicotine delivered to e-cig users from natural environment monitoring data. Scientific Reports, 9, 8572. doi:10.1038/s41598-019-44983-w

Herbert, M., Foulds, J., \& Fife-Schaw, C. (2001). No effect of cigarette smoking on attention or mood in non-deprived smokers. Addiction, 96, 1349-1356. 
Hiler, M., Breland, A., Spindle, T., Maloney, S., Lipato, T., Karaoghlanian, ... Eissenberg, T. (2017). Electronic cigarette user plasma nicotine concentration, puff topography, heart rate, and subjective effects: Influence of liquid nicotine concentration and user experience. Experimental and Clinical Psychopharmacology, 25(5), 380-392. doi: $10.1037 /$ pha0000140

Hu, P., Fan, T., Yingst, J., Veldheer, S., Hrabovsky, S., Chen, C. \& Fould,s J. (2019). Changes in e-cigarette use behaviors and dependence in long-term e-cigarette users. American Journal of Preventive Medicine, 57, 374-383. doi:10.1016/j.amepre.2019.04.021

Huang, J., Duan, Z., Kwok, J., Binns, S., Vera, L. E., Kim, Y., . . . Emery, S. L. (2019). Vaping versus JUULing: How the extraordinary growth and marketing of JUUL transformed the US retail e-cigarette market. Tobacco Control, 28, 146-151. doi:10.1136/tobaccocontrol-2018-054382

Hughes, J. R. (2007a). Effects of abstinence from tobacco: Valid symptoms and time course. Nicotine \& Tobacco Research, 9(3), 315-327. doi:10.1080/14622200701188919

Hughes, J. R. (2007b). Measurement of the effects of abstinence from tobacco: A qualitative review. Psychology of Addictive Behaviors, 21(2), 127-137. doi:10.1037/0893-164X.21.2.127

Hughes, J. R., \& Callas, P. W. (2019). Prevalence of withdrawal symptoms from electronic cigarette cessation: A cross-sectional analysis of US Population Assessment of Tobacco and Health. Addictive Behaviors, 91, 234-237. doi:10.1016/j.addbeh.2019.07.002

Hughes, J. R., \& Hatsukami, D. (1986). Signs and symptoms of tobacco withdrawal. Archives of General Psychiatry, 43, $289-294$. 
Hughes, J. R., Peters, E. N., Callas, P. W., Peasley-Miklus, C., Oga, E., Etter, J. F., \& Morley, N. (2019a). Withdrawal symptoms from e-cigarette abstinence among former smokers: A pre-post clinical trial. Nicotine \& Tobacco Research. Epub ahead of print. doi:10.1093/ntr/ntz129

Hughes, J. R., Peters, E. N., Callas, P. W., Peasley-Miklus, C., Oga, E., Etter, J. F., \& Morley, N. (2019b). Withdrawal symptoms among e-cigarette abstinence among adult neversmokers: A pilot experimental study. Nicotine and Tobacco Research. Epub ahead of print. doi:10.1093/ntr/ntz169

Huynh, H., \& Feldt, L.S. (1976). Estimation of the box correction for degrees of freedom from sample data in randomized block and split-plot designs. Journal of Educational and Behavioral Statistics, 1(1), 69-82. doi:10.3102/10769986001001069

Ickes, M., Hester, J. W., Wiggins, A. T., Rayens, M. K., Hahn, E. J., \& Kavuluru, R. (2019). Prevalence and reasons for Juul use among college students. Journal of American College Health. Epub ahead of print. doi:10.1080/07448481.2019.1566867

Jacobsen, L. K., Krystal, J. H., Mencl, W. E., Westerveld, M., Frost, S. J., \& Pugh, K. R. (2005). Effects of smoking and smoking abstinence on cognition in adolescent tobacco smokers. Biological Psychiatry, 57, 56-66.

Jacobsen, L. K., Pugh, K. R., Constable, R. T., Westerveld, M., \& Mencl, W. E. (2007). Functional correlates of verbal memory deficits emerging during nicotine withdrawal in abstinent adolescent cannabis users. Biological Psychiatry, 61, 31-40.

Johnson, J. M., Muilenburg, J. L., Rathbun, S. L., Yu, X., Naeher, L. P., \& Wang, J. S. (2018). Elevated nicotine dependence scores among electronic cigarette users at an electronic cigarette convention. Journal of Community Health, 43, 164-174. 
doi:10.1007/s10900-017-0399-3

Jolma, C. D., Samson, R. A., Klewer, S. E., Donnerstein, R. L., \& Goldberg, S. J. (2002). Acute cardiac effects of nicotine in healthy young adults. Echocardiography, 19, 443-448.

Jonides, J., Schumacher, E. H., Smith, E. E., Lauber, E. J., Awh, E., Minoshima, S., \& Koeppe, R. A. (1997). Verbal working memory load affects regional brain activation as measured by PET. Journal of Cognitive Neuroscience, 9, 462-475.

Keppel, G. (1991). Design and analysis: A researcher's handbook. Englewood Cliffs, NJ: Prentice Hall.

Khatamian Far, P. (2018). Challenges of recruitment and retention of university students as research participants: Lessons learned from a pilot study. Journal of the Australian Library and Information Association, 67, 278-292. doi:10.1080/24750158.2018.1500436

Kim, H. Y. (2013). Statistical notes for clinical researchers: Assessing normal distribution (2) using skewness and kurtosis. Restorative Dentistry \& Endodontics, 38, 52-54. doi:10.5395/red.2013.38.1.52

King, B. A., Patel, R., Nguyen, K. H., \& Dube, S. R. (2015). Trends in awareness and use of electronic cigarettes among US adults, 2010-2013. Nicotine \& Tobacco Research. 17(2), 219-227. doi:10.1093/ntr/ntu191

Kleykamp, B. A., Jennings, J. M., Blank, M. D., \& Eissenberg, T. (2005). The effects of nicotine on attention and working memory in never-smokers. Psychology of Addictive Behaviors, 19(4), 433-438. doi:10.1037/0893-164X.19.4.433

Kleykamp, B. A., Jennings, J. M., \& Eissenberg, T. (2011). Effects of transdermal nicotine and concurrent smoking on cognitive performance in tobacco-abstinent smokers. Experimental and Clinical Psychopharmacology, 19, 75-84. doi:10.1037/a0022417 
Knudsen, E. I. (2007). Fundamental components of attention. Annual Review of Neuroscience, 30, 57-78. doi:10.1146/annurev.neuro.30.051606.094256

Kosmider, L., Jackson, A., Leigh, N., O’Connor, R., \& Goniewicz, M. L. (2018). Circadian puffing behavior and topography among e-cigarette users. Tobacco Regulatory Science, 4, 41-49. doi:10.18001/TRS.4.5.4

Kosmider, L., Sobczak, A., Knysak, J., \& Goniewicz, M.L. (2014). Effects of solvent and battery output voltage on nicotine levels released from electronic cigarettes. Presented at the 20th annual meeting of the Society for Research on Nicotine and Tobacco, Seattle, WA, February.

Kosmider, L., Spindle, T. R., Gawron, M., Sobczak, A., Goniewicz, M. L. (2018). Nicotine emissions from electronic cigarettes: Individual and interactive effect of propylene glycol to vegetable glycerin composition and device power output. Food and Chemical Toxicology, 115, 302-305. doi:10.1016/j.fct.2019.03.025

Kozink, R. V., Kollins, S. H., \& McClernon, F. J. (2010). Smoking withdrawal modulates right inferior frontal cortex but not presupplementary motor activation during inhibitory control. Neuropsychopharmacology, 35, 2600-2606. doi:10.1038/npp.2010.154

Kozink, R. V., Lutz, A. M., Rose, J. E., Froeliger, B., \& McClernon, F. K. (2010b). Smoking withdrawal shifts the spatiotemporal dynamics of neurocognition. Addiction Biology, 15(4), 480-490. doi:10.1111/j.1369-1600.2010.00252.x

Lanza, S. T., \& Vasilenko, S. A. (2015). New methods shed light on age of onset as a risk factor for nicotine dependence. Addictive Behaviors, 50, 161-164.

doi:10.1016/j.addbeh.2015.06.024 
Le Houezec, J. (2003). Role of nicotine pharmacokinetics in nicotine addiction and nicotine replacement therapy: A review. International Journal of Tuberculosis and Lung Disease, $7(9), 811-819$.

Leavens, E. L. S., Stevens, E. M., Brett, E. I., \& Leffingwell, T. R. (2019). JUUL in school: JUUL electronic cigarette use patterns, reasons for use, and social normative perceptions among college student ever users. Addictive Behaviors, 99, 106047. doi:10.1016/j.addbeh.2019.106047

Lee, Y. O., Morgan-Lopez, A. A., Nonnemaker, J. M., Pepper, J. K., Hensel, E. C., \& Robinson, R. J. (2018). Latent class analysis of e-cigarette use sessions in their natural environment. Nicotine \& Tobacco Research. Epub ahead of print. doi:10.1093/ntr/nty164

Lee, Y. O., Nonnemaker, J. M., Bradfield, B., Hensel, E. C., \& Robinson, R. J. (2018). Examining daily electronic cigarette puff topography among established and nonestablished cigarette smokers in their natural environment. Nicotine \& Tobacco Research, 20, 1283-1288. doi:10.1093/ntr/ntx222

Leventhal, A. M., Waters, A. J., Moolchan, E. T., Heishman, S. J., \& Pickworth, W. B. (2010). A quantitative analysis of subjective, cognitive, and physiological manifestations of the acute tobacco abstinence syndrome. Addictive Behaviors, 35(12), 1120-1130. doi:10.1016/j.addbeh.2010.08.007

Maloney, S. F., Breland, A., Soule, E. K., Hiler, M., Ramôa, C., Lipato, T., \& Eissenberg, T. (2019). Abuse liability assessment of an electronic cigarette in combustible cigarette smokers. Experimental and Clinical Psychopharmacology. Epub ahead of print. doi: $10.1037 /$ pha0000261 
Mancuso, G., Andres, P., Ansseau, M., \& Tirelli, E. (1999). Effects of nicotine administered via a transdermal delivery system of vigilance: A repeated measure study. Psychopharmacology, 142, 18-23.

Marczinski, C. A., \& Fillmore, M. T. (2003). Dissociative antagonistic effects of caffeine on alcohol-induced impairment of behavioral control. Experimental and Clinical Psychopharmacology, 11(3), 228-236. doi:10.1037/1064-1297.11.3.228

Maziak, W., Rastam, S., Ibrahim, I., Ward, K. D., Shihadeh, A., \& Eissenberg, T. (2009). CO exposure, puff topography, and subjective effects in waterpipe tobacco smokers. Nicotine \& Tobacco Research, 11(7), 806-811. doi:10.1093/ntr/ntp066

McClernon, F. J., Addicott, M. A., \& Sweitzer, M. M. (2015). Smoking abstinence and neurocognition: Implications for cessation and relapse. In The Neurobiology and Genetics of Nicotine and Tobacco (pp. 193-227). Switzerland: Springer International Publishing.

McClernon, F. J., Kollins, S. H., Lutz, A. M., Fitzgerald, D. P., Murray, D. W., Redman, C., \& Rose, J. E. (2008). Effects of smoking abstinence on adult smokers with and without attention deficit hyperactive disorder: Results of a preliminary study. Psychopharmacology, 197, 95-105. doi:10.1007/s00213-007-1009-3

McMillen, R. C., Gottlieb, M. A., Shaefer, R. M., Winickoff, J. P., \& Klein, J. D. (2015). Trends in electronic cigarette use among U.S. adults: Use is increasing in both smokers and nonsmokers. Nicotine \& Tobacco Research, 17, 1195-1202. doi:10.1093/ntr/ntu213

McPherson, S., Howell, D., Lewis, J., Barbosa-Leiker, C., Bertotti Metoyer, P., \& Roll, J. (2016). Self-reported smoking effects and comparative value between cigarettes and high 
dose e-cigarettes in nicotine-dependent cigarette smokers. Behavioural Pharmacology, 27, 301-307. doi:10.1097/FBP.0000000000000226

Mendrek, A., Monterosso, J., Simon, S. L., Jarvik, M., Brody, A., Olmstead, R., . . London, E. D. (2006). Working memory in cigarette smokers: Comparison to non-smokers and effects of abstinence. Addictive Behaviors, 31(5), 833-844. doi:10.1016/j.addbeh.2005.06.009

Mirbolouk, M., Charkhchi, P., Kianoush, S., Uddin, I., Orimoloye, O. A., Jaber, R., ... Blaha, M. J. (2018). Prevalence and distribution of e-cigarette use among U.S. adults: Behavioral risk factor surveillance system, 2016. Annals of Internal Medicine, 169, 429438. doi:10.7326/M17-3440

Molander, L., Lunell, E., \& Fagerström, K. O. (2000). Reduction of tobacco withdrawal symptoms with a sublingual nicotine tablet: A placebo controlled study. Nicotine \& Tobacco Research, 2, 187-191. doi:10.1080/713688123

Morean, M. E., Krishnan-Sarin, S., \& O’Malley, S. S. (2018). Comparing cigarette and ecigarette dependence and predicting frequency of smoking and e-cigarette use in dualusers of cigarettes and e-cigarettes. Addictive Behaviors, 87, 92-96. doi:10.1016/j.addbeh.2018.06.027

Morean, M. E., Krishnan-Sarin, S., Sussman, S., Foulds, J. Fishbein, H., Grana, R., \& O’Malley, S. S. (2019). Psychometric evaluation of the Patient-Reported Outcomes Measurement Information System (PROMIS) Nicotine Dependence item back for use with electronic cigarettes. Nicotine \& Tobacco Research. Epub ahead of print. doi:10.1093/ntr/ntz095 
Muramoto, M. L., Ranger-Moore, J., \& Leischow, S. J. (2003). Efficacy of oral transmucosal nicotine lozenge for suppression of withdrawal symptoms in smoking abstinence. Nicotine 7 Tobacco Research, 5(2), 223-230.

Myers, C. S., Taylor, R. C., Moolchan, E. T., \& Heishman, S. J. (2008). Dose-related enhancement of mood and cognition in smokers administered nicotine nasal spray. Neuropsychopharmacology, 33, 588-598. doi:10.1038/sj.npp.1301425

Norton, K. J., June, K. M., \& O’Connor, R. J. (2014). Initial puffing behaviors and subjective Reponses differ between an electronic nicotine delivery system and traditional cigarettes. Tobacco Induced Diseases, 12, 17. doi:10.1186/1617-9625-12-17

O’Brien, R. M. (2007). A caution regarding rules of thumb for variance inflation factors. Quality \& Quantity, 41, 673-690. doi:10.1007/s11135-006-9018-6

Omvik, P. (1996). How smoking affects blood pressure. Blood Pressure, 5(2), 71-77.

Parrott, A. C., \& Craig, D. (1992). Cigarette smoking and nicotine gum (0, 2 and 4 mg): Effects upon four visual attention tasks. Neuropsychobiology, 25, 34-43.

Parrott, A. C., Garnham, N. J., Wesnes, K., \& Pincock, C. (1996). Cigarette smoking and abstinence: Comparative effects upon cognitive task performance and mood state over 24 hours. Human Psychopharmacology, 11, 391-400.

Pearson, J. L., Hitchman, S. C., Brose, L. S., Bauld, L., Glasser, A. M., Villanti, A. C., . . . Cohen, J. E. (2018). Recommended core items to assess e-cigarette use in populationbased surveys. Tobacco Control, 27, 341-346. doi:10.1136/tobaccocontrol-2016-053541

Perkins, K. A. (2002). Chronic tolerance to nicotine in humans and its relationship to tobacco dependence. Nicotine \& Tobacco Research, 4, 405-422. 
Perkins, K. A., Broge, M., Gerlach, D., Sanders, M., Grobe, J. E., Cherry, C., \& Wilson, A. S. (2002). Acute nicotine reinforcement, but not chronic tolerance, predicts withdrawal and relapse after quitting smoking. Health Psychology, 21(4), 332-339. doi:10.1037//0278-6133.21.4.332

Perkins, K. A., Epstein, L. H., Grobe, J., \& Fonte, C. (1994). Tobacco abstinence, smoking cues, and the reinforcing value of smoking. Pharmacology Biochemistry and Behavior, 47 107112. doi:10.1016/0091-3057(94)90118-X

Perkins, K. A., Gerlach, D., Broge, M., Grobe, J. E., Sanders, M., Fonte, C., . . Wilson, A. (2001). Dissociation of nicotine tolerance from tobacco dependence in humans. The Journal of Pharmacology and Experimental Therapeutics, 296(3), 849-856.

Perkins, K. A., Kerlitz, J. L., \& Boldry, M. C. (2017). Nicotine acutely enhances reinforcement from non-drug rewards in humans. Frontiers in Psychiatry, 8, 65. doi:10.3389/fpsyt.2017.00065

Perkins, K. A., Karelitz, J. L., \& Jao, N. C. (2013). Optimal carbon monoxide criteria to confirm 24-hr smoking abstinence. Nicotine \& Tobacco Research, 15(5), 978-982. doi:10.1093/ntr/nts205

Phillips, E., Wang, T. W., Hutsen, C. G., Corey, C. G., Apelberg, B. J., Jamal, A., . . King, B. A. (2017). Tobacco product use among adults - United States, 2015. Morbidity and Mortality Weekly Report, 66(44), 1209-1215. doi:10.15585/mmwr.mm6644a2

Piper, M. E., Baker, T. B., Benowitz, N. L., Smith, S. S., \& Jorenby, D. E. (2019). E-cigarette dependence measures in dual users: Reliability and relations with dependence criteria and e-cigarette cessation. Nicotine and Tobacco Research. Epub ahead of print. doi:10.1093/ntr/ntz040 
Pomerleau, O. F. (1995). Individual differences in sensitivity to nicotine: Implications for genetic research on nicotine dependence. Behavior Genetics, 25, 161-177.

Ramôa, C. P., Hiler, M. M., Spindle, T. R., Lopez, A. A., Karaoghlanian, N., Lipato, T., .. . Eissenberg, T. (2016). Electronic cigarette nicotine delivery can exceed that of combustible cigarettes: a preliminary report. Tobacco Control, 25, e6-e9. doi:10.1136/tobaccocontrol-2013-052447

Robinson, R. J., Hensel, E. C., Morabito, P. N., \& Roundetree, K. A. (2015). Electronic cigarette topography in the natural environment. PLoS One, 10, e0129296. doi:10.1371/journal.pone.0129296

Robinson, R. J., Hensel, E. C., Roundtree, K. A., Difrancesco, A. G., Nonnemaker, J. M., \& Lee, Y. O. (2016). Week long topography study of young adults using electronic cigarettes in their natural environment. PLoS One, 11, e0164038. doi:10.1371/journal.pone.0164038

Rubinstein, M. L., Benowitz, N. L., Auerback, G. M., \& Moscicki, A. (2009). Withdrawal in adolescent light smoker following 24-hour abstinence. Nicotine \& Tobacco Research, 11(2), 185-189. doi:10.1093/ntr/ntn028

Rupprecht, L. E., Smith, T. T., Schassburger, R. L., Buffalari, D. M., Sved, A. F., \& Donny, E. C. (2015). Behavioral mechanisms underlying nicotine reinforcement, Current Topics in Behavioral Neurosciences, 24, 19-53. doi:10.1007/978-3-319-13482-6_2

Sarter, M., Givens, B., \& Bruno, J. P. (2001). The cognitive neuroscience of sustained attention: Where top-down meets bottom-up. Brain Research Reviews, 35, 146-160.

Schneider, W., Eschman, A., \& Zuccolotto, A. (2002). E-Prime user's guide. Pittsburgh, PA: Psychology Software Tools Inc. 
Schoenborn, B. A., \& Gindi, R. M. (2015). Electronic cigarette use among adults: United States, 2014. NCHS Data Brief, 217, 1-8.

Schoenborn, B. A., \& Gindi, R. M. (2016). QuickStats: Cigarette smoking status among current adult e-cigarette users, by age group - National Health Interview Survey, United States, 2015. Morbidity and Mortality Weekly Report, 65, 1177. doi:10.15585/mmwr.m6542a7.

Schumacher, E. H., Lauber, E., Awh, E., Jonides, J., Smith, E. E., \& Koeppe, R. A. (1996). PET evidence for an amodal verbal working memory system. NeuroImage, 3(2), 79-88.

Sheehan, D. V., Lecrubier, Y., Sheehan, K. H., Amorim, P. Janavs, J., Weiller, E., Hergueta, T., . . Dunbar, G. C. (1998). The Mini-International Neuropsychiatric Interview (M.I.N.I.): The development and validation of a structured diagnostic psychiatric interview for DSM-IV and ICD-10. Clinical Psychiatry, 59, 34-57.

Shiffman, S., \& Jarvik, M. E. (1976). Smoking withdrawal symptoms in two weeks of abstinence. Psychopharmacology, 50, 35-39.

Shiffman, S., West, R., \& Gilbert, D. (2004). Recommendation for the assessment of tobacco craving and withdrawal in smoking cessation trials. Nicotine \& Tobacco Research, 6 , 599-614. doi:10.0108/14622200410001734067

Shihadeh, A., \& Eissenberg, T. (2015). Electronic cigarette effectiveness and abuse liability: predicting and regulating nicotine flux. Nicotine \& Tobacco Research, 17(2), 158-162. doi:10.1093/ntr/ntu175

Smets, J., Baeyens, F., Chaumont, M., Adriaens, K., \& Van Gucht, D. (2019). When less is more: Vaping low-nicotine vs. high-nicotine e-liquid is compensated by increased wattage and higher liquid consumption. International Journal of Environmental Research and Public Health, 16, 723. doi:10.3390/ijerph16050723 
Smith, A. (2002). Effects of caffeine on human behavior. Food and Chemical Toxicology, 40, $1243-1255$.

Smith, E. E., Jonides, J., \& Koeppe, R. A. (1996). Dissociating verbal and spatial working memory using PET. Cerebral Cortex, 6(1), 11-20.

Spindle, T. R., Hiler, M. M., Breland, A. B., Karaoghlanian, N. V., Shihadeh, A. L., \& Eissenberg, T. (2017). The influence of a mouthpiece-based topography measurement device on electronic cigarette user's plasma nicotine concentration, heart rate, and subjective effects under directed and ad libitum use conditions. Nicotine \& Tobacco Research, 19(4), 469-476. doi:10.1093/ntr/ntw174

Spindle, T. R., Talih, S., Hiler, M. M., Karaoghlanian, N., Halquist, M. S., Breland, A. B., . . Eissenberg, T. (2018). Effects of electronic cigarette liquid solvents propylene glycol and vegetable glycerin on user nicotine delivery, heart rate, subjective effects, and puff topography. Drug and Alcohol Dependence, 188, 193-199.

doi:10.1016/j.drugalcdep.2019.03.042

Srivastava, E. D., Russell, M. A., Feyerabend, C., Masterson, J. G., \& Rhodes, J. (1991). Sensitivity and tolerance to nicotine in smokers and nonsmokers. Psychopharmacology, $105,63-68$.

St. Helen, G., Ross, K. C., Dempsey, D. A., Havel, C. M., Jacob III, P. \& Benowitz, N. L. (2016). Nicotine delivery and vaping behavior during ad libitum e-cigarette access. Tobacco Regulatory Science, 2(4), 363-376. doi:10.18001/TRS.2.4.8

Stead, L. F., Perera, R., Bullen, C., Mant, D., Hartmann-Boyce, J., Cahill, K., \& Lancaster, T. (2012). Nicotine replacement therapy for smoking cessation. Cochrane Database of Systemic Reviews, 11, CD000146. doi:10.1002/14651858.CD000146.pub4 
Steinberg, L. (2007). Risk taking in adolescence: New perspectives from brain and behavioral science. Current Directions in Psychology Science, 16, 55-59. doi:10.1111/j.1467-8721.2007.00475.x

Snyder, F. R., Davis, F. C., \& Henningfield, J. E. (1989). The tobacco withdrawal syndrome: Performance decrements assessed on a computerized test battery. Drug and Alcohol Dependence, 23, 259-266.

Swan, G. E., Ward, M. M., \& Jack, L. M. (1996). Abstinence effects as predictors of 28-day relapse in smokers. Addictive Behaviors, 21(4), 481-490.

Talih, S., Balhas, Z., Eissenberg, T., Salman, R., Karaoghlanian, N., El Hallani, A., . . . Shihadeh, A. (2015). Effects of user puff topography, device voltage, and liquid nicotine concentration on electronic cigarette nicotine yield: Measurements and model predictions. Nicotine \& Tobacco Research, 17(2), 150-157. doi:10.1093/ntr/ntu174

Talih, S., Balhas, Z., Salman, R., El-Hage, R., Karaoghlanian, N. El-Hejollani, A., .. . Shihadeh, A. (2017). Transport phenomena governing nicotine emissions from electronic cigarettes: Model formulation and experimental investigation. Aerosol Science and Technology, 51, 1-11. doi:10.1080/02786826.2016.1257853

Talih, S., Salman, R., El-Hage, S., Karam, E., Karaoghlanian, N., El-Hellani, A., . . Shihadeh, A. (2019). Characteristics and toxicant emissions of JUUL electronic cigarettes. Tobacco Control. Epub ahead of print. doi:10.1136/tobaccocontrol-2018-054616

Tiffany, S. T., \& Drobes, D. J. (1991). The development and initial validation of a questionnaire on smoking urges. British Journal of Addiction, 86, 1467 - 1476.

Thornley, S., McRobbie, H., Lin, R. B., Bullen, C., Hajek, P., Laugesen, M., . . Whittaker, R. (2009). A single-blind, randomized, crossover trial of the effects of a nicotine pouch on 
the relief of tobacco withdrawal symptoms and user satisfaction. Nicotine \& Tobacco Research, 11(6), 715-721. doi:10.1093/ntr/ntp054

U. S. Food and Drug Administration (2017). Extension of certain tobacco product compliance deadlines related to the final deeming rule: Guidance for industry (revised). Rockville, MD: U. S. Food and Drug Administration.

Vansickel, A. R., Cobb, C. O., Weaver, M. F., \& Eissenberg, T. E. (2010). A clinical laboratory model for evaluating the acute effects of electronic "cigarettes": Nicotine delivery profile and cardiovascular and subjective effects. Cancer Epidemiology, Biomarkers \& Prevention, 19(8), OF1-OF9. doi:10.1158/1055-9965.EPI-10-0288.

Vansickel, A. R., \& Eissenberg, T. (2013). Electronic cigarettes: Effective nicotine delivery after acute administration. Nicotine \& Tobacco Research, 15(1), 267-270. doi:10.1093/ntr/ntr316

Vansickel, A. R., Weaver, M. F., \& Eissenberg, T. (2012). Clinical laboratory assessment of the abuse liability of an electronic cigarette. Addiction, 107, 1493-1500. doi:10.1111/j.1360-0443.2012.03791.x

Vogel, E. A., Prochaska, J. J., Ramo, D. E., Andres, J., \& Rubinstein, M. L. (2019). Adolescents' e-cigarette use: Increases in frequency, dependence, and nicotine exposure over 12 months. Journal of Adolescent Health, 64, 770-775.

doi:10.1016/j.jadohealth.2019.02.019

Wagener, T. L., Floyd, E. L., Stepanov, I., Driskill, L. M., Frank, S. G., Meier, E. Leavens, E. L., ... Queimado, L. (2017). Have combustible cigarettes met their match? The nicotine delivery profiles and harmful constituent exposures of second-generation and third- 
generation electronic cigarette users. Tobacco Control, 26, e23-e28.

doi:10.1135/tobaccocontrol-2016-053041

Wagener, T. L., Meier, E., Hale, J. J., Oliver, E. R., Warner, M. L., Driskill, L .M., . . Foster, S. (2014). Pilot investigation of changes in readiness and confidence to quit smoking after ecigarette experimentation and 1 week of use. Nicotine \& Tobacco Research, 16(1), 108114. doi:10.1093/ntr/ntt138

Ward, K. D., Garvey, A. J., \& Bliss, R. E. (1992). Evidence of transient heart rate change after smoking cessation. Psychopharmacology, 106, 337-340. doi:10.1007/bf02245414

Welsch, S. K., Smith, S. S., Wetter, D. W., Jorenby, D. E., Fiore, M. C., \& Baker, T. B. (1999). Development and validation of the Wisconsin Smoking Withdrawal Scale. Experimental and Clinical Psychopharmacology, 7(4), 354-361.

Wesnes, K., \& Warburton, D. M. (1983). Effects of smoking on rapid information processing performance. Neuropsychobiology, 9, 223-229.

Wesnes, K., \& Warburton, D. M. (1984). The effects of cigarettes of varying yield on rapid information processing performance. Psychopharmacology, 82, 338-342.

Wesnes, K., Simpson, P. M., \& Kidd, A. G. (1988). An investigation of the range of cognitive impairments induced by scopolamine 0.6mg. Human Psychopharmacology, 3, 27-43.

Wesnes, K. A., Edgar, C. J., Kezic, I., Salih, H. M., \& de Boer, P. (2013). Effects of nicotine withdrawal on cognition in a clinical trial setting. Psychopharmacology, 229, 133-140. doi:10.1007/s00213-013-3089-6

West, R., Hajek, P., Foulds, J., Nilsson, F., May, S., \& Meadows, A. (2000). A comparison of the abuse liability and dependence potential of nicotine patch, gum, spray and inhaler. Psychopharmacology, 149, 198-202. 
West, R., \& Schneider, N. (1988). Drop in heart rate following smoking cessation may be permanent. Psychopharmacology, 94, 566-568.

West, R., Ussher, M., Evans, M., \& Rashid, M. (2006). Assessing DSM-IV nicotine withdrawal symptoms: A comparison and evaluation of five different scales. Psychopharmacology, 184, 619-627. doi:10.1007/s00213-005-0216.z

Wilson, F. A., \& Wang, Y. (2017). Recent findings on the prevalence of e-cigarette use among adults in the U.S. American Journal of Preventative Medicine, 52(3), 385-390. doi:10.1016/j.amepre.2016.029

World Health Organization (1992). The ICD-10 classification of mental and behavioural disorders: Clinical descriptions and diagnostic guidelines. Geneva: World Health Organization.

Yingst, J. M., Veldeer, S., Hammett, E., Hrabovsky, S., \& Foulds, J. (2017). A method for classifying user-reported electronic cigarette liquid flavors. Nicotine \& Tobacco Research, 19, 1381-1385. doi:10.1093/ntr/ntw383

Yingst, J. M., Veldheer, S., Hrabovsky, S., Nichols, T. T., Wilson, S. J., \& Foulds, J. (2015). Factors associated with electronic cigarette users' device preferences and transition from first generation to advanced generation devices. Nicotine \& Tobacco Research, 17(10), 1242-1246. doi:10.1093/ntr/ntv052

Zack, M., Belsito, L., Scher, R., Eissenberg, T., \& Corrigall, W. A. (2001). Effects of abstinence and smoking on information processing in adolescent smokers. Psychopharmacology, 153(2), 249-257. 
Zhu, S. H., Sun, J. Y., Bonnevie, E., Cummins, S. E., Gamst, A., Yin, L., \& Lee, M. (2014). Four hundred and sixty brands of e-cigarettes and counting: Implications for product regulation. Tobacco Control, 23, iii3-iii9. doi:10.1136/tobaccocontrol-2014-051670 


\section{Table 1}

Diagnostic Criteria for Tobacco Use Disorder

A. A problematic pattern of tobacco use leading to clinically significant impairement or distress, as manifested by at least two of the following, occuring within a 12-month period

1. Tobacco is often taken in larger amounts or over a longer period than was intended.

2. There is a persitent desire or unsuccessful efforts to cut down or control tobacco use.

3. A great deal of time is spent in activities necessary to obtain or use tobacco.

4. Craving, or a strong desire or urge to use tobacco

5. Recurrent tobacco use resulting in a failure to fulfill major role obligations at work, school, or home (e.g., interference with work)

6. Continued tobacco use despite having persistent or recurrent social or interpersonal problems caused or exacerbated by the effects of tobacco (e.g., arguments with others about tobacco use)

7. Important social, occupational, or recreational activities are given up or reduced because of tobacco use.

8. Recurrent tobacco use in situations in which it is physically hazardous (e.g., smoking in bed)

9. Tobacco use is continued despite knowledge of having a persistent or recurent physical or psychological problem that is likely to have been caused by tobacco.

10. Tolerance, as defined by either of the following:

a. A need for markedly increased amounts of tobacco to achieve the desired effect.

b. A markedly diminished effect with continued use of the same amount of tobacco.

11. Withdrawal, as manifested by either of the following:

a. The characteristic withdrawal syndrome for tobacco use .

b. Tobacco (or a closely related substance, such as nicotine) is taken to relieve or avoid withdrawal symptoms. 
Table 2

Diagnostic Criteria for Tobacco Withdrawal

A. Daily use of tobacco for at least several weeks.

B. Abrupt cessation of tobacco use, or reduction in the amount of tobacco used, followed within 24 hours by four (or more) of the following signs or symptoms:

1. Irritability, frustration, or anger.

2. Anxiety.

3. Difficulty concentrating.

4. Increased appetite.

5. Restlessness.

6. Depressed mood.

7. Insomnia

C. The signs or symptoms in Criterion B cause clinically significant distress or impairement in social, occupational, or other important areas of functioning.

D. The signs or symptoms are not attributed to another medical condition and are not better explained by another mental disorder, including intoxication or withdrawal from another substance. 
Table 3

Mean (SD) or percentage for demographic, cigarette use, and ECIG use characteristics of all callers, ineligible callers, eligible callers, and completers

\begin{tabular}{|c|c|c|c|c|}
\hline & $\begin{array}{c}\text { All callers } \\
\mathrm{n}=178\end{array}$ & $\begin{array}{c}\text { Ineligible } \\
\mathrm{n}=137\end{array}$ & $\begin{array}{c}\text { Eligible } \\
\mathrm{n}=41\end{array}$ & $\begin{array}{c}\text { Completers } \\
\mathrm{n}=11\end{array}$ \\
\hline Age & $20.96(4.14)$ & $20.89(4.21)$ & $21.20(3.92)$ & $20.63(2.06)$ \\
\hline$\%$ Smokers ( $\geq 100$ cigarettes lifetime) & $27.40 \%$ & $31.62 \%$ & $12.50 \%$ & $9.10 \%$ \\
\hline Lifetime & $9633.33(30397.90)$ & $9933.33(32300.10)$ & $4660(9701.19)$ & $100.00(\mathrm{n} / \mathrm{a})$ \\
\hline Past year & $206.55(1041.00)$ & $235.92(1128.42)$ & $0.20(0.45)$ & $1.00(\mathrm{n} / \mathrm{a})$ \\
\hline Past month & $4.80(13.13)$ & $5.34(13.79)$ & $0.00(0.00)$ & $0.00(\mathrm{n} / \mathrm{a})$ \\
\hline$\%$ Nonmokers $(<100$ cigarettes lifetime $)$ & $72.60 \%$ & $68.38 \%$ & $87.50 \%$ & $90.90 \%$ \\
\hline Mean (SD) Lifetime & $14.45(20.73)$ & $14.55(20.76)$ & $13.74(20.78)$ & $8.80(10.85)$ \\
\hline Mean (SD) Past Year & $4.15(9.07)$ & $5.07(10.31)$ & $1.84(4.14)$ & $6.50(9.73)$ \\
\hline Mean (SD) Past Month & $0.45(1.34)$ & $0.62(1.53)$ & $0.00(0.00)$ & $2.33(3.46)$ \\
\hline \multicolumn{5}{|l|}{ ECIG Device Type } \\
\hline Pods (4th gen) & $76.69 \%$ & $79.67 \%$ & $67.50 \%$ & $72.72 \%$ \\
\hline Mods (3rd gen) & $22.70 \%$ & $19.51 \%$ & $32.50 \%$ & $27.27 \%$ \\
\hline Cigalike (2nd gen) & $0.61 \%$ & $0.81 \%$ & $0.00 \%$ & $0.00 \%$ \\
\hline \multicolumn{5}{|l|}{ ECIG Flavor } \\
\hline Mint/menthol & $46.47 \%$ & $47.29 \%$ & $43.90 \%$ & $45.45 \%$ \\
\hline Fruit & $25.29 \%$ & $26.36 \%$ & $21.95 \%$ & $45.45 \%$ \\
\hline Sweet/dessert & $10.59 \%$ & $10.08 \%$ & $12.20 \%$ & $9.10 \%$ \\
\hline Tobacco & $1.18 \%$ & $0.78 \%$ & $2.44 \%$ & $0.00 \%$ \\
\hline Other & $1.76 \%$ & $1.55 \%$ & $2.44 \%$ & $0.00 \%$ \\
\hline Multiple & $14.71 \%$ & $13.95 \%$ & $17.07 \%$ & $0.00 \%$ \\
\hline ECIG Nicotine Concentration (mg/ml) & $42.14(23.37)$ & $42.74(22.84)$ & $41.45(24.40)$ & $42.27(25.23)$ \\
\hline ECIG Days/Week & $6.65(1.08)$ & $6.55(1.21)$ & $6.95(0.31)$ & $7.00(0.00)$ \\
\hline ECIG Liquid Consumption (ml) & $2.14(4.21)$ & $1.93(4.18)$ & $2.79(4.30)$ & $2.58(3.82)$ \\
\hline ECIG Use Duration (months) & $16.28(13.00)$ & $15.08(11.82)$ & $20.05(15.74)$ & $21.17(9.32)$ \\
\hline ECIG \% Vegetable Glycerin & $59.62(11.07)$ & $59.76(10.79)$ & 59.19 (11.99) & $64.00(6.58)$ \\
\hline ECIG Wattage & $28.00(33.65)$ & $28.02(34.59)$ & $27.95(31.12)$ & $26.60(31.04)$ \\
\hline PSECDI Score & $8.53(4.46)$ & $7.95(4.50)$ & $10.37(3.86)$ & $11.91(3.30)$ \\
\hline
\end{tabular}


Table 4

Mean (SD) or percentage for characteristics measured at in-person screening for completers

\begin{tabular}{|c|c|}
\hline & Mean (SD) or $\%$ \\
\hline Age (years) & $20.63(2.06)$ \\
\hline$\%$ Non-Hispanic & $100.00 \%$ \\
\hline$\%$ White & $63.63 \%$ \\
\hline$\%$ Female & $63.63 \%$ \\
\hline$\%$ Student & $63.63 \%$ \\
\hline Education (years) & $14.00(1.18)$ \\
\hline Lifetime Cigarettes & $17.09(29.36)$ \\
\hline Carbon Monoxide (ppm) & $2.55(1.43)$ \\
\hline \multicolumn{2}{|l|}{ MINI Tobacco-Use Disorder } \\
\hline Severe & $63.63 \%$ \\
\hline Moderate & $18.18 \%$ \\
\hline Mild & $18.18 \%$ \\
\hline
\end{tabular}

ECIG Device Type

$\begin{array}{rr}\text { Pods (4th gen) } & 72.72 \% \\ \text { Mods (3rd gen) } & 27.27 \%\end{array}$

Nicotine Concentration $(\mathrm{mg} / \mathrm{ml})$

$42.27(25.23)$

Liquid Use / Day (ml)

$2.58(3.82)$

Duration Use (years)

$1.76(1.59)$

Flavor

$$
\begin{array}{r}
\text { Mint } \\
\text { Fruit } \\
\text { Dessert }
\end{array}
$$

Liquid Vegetable Glycerin (\%)

Wattage

PSECDI Score
$45.45 \%$

$45.45 \%$

$9.10 \%$

$64.00(6.58)$

$26.60(31.04)$

$11.91(3.30)$ 
Table 5

Descriptive statistics for variables considered for multiple linear regression to predict PSECDI scores

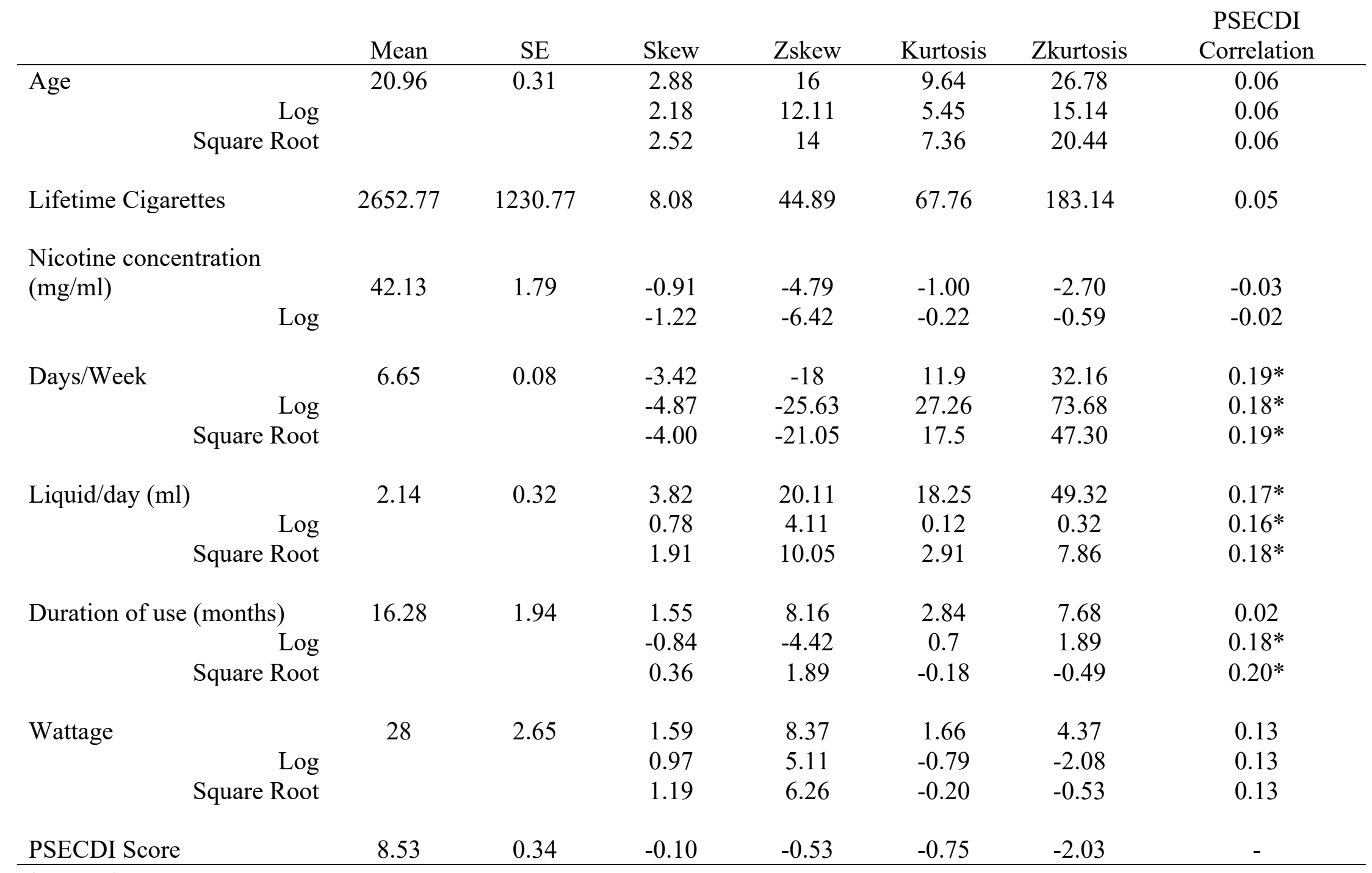


Table 6

Multiple linear regression models to predict PSECDI scores. All models significantly predicted PSECDI scores, and significant predictors are bolded

\begin{tabular}{|c|c|c|c|c|c|c|c|c|c|c|}
\hline & $F$ & $p$ & $R^{2}$ & $\begin{array}{l}\text { Adjusted } \\
\qquad R^{2}\end{array}$ & AIC & $B$ & $S E B$ & $\beta$ & $t$ & $p$ \\
\hline Model 1 & 4.39 & $<.001$ & 0.18 & 0.14 & 435.81 & & & & & \\
\hline Age (Log) & & & & & & 14.44 & 6.42 & 0.21 & 2.25 & 0.026 \\
\hline Smoke Status & & & & & & -1.15 & 0.86 & -0.12 & -1.34 & 0.18 \\
\hline Nicotine Concentration & & & & & & 0.09 & 0.30 & 0.48 & 2.84 & 0.005 \\
\hline Daily ECIG Use & & & & & & 2.81 & 1.10 & 0.21 & 2.55 & 0.012 \\
\hline Liquid Use/Day (Log) & & & & & & 1.33 & 0.99 & 0.16 & 1.34 & 0.18 \\
\hline Duration of Use (Sqrt) & & & & & & 0.53 & 0.24 & 0.18 & 2.18 & $\mathbf{0 . 0 3 1}$ \\
\hline Wattage (Sqrt) & & & & & & 3.80 & 1.53 & 0.37 & 2.48 & 0.014 \\
\hline Model 2 & 3.64 & 0.002 & 0.13 & 0.09 & 443.99 & & & & & \\
\hline Age (Log) & & & & & & 9.71 & 6.32 & 0.14 & 1.54 & 0.13 \\
\hline Smoke Status & & & & & & -0.99 & 0.88 & -0.10 & -1.13 & 0.26 \\
\hline Daily ECIG Use & & & & & & 3.59 & 1.09 & 0.27 & 3.30 & 0.001 \\
\hline Liquid Use/Day (Log) & & & & & & 0.22 & 0.93 & 0.03 & 0.24 & 0.81 \\
\hline Duration of Use (Sqrt) & & & & & & 0.53 & 0.25 & 0.18 & 2.14 & 0.03 \\
\hline Wattage (Sqrt) & & & & & & 0.75 & 1.12 & 0.07 & 0.67 & 0.50 \\
\hline Model 3 & 3.22 & 0.005 & 0.11 & 0.08 & 465.95 & & & & & \\
\hline Age (Log) & & & & & & 8.14 & 6.12 & 0.13 & 1.33 & 0.19 \\
\hline Smoke Status & & & & & & -1.07 & 0.85 & -0.11 & -1.26 & 0.21 \\
\hline Nicotine Concentration & & & & & & 0.03 & 0.02 & 0.15 & 1.18 & 0.24 \\
\hline Daily ECIG Use & & & & & & 2.8 & 1.10 & 0.21 & 2.55 & 0.012 \\
\hline
\end{tabular}


Liquid Use/Day (Log)

Duration of Use (Sqrt)

Model 4

Age (Log)

Smoke Status

Nicotine Concentration

Daily ECIG Use

Duration of Use (Sqrt)

Model 5

$$
\begin{array}{r}
\text { Age (Log) } \\
\text { Smoke Status } \\
\text { Daily ECIG Use }
\end{array}
$$

Duration of Use (Sqrt)

Model 6

$$
\begin{array}{r}
\text { Age (Log) } \\
\text { Daily ECIG Use }
\end{array}
$$

Duration of Use (Sqrt)

\section{Model 7}

$$
\text { Age (Log) }
$$

Nicotine Concentration Daily ECIG Use

Liquid Use/Day (Log)

Duration of Use (Sqrt) Wattage (Sqrt)

$\begin{array}{llllc}1.48 & 0.99 & 0.18 & 1.50 & 0.14 \\ \mathbf{0 . 4 8} & \mathbf{0 . 2 4} & \mathbf{0 . 1 6} & \mathbf{2 . 0 3} & \mathbf{0 . 0 4 4}\end{array}$

$\begin{array}{lllll}3.40 & 0.006 & 0.10 & 0.07 & 472.72\end{array}$

$\begin{array}{lllll}8.04 & 5.91 & 0.13 & 1.36 & 0.18\end{array}$

$\begin{array}{lllll}-0.79 & 0.84 & -0.08 & -0.08 & 0.35\end{array}$

$\begin{array}{lllll}0.08 & 0.02 & 0.04 & 0.04 & 0.66\end{array}$

$\begin{array}{lllll}3.27 & 1.04 & 0.24 & 0.24 & 0.002\end{array}$

$\begin{array}{lllll}0.53 & 0.23 & 0.18 & 0.18 & 0.02\end{array}$

$\begin{array}{lllll}4.30 & 0.002 & 0.10 & 0.08 & 474.99\end{array}$

$\begin{array}{ccccc}6.83 & 5.24 & 0.11 & 1.30 & 0.19 \\ -0.80 & 0.83 & -0.08 & -0.97 & 0.34 \\ \mathbf{3 . 2 8} & \mathbf{1 . 0 3} & \mathbf{0 . 2 4} & \mathbf{3 . 2 0} & \mathbf{0 . 0 0 2} \\ \mathbf{0 . 5 3} & \mathbf{0 . 2 2} & \mathbf{0 . 1 8} & \mathbf{2 . 3 4} & \mathbf{0 . 0 2}\end{array}$

$\begin{array}{lllll}5.02 & 0.002 & 0.09 & 0.07 & 491.58\end{array}$

$\begin{array}{llllc}1.89 & 4.71 & 0.03 & 0.40 & 0.69 \\ \mathbf{3 . 1 8} & \mathbf{1 . 0 5} & \mathbf{0 . 2 3} & \mathbf{3 . 0 3} & \mathbf{0 . 0 0 3} \\ \mathbf{0 . 5 2} & \mathbf{0 . 2 3} & \mathbf{0 . 1 8} & \mathbf{2 . 3 2} & \mathbf{0 . 0 2}\end{array}$

$\begin{array}{lllll}4.27 & 0.001 & 0.15 & 0.11 & 454.83\end{array}$

$\begin{array}{lllll}7.24 & 6.01 & 0.10 & 1.20 & 0.23\end{array}$

$\begin{array}{lllll}0.09 & 0.03 & 0.44 & 2.59 & 0.01\end{array}$

$\begin{array}{lllll}2.78 & 1.14 & 0.20 & 2.44 & 0.02\end{array}$

$\begin{array}{lllll}0.97 & 1.00 & 0.12 & 0.97 & 0.33\end{array}$

$\begin{array}{lllll}0.48 & 0.25 & 0.16 & 1.98 & 0.05\end{array}$

\begin{tabular}{lllll}
4.11 & 1.58 & 0.39 & 2.60 & 0.01 \\
\hline
\end{tabular} 
Table 7

Repeated-measures ANOVA results for heart rate and subjective outcomes. Significant main effects and interactions are bolded.

Condition $^{\mathrm{a}}$

Time $^{b}$

Condition $\mathrm{x}$ Time ${ }^{\mathrm{b}}$

\begin{tabular}{lccccccccc} 
Outcome Measure & $F$ & $p$ & $\eta_{\mathrm{p}}{ }^{2}$ & $F$ & $p$ & $\eta_{\mathrm{p}}{ }^{2}$ & $F$ & $p$ & $\eta_{\mathrm{p}}{ }^{2}$ \\
\hline Heart Rate & $\mathbf{9 . 8 2}$ & $\mathbf{0 . 0 1 1}$ & $\mathbf{0 . 5 0}$ & $\mathbf{2 4 . 3 0}$ & $<\mathbf{0 . 0 0 1}$ & $\mathbf{0 . 7 1}$ & 1.14 & 0.35 & 0.10
\end{tabular}

MNWS

\begin{tabular}{|c|c|c|c|c|c|c|c|c|c|}
\hline Anxious & 1.10 & 0.32 & 0.10 & 1.34 & 0.26 & 0.12 & 0.23 & 0.88 & 0.02 \\
\hline Craving & 4.24 & 0.07 & 0.30 & 10.36 & $<0.001$ & 0.51 & 4.04 & 0.014 & 0.29 \\
\hline Depressed & 0.10 & 0.76 & 0.01 & 1.54 & 0.23 & 0.13 & 0.38 & 0.70 & 0.04 \\
\hline Difficulty Concentrating & 0.53 & 0.48 & 0.05 & 4.79 & 0.002 & 0.32 & 1.57 & 0.20 & 0.14 \\
\hline Drowsy & 0.83 & 0.38 & 0.08 & 0.74 & 0.49 & 0.07 & 0.74 & 0.58 & 0.07 \\
\hline Hunger & 0.01 & 0.94 & 0.00 & 0.81 & 0.49 & 0.08 & 1.19 & 0.33 & 0.11 \\
\hline Impatient & 1.01 & 0.34 & 0.09 & 6.05 & 0.006 & 0.38 & 1.43 & 0.26 & 0.13 \\
\hline Irritable & 0.93 & 0.36 & 0.09 & 3.21 & 0.01 & 0.24 & 0.27 & 0.79 & 0.03 \\
\hline Restless & 4.19 & 0.07 & 0.30 & 2.28 & 0.08 & 0.19 & 1.13 & 0.36 & 0.10 \\
\hline Desire Sweets & 0.12 & 0.73 & 0.01 & 2.04 & 0.14 & 0.17 & 0.53 & 0.64 & 0.05 \\
\hline Urges & 4.83 & 0.053 & 0.33 & 7.14 & $<0.001$ & 0.42 & 2.53 & 0.042 & 0.20 \\
\hline \multicolumn{10}{|l|}{ U-Brief } \\
\hline Factor 1 & 2.59 & 0.14 & 0.21 & 8.28 & 0.002 & 0.45 & 1.96 & 0.056 & 0.22 \\
\hline Factor 2 & 7.46 & 0.02 & 0.43 & 3.39 & 0.02 & 0.25 & 2.22 & 0.091 & 0.18 \\
\hline Confused & 1.09 & 0.32 & 0.10 & 2.43 & 0.08 & 0.20 & 0.22 & 0.83 & 0.02 \\
\hline Dizzy & 2.41 & 0.15 & 0.19 & 5.33 & 0.018 & 0.35 & 1.05 & 0.39 & 0.10 \\
\hline
\end{tabular}




\begin{tabular}{rccccccccc} 
Headache & 4.56 & 0.06 & 0.31 & 2.10 & 0.17 & 0.17 & 1.15 & 0.35 & 0.10 \\
Heart Pound & 0.30 & 0.60 & 0.03 & $\mathbf{4 . 4 5}$ & $\mathbf{0 . 0 2}$ & $\mathbf{0 . 3 1}$ & 1.41 & 0.26 & 0.12 \\
Lightheaded & 4.90 & 0.051 & 0.33 & $\mathbf{5 . 9 8}$ & $\mathbf{0 . 0 2}$ & $\mathbf{0 . 3 7}$ & 0.67 & 0.60 & 0.06 \\
Nausea & 3.51 & 0.09 & 0.26 & 3.36 & 0.07 & 0.25 & 0.22 & 0.85 & 0.02 \\
Nervous & 0.58 & 0.46 & 0.06 & 2.31 & 0.09 & 0.19 & 1.36 & 0.28 & 0.12 \\
Salivate & 0.01 & 0.93 & 0.00 & 2.37 & 0.11 & 0.19 & 0.31 & 0.81 & 0.03 \\
Sweaty & 0.11 & 0.74 & 0.01 & 0.63 & 0.52 & 0.06 & 1.13 & 0.35 & 0.10 \\
Weak & 0.81 & 0.39 & 0.08 & 2.97 & 0.11 & 0.23 & $\mathbf{3 . 2 2}$ & $\mathbf{0 . 0 4 2}$ & $\mathbf{0 . 2 4}$ \\
& & & & & & & & & \\
Awake & 1.02 & 0.34 & 0.09 & 0.77 & 0.50 & 0.07 & 1.29 & 0.28 & 0.11 \\
Calm & 3.98 & 0.07 & 0.29 & $\mathbf{3 . 8 5}$ & $\mathbf{0 . 0 0 3}$ & $\mathbf{0 . 2 8}$ & 0.52 & 0.69 & 0.05 \\
Concentrate & 1.80 & 0.21 & 0.15 & 1.03 & 0.41 & 0.09 & 0.61 & 0.67 & 0.06 \\
Dizzy & 0.14 & 0.71 & 0.01 & 1.76 & 0.18 & 0.15 & 0.83 & 0.50 & 0.08 \\
Pleasant & 3.50 & 0.09 & 0.26 & 3.29 & 0.08 & 0.25 & $\mathbf{3 . 6 1}$ & $\mathbf{0 . 0 3 4}$ & $\mathbf{0 . 2 7}$ \\
Satisfy & 3.91 & 0.08 & 0.28 & 2.16 & 0.14 & 0.18 & $\mathbf{3 . 7 2}$ & $\mathbf{0 . 0 1 6}$ & $\mathbf{0 . 2 7}$ \\
Sick & 0.82 & 0.39 & 0.08 & 1.05 & 0.39 & 0.10 & 0.38 & 0.66 & 0.04 \\
Taste Good & 3.64 & 0.09 & 0.27 & 2.65 & 0.11 & 0.21 & 2.07 & 0.16 & 0.17 \\
\hline
\end{tabular}

${ }^{\mathrm{a}} d f=1,10 ;{ }^{\mathrm{b}}$ Heart Rate $d f=7,70 ;$ Subjectives $\underline{d f}=6,60$ 
Table 8

Mean (SD) for best cognitive performance during training.

Mean (SD)

\begin{tabular}{|c|c|}
\hline \multicolumn{2}{|l|}{ RVIP } \\
\hline$\%$ Correct & $54.72(16.58)$ \\
\hline Reaction Time & $510.65(95.93)$ \\
\hline Errors Commission & $8.09(10.15)$ \\
\hline Errors Omission & $21.73(8.01)$ \\
\hline \multicolumn{2}{|l|}{ N-back } \\
\hline 2-back Accuracy & $97.82(4.49)$ \\
\hline 3-back Accuracy & $67.14(14.30)$ \\
\hline 2-back Reaction Time & $484.49(104.87)$ \\
\hline 3-back Reaction Time & $600.77(112.58)$ \\
\hline \multicolumn{2}{|l|}{ Cued Go/No-Go } \\
\hline No-Go Congruent Accuracy & $98.55(3.36)$ \\
\hline No-Go Incongruent Accuracy & $98.73(1.90)$ \\
\hline Go Reaction Time & $328.69(27.51)$ \\
\hline
\end{tabular}


Table 9

Paired-samples t-test results comparing 120 minutes ECIG abstinence to baseline ECIG abstinence and 120 minutes ad lib ECIG use

120 min abstinence - Baseline abstinence

\begin{tabular}{|c|c|c|c|c|c|c|}
\hline & $t$ & $p$ & $d$ & $t$ & $p$ & $d$ \\
\hline \multicolumn{7}{|l|}{ RVIP } \\
\hline$\%$ Correct & -1.16 & 0.27 & 0.20 & 0.62 & 0.55 & 0.14 \\
\hline Reaction Time & 0.94 & 0.37 & 0.15 & -0.53 & 0.61 & 0.15 \\
\hline Errors Commission & 0.52 & 0.62 & 0.10 & -0.89 & 0.49 & 0.22 \\
\hline Errors Omission & 1.17 & 0.27 & 0.20 & -0.63 & 0.54 & 0.14 \\
\hline \multicolumn{7}{|l|}{ N-back } \\
\hline 2-back Accuracy & -1.08 & 0.30 & 0.30 & 0.23 & 0.82 & 0.07 \\
\hline 3-back Accuracy & 3.64 & 0.005 & 0.87 & 2.04 & 0.07 & 0.44 \\
\hline 2-back Reaction Time & -1.61 & 0.14 & 0.26 & 0.45 & 0.66 & 0.11 \\
\hline 3-back Reaction Time & 0.81 & 0.94 & 0.02 & 0.34 & 0.74 & 0.06 \\
\hline \multicolumn{7}{|l|}{ Cued Go/No-Go } \\
\hline No-Go Congruent Accuracy & 0.80 & 0.44 & 0.36 & 0.00 & 1.00 & 0.00 \\
\hline No-Go Incongruent Accuracy & -3.99 & 0.003 & 1.08 & -2.68 & 0.023 & 0.90 \\
\hline Go Reaction Time & 0.73 & 0.48 & 0.13 & -0.64 & 0.54 & 0.014 \\
\hline
\end{tabular}

120 minutes abstinence - 120 minutes ad lib

$d f=10$

.


Table 10

Mean (SE) cognitive performance for the ad lib and abstinent conditions at baseline and 120 minutes

\begin{tabular}{|c|c|c|c|c|}
\hline & \multicolumn{2}{|c|}{ Ad lib } & \multicolumn{2}{|c|}{ Abstinent } \\
\hline & Baseline & 120 minutes & Baseline & 120 minutes \\
\hline \multicolumn{5}{|l|}{ RVIP } \\
\hline$\%$ Correct & $50.55(4.10)$ & $49.45(4.57)$ & $55.27(5.24)$ & $51.72(5.49)$ \\
\hline Reaction Time & $433.03(12.72)$ & $464.57(22.25)$ & $463.49(16.53)$ & $454.37(20.07)$ \\
\hline Errors Commission & $3.27(1.38)$ & $4.18(1.62)$ & $5.00(2.16)$ & $5.81(2.71)$ \\
\hline Errors Omission & $23.72(1.96)$ & $24.27(2.17)$ & $21.45(2.51)$ & $23.18(2.63)$ \\
\hline \multicolumn{5}{|l|}{ N-back } \\
\hline 2-back Accuracy & $93.45(2.19)$ & $89.27(3.40)$ & $93.27(3.07)$ & $90.09(3.33)$ \\
\hline 3-back Accuracy & $54.63(4.46)$ & $57.90(8.53)$ & $51.09(5.95)$ & $69.00(6.49)$ \\
\hline 2-back Reaction Time & $483.40(33.29)$ & $506.61(32.76)$ & $487.93(35.29)$ & $519.47(35.82)$ \\
\hline 3-back Reaction Time & $540.03(52.78)$ & $591.45(51.97)$ & $598.20(32.22)$ & $599.79(31.78)$ \\
\hline \multicolumn{5}{|l|}{ Cued Go/No-Go } \\
\hline No-Go Congruent Accuracy & $98.55(0.81)$ & $99.64(0.36)$ & $98.91(0.78)$ & $99.64(0.36)$ \\
\hline No-Go Incongruent Accuracy & $98.82(0.42)$ & $98.73(0.41)$ & $99.00(0.40)$ & $97.36(0.51)$ \\
\hline Go Reaction Time & $337.21(9.71)$ & $339.02(10.89)$ & $328.66(8.97)$ & $333.53(13.41)$ \\
\hline
\end{tabular}


Table 11

Mean (SD) change in cognitive performance from baseline to 120 minutes for the ad lib and abstinent conditions

\begin{tabular}{|c|c|c|}
\hline & Ad lib & Abstinent \\
\hline \multicolumn{3}{|l|}{ RVIP } \\
\hline$\%$ Correct & $-1.09(10.90)$ & $-3.55(10.16)$ \\
\hline Reaction Time & $31.54(64.36)$ & $-9.11(32.17)$ \\
\hline Errors Commission & $.91(1.64)$ & $.82(5.27)$ \\
\hline Errors Omission & $.55(5.13)$ & $1.73(4.90)$ \\
\hline \multicolumn{3}{|l|}{ N-back } \\
\hline 2-back Accuracy & $-4.18(12.84)$ & $-3.18(9.73)$ \\
\hline 3-back Accuracy & $3.27(19.66)$ & $17.91(16.31)$ \\
\hline 2-back Reaction Time & $23.21(79.61)$ & $31.54(65.13)$ \\
\hline 3-back Reaction Time & $51.41(106.64)$ & $1.59(64.72)$ \\
\hline \multicolumn{3}{|l|}{ Cued Go/No-Go } \\
\hline No-Go Congruent Accuracy & $1.09(3.14)$ & $0.73(3.00)$ \\
\hline No-Go Incongruent Accuracy & $-.09(1.87)$ & $-1.64(1.36)$ \\
\hline Go Reaction Time & $1.81(16.13)$ & $4.87(22.20)$ \\
\hline
\end{tabular}


Table 12

Mean (SD) cognitive performance for session 1 and session 2 at baseline and 120 minutes

Session 1

\begin{tabular}{|c|c|c|c|c|}
\hline & Baseline & 120 minutes & Baseline & 120 minutes \\
\hline \multicolumn{5}{|l|}{ RVIP } \\
\hline$\%$ Correct & $53.27(16.79)$ & $52.91(14.77)$ & $52.55(14.70)$ & $48.27(18.29)$ \\
\hline Reaction Time & $441.01(53.34)$ & $464.37(73.40)$ & $455.51(48.33)$ & $454.57(67.04)$ \\
\hline Errors Commission & $4.72(7.32)$ & $5.91(8.88)$ & $3.55(4.44)$ & $4.09(5.54)$ \\
\hline Errors Omission & $22.45(8.04)$ & $22.63(7.05)$ & $22.73(7.06)$ & $24.82(8.75)$ \\
\hline \multicolumn{5}{|l|}{ N-back } \\
\hline 2-back Accuracy & $95.27(7.72)$ & $92.73(10.30)$ & $91.45(9.44)$ & $86.64(11.11)$ \\
\hline 3-back Accuracy & $54.27(17.62)$ & $63.91(25.12)$ & $51.45(17.33)$ & $63.00(26.47)$ \\
\hline 2-back Reaction Time & $490.81(112.35)$ & $492.86(93.74)$ & $480.52(114.99)$ & $533.23(127.76)$ \\
\hline 3-back Reaction Time & $587.01(146.09)$ & $605.72(151.85)$ & $551.23(147.92)$ & $585.52(132.59)$ \\
\hline \multicolumn{5}{|l|}{ Cued Go/No-Go } \\
\hline No-Go Congruent Accuracy & $98.55(2.70)$ & $99.64(1.21)$ & $98.91(2.59)$ & $99.64(1.21)$ \\
\hline No-Go Incongruent Accuracy & $99.36(0.92)$ & $98.55(1.57)$ & $98.45(1.57)$ & $97.55(1.63)$ \\
\hline Go Reaction Time & $329.74(26.95)$ & $333.60(44.37)$ & $336.13(34.81)$ & $338.95(36.23)$ \\
\hline
\end{tabular}

Session 2

.

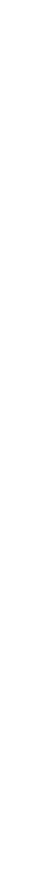


Table 13

Mean (SE) MCP crossover point for the ad lib and abstinence conditions at baseline, 75 minutes, and 150 minutes

\begin{tabular}{cccc} 
& Baseline & $75 \mathrm{~min}$ & $150 \mathrm{~min}$ \\
\hline Ad lib & $1.73(0.57)$ & $1.47(0.44)$ & $1.76(0.54)$
\end{tabular}

\begin{tabular}{llll} 
Abstinent & $1.51(0.41)$ & $1.72(0.43)$ & $2.15(0.50)$ \\
\hline
\end{tabular}


Table 14

Puff topography clustering patterns for each participant during the ad lib condition

\begin{tabular}{|c|c|c|c|c|c|c|c|c|c|c|c|}
\hline \multirow[b]{2}{*}{ Participant } & \multicolumn{4}{|c|}{ Frequency of puff clusters } & \multicolumn{3}{|c|}{ Average number of puffs per cluster } & \multicolumn{4}{|c|}{ Percent of total puffs in session (\%) } \\
\hline & 1 puff & $2-5$ puffs & $6-10$ puffs & $>10$ puffs & $2-5$ puffs & $6-10$ puffs & $>10$ puffs & 1 puff & $2-5$ puffs & $6-10$ puffs & $>10$ puffs \\
\hline 501 & 4 & 13 & 2 & 0 & 3.85 & 6.00 & $\mathrm{n} / \mathrm{a}$ & 6.15 & 76.92 & 18.46 & 0.00 \\
\hline 502 & 24 & 21 & 0 & 0 & 2.43 & $\mathrm{n} / \mathrm{a}$ & $\mathrm{n} / \mathrm{a}$ & 32.00 & 68.00 & 0.00 & 0.00 \\
\hline 504 & 19 & 16 & 0 & 0 & 2.44 & $\mathrm{n} / \mathrm{a}$ & $\mathrm{n} / \mathrm{a}$ & 32.76 & 67.24 & 0.00 & 0.00 \\
\hline 505 & 0 & 13 & 3 & 0 & 3.62 & 7.67 & $\mathrm{n} / \mathrm{a}$ & 0.00 & 61.04 & 29.87 & 0.00 \\
\hline 507 & 17 & 1 & 0 & 0 & 2.00 & $\mathrm{n} / \mathrm{a}$ & $\mathrm{n} / \mathrm{a}$ & 88.24 & 11.76 & 0.00 & 0.00 \\
\hline 510 & 22 & 5 & 0 & 0 & 2.60 & $\mathrm{n} / \mathrm{a}$ & $\mathrm{n} / \mathrm{a}$ & 62.86 & 37.14 & 0.00 & 0.00 \\
\hline 513 & 28 & 9 & 0 & 0 & 2.44 & $\mathrm{n} / \mathrm{a}$ & $\mathrm{n} / \mathrm{a}$ & 56.00 & 44.00 & 0.00 & 0.00 \\
\hline 514 & 27 & 12 & 0 & 0 & 2.67 & $\mathrm{n} / \mathrm{a}$ & $\mathrm{n} / \mathrm{a}$ & 45.76 & 54.24 & 0.00 & 0.00 \\
\hline 515 & 15 & 3 & 1 & 0 & 2.33 & 6.00 & $\mathrm{n} / \mathrm{a}$ & 53.57 & 24.00 & 21.43 & 0.00 \\
\hline Mean & 17.33 & 10.33 & 0.67 & 0.00 & 2.71 & 6.56 & $\mathrm{n} / \mathrm{a}$ & 41.93 & 49.37 & 7.75 & 0.00 \\
\hline SD & 9.75 & 6.46 & 1.12 & 0.00 & 0.61 & 0.96 & $\mathrm{n} / \mathrm{a}$ & 27.73 & 21.86 & 12.00 & 0.00 \\
\hline
\end{tabular}


Table 15

Multiple linear regressions to predict withdrawal based on puff topography. Significant models and predictors are blooded.

\begin{tabular}{|c|c|c|c|c|c|c|c|c|c|c|}
\hline & & $R$ & $R^{2}$ & $F$ & $p$ & $B$ & $S E B$ & $\beta$ & $t$ & $p$ \\
\hline Heart Rate & & 0.42 & 0.18 & 2.93 & 0.03 & & & & & \\
\hline & Puff Number & & & & & 0.74 & 0.44 & 0.43 & 1.69 & 0.10 \\
\hline & Puff Duration & & & & & -2.76 & 1.05 & -0.33 & -2.52 & 0.011 \\
\hline & IPI & & & & & -0.003 & 0.003 & -0.13 & -0.89 & 0.38 \\
\hline & Cluster & & & & & -3.35 & 1.48 & -0.54 & -2.27 & 0.03 \\
\hline \multicolumn{11}{|l|}{ MNWS } \\
\hline Anxious & & 0.32 & 0.11 & 1.59 & 0.19 & & & & & \\
\hline & Puff Number & & & & & -0.62 & 0.53 & -0.31 & -1.17 & 0.25 \\
\hline & Puff Duration & & & & & 2.60 & 1.27 & 0.27 & 2.05 & 0.046 \\
\hline & IPI & & & & & -0.004 & 0.003 & -0.19 & -1.23 & 0.224 \\
\hline & Cluster & & & & & 2.06 & 1.78 & 0.29 & 1.16 & 0.25 \\
\hline \multirow[t]{5}{*}{ Craving } & & 0.48 & 0.23 & 3.92 & 0.007 & & & & & \\
\hline & Puff Number & & & & & 1.65 & 1.15 & 0.36 & 1.44 & 0.16 \\
\hline & Puff Duration & & & & & 8.03 & 2.75 & 0.36 & 2.92 & 0.005 \\
\hline & IPI & & & & & 0.02 & 0.008 & 0.36 & 2.46 & 0.017 \\
\hline & Cluster & & & & & 0.07 & 3.85 & 0.004 & 0.02 & 0.99 \\
\hline \multirow[t]{5}{*}{ Depressed } & & 0.16 & 0.03 & 0.34 & 0.85 & & & & & \\
\hline & Puff Number & & & & & 0.08 & 0.32 & 0.07 & 0.02 & 0.98 \\
\hline & Puff Duration & & & & & 0.22 & 0.77 & 0.04 & 0.29 & 0.78 \\
\hline & IPI & & & & & -0.002 & 0.002 & -0.16 & -0.96 & 0.34 \\
\hline & Cluster & & & & & -0.47 & 1.07 & -0.11 & -0.44 & 0.66 \\
\hline
\end{tabular}


Difficulty Concentrate
Puff Number
Puff Duration
IPI
Cluster

Drowsy

Puff Number

Puff Duration

IPI

Cluster

\section{Hunger}

Puff Number

Puff Duration

IPI

Cluster

Impatient

Puff Number

Puff Duration

IPI

Cluster

Irritable

Puff Number

Puff Duration

IPI

Cluster $\begin{array}{llll}0.29 & 0.08 & 1.24 & 0.31\end{array}$

$\begin{array}{ccccc}-1.06 & 0.92 & -0.31 & -1.16 & 0.25 \\ 4.12 & 2.2 & 0.25 & 1.88 & 0.07 \\ -0.005 & 0.006 & -0.14 & -0.91 & 0.37 \\ 3.39 & 3.08 & 0.28 & 1.1 & 0.28\end{array}$

0.58

0.33

$6.71<.001$

$\begin{array}{ccccc}-1.27 & 1.12 & -0.26 & -1.13 & 0.26 \\ \mathbf{1 1 . 7 5} & \mathbf{2 . 6 9} & \mathbf{0 . 4 9} & \mathbf{4 . 3 7} & <.001 \\ \mathbf{- 0 . 0 2} & \mathbf{0 . 0 0 7} & \mathbf{- 0 . 3 2} & \mathbf{- 2 . 3 9} & \mathbf{0 . 0 2 1} \\ 0.31 & 3.77 & 0.017 & 0.081 & 0.94\end{array}$

$\begin{array}{llll}0.54 & 0.29 & 5.53 & 0.001\end{array}$

$\begin{array}{ccccc}-1.15 & 1.25 & -0.22 & -0.92 & 0.36 \\ \mathbf{1 1 . 4 2} & \mathbf{3 . 0 1} & \mathbf{0 . 4 4} & \mathbf{3 . 8 0} & <.001 \\ -0.01 & 0.008 & -0.17 & -1.24 & 0.22 \\ \mathbf{8 . 6 0} & \mathbf{4 . 2 1} & \mathbf{0 . 4 5} & \mathbf{2 . 0 4} & \mathbf{0 . 0 4 6}\end{array}$

$\begin{array}{llll}0.65 & 0.42 & 9.93<.001\end{array}$

$\begin{array}{ccccc}0.35 & 0.70 & 0.11 & 0.50 & 0.62 \\ \mathbf{1 0 . 0 8} & \mathbf{1 . 6 7} & \mathbf{0 . 6 4} & \mathbf{6 . 0 5} & <.001 \\ 0.003 & 0.005 & 0.08 & 0.62 & 0.54 \\ 2.04 & 2.34 & 0.17 & 0.87 & 0.39\end{array}$

$\begin{array}{llll}0.40 & 0.16 & 2.54 & 0.05\end{array}$

$\begin{array}{ccccc}\mathbf{- 0 . 7 5} & \mathbf{0 . 3 7} & \mathbf{- 0 . 5 3} & \mathbf{- 2 . 0 5} & \mathbf{0 . 0 4 6} \\ \mathbf{2 . 1 0} & \mathbf{0 . 8 8} & \mathbf{0 . 3 0} & \mathbf{2 . 3 9} & \mathbf{0 . 0 2 1} \\ -0.003 & 0.002 & -0.18 & -1.21 & 0.23 \\ \mathbf{2 . 6 9} & \mathbf{1 . 2 3} & \mathbf{0 . 5 2} & \mathbf{2 . 1 8} & \mathbf{0 . 0 3 3}\end{array}$




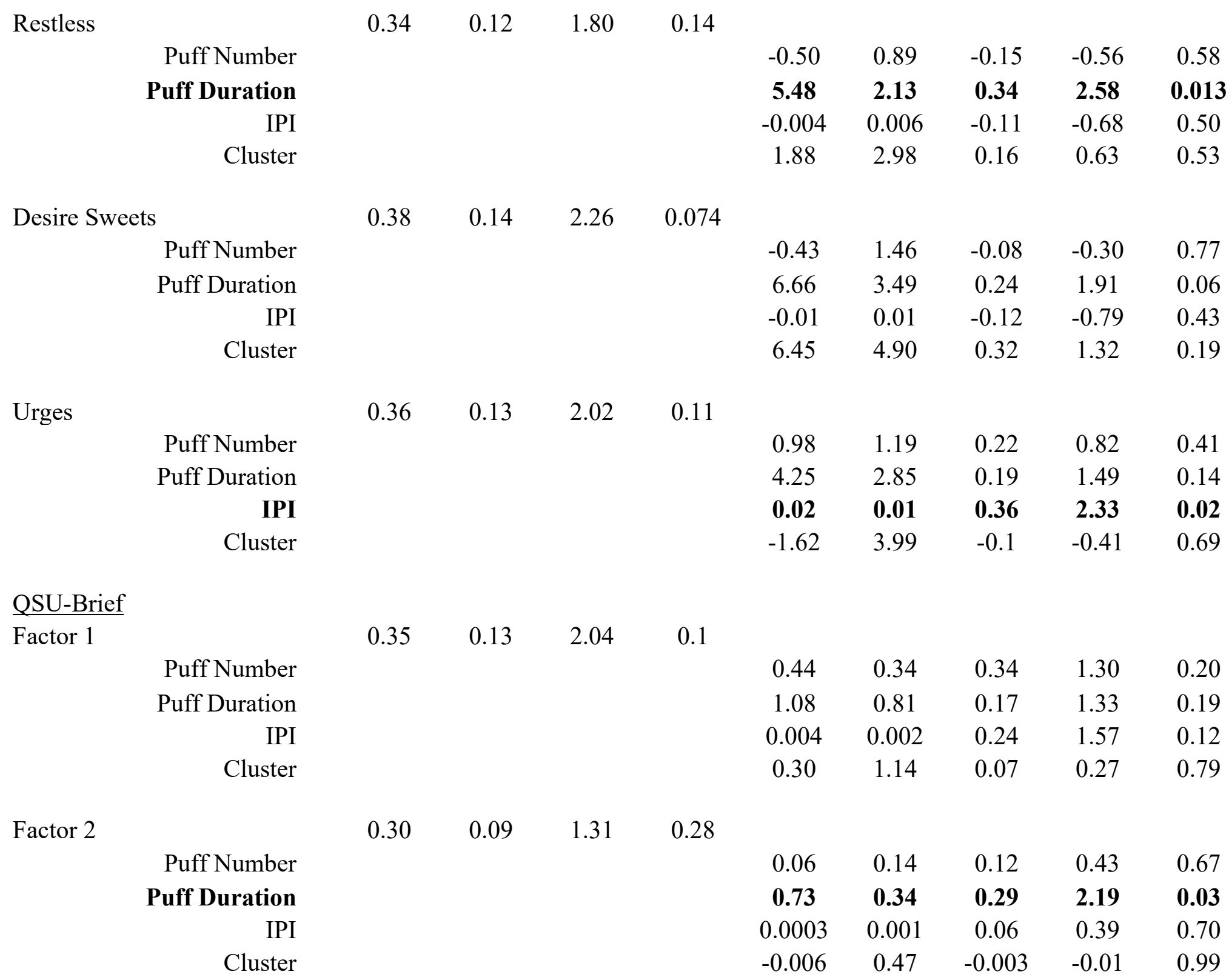




\section{$\underline{\text { DENS }}$}

Confused

Puff Number

Puff Duration

IPI

Cluster

Dizzy

Puff Number

Puff Duration

IPI

Cluster

Headache

Puff Number

Puff Duration

IPI

Cluster

Heart Pound

Puff Number

Puff Duration

IPI

Cluster

Lightheaded

Puff Number

Puff Duration

$\begin{array}{llll}0.33 & 0.11 & 1.60 & 0.19\end{array}$

$\begin{array}{ccccc}-0.40 & 0.25 & -0.43 & -1.60 & 0.12 \\ -0.26 & 0.59 & -0.06 & -0.44 & 0.66 \\ -0.002 & 0.002 & -0.18 & -1.13 & 0.26 \\ \mathbf{1 . 7 2} & \mathbf{0 . 8 3} & \mathbf{0 . 5 1} & \mathbf{2 . 0 7} & \mathbf{0 . 0 4}\end{array}$

$\begin{array}{llll}0.26 & 0.07 & 0.96 & 0.44\end{array}$

$\begin{array}{ccccc}-1.95 & 1.31 & -0.41 & -1.49 & 0.14 \\ 1.09 & 3.14 & 0.05 & 0.35 & 0.73 \\ -0.01 & 0.009 & -0.23 & -1.44 & 0.16 \\ 6.35 & 4.40 & 0.37 & 1.44 & 0.16\end{array}$

$\begin{array}{llll}0.30 & 0.09 & 1.35 & 0.27\end{array}$

$\begin{array}{ccccc}-0.78 & 0.78 & -0.27 & -1.01 & 0.32 \\ 2.57 & 1.86 & 0.18 & 1.39 & 0.17 \\ -0.008 & 0.005 & -0.25 & -1.61 & 0.11 \\ 0.39 & 2.61 & 0.04 & 0.15 & 0.88\end{array}$

$\begin{array}{llll}0.21 & 0.04 & 0.61 & 0.66\end{array}$

$\begin{array}{ccccc}-0.20 & 0.54 & -0.10 & -0.38 & 0.71 \\ 0.64 & 1.30 & 0.07 & 0.50 & 0.62 \\ -0.005 & 0.004 & -0.23 & -1.43 & 0.16 \\ 0.12 & 1.83 & 0.02 & 0.07 & 0.95\end{array}$

$\begin{array}{llll}0.28 & 0.08 & 1.15 & 0.34\end{array}$

$\begin{array}{ccccc}-1.64 & 1.19 & -0.37 & -1.37 & 0.18 \\ 0.33 & 2.86 & 0.02 & 0.12 & 0.91\end{array}$




\author{
IPI \\ Cluster
}

Nausea

Puff Number

Puff Duration

IPI

Cluster

Nervous

Puff Number

Puff Duration

IPI

Cluster

Salivate

Puff Number

Puff Duration

IPI

Cluster

Sweaty

$$
\begin{array}{r}
\text { Puff Number } \\
\text { Puff Duration } \\
\text { IPI } \\
\text { Cluster }
\end{array}
$$

Weak

Puff Number

Puff Duration

$\begin{array}{ccccc}-0.01 & 0.008 & -0.23 & -1.47 & 0.15 \\ 6.25 & 4.01 & 0.39 & 1.56 & 0.13\end{array}$

$\begin{array}{llll}0.23 & 0.05 & 0.74 & 0.57\end{array}$

$\begin{array}{ccccc}-1.18 & 0.97 & -0.34 & -1.21 & 0.23 \\ 0.63 & 2.33 & 0.04 & 0.27 & 0.79 \\ -0.008 & 0.006 & -0.20 & -1.26 & 0.21 \\ 4.03 & 3.27 & 0.31 & 1.23 & 0.22\end{array}$

$\begin{array}{llll}0.44 & 0.19 & 3.16 & 0.02\end{array}$

$\begin{array}{ccccc}\mathbf{- 1 . 0 3} & \mathbf{0 . 3 5} & \mathbf{- 0 . 7 6} & \mathbf{- 2 . 9 8} & \mathbf{0 . 0 0 4} \\ 0.56 & 0.83 & 0.08 & 0.67 & 0.50 \\ -0.004 & 0.002 & -0.27 & -1.83 & 0.07 \\ \mathbf{3 . 7 2} & \mathbf{1 . 1 6} & \mathbf{0 . 7 5} & \mathbf{3 . 2} & \mathbf{0 . 0 0 2}\end{array}$

$\begin{array}{llll}0.42 & 0.18 & 2.87 & 0.03\end{array}$

$\begin{array}{ccccc}-0.37 & 0.37 & -0.26 & -1.00 & 0.32 \\ -0.09 & 0.89 & -0.01 & -0.10 & 0.92 \\ -0.004 & 0.002 & -0.25 & -1.64 & 0.11 \\ \mathbf{2 . 5 0} & \mathbf{1 . 2 5} & \mathbf{0 . 4 8} & \mathbf{2 . 0 0} & \mathbf{0 . 0 5}\end{array}$

$\begin{array}{llll}0.33 & 0.11 & 1.66 & 0.17\end{array}$

$\begin{array}{ccccc}-0.61 & 0.51 & -0.32 & -1.19 & 0.24 \\ -2.10 & 1.22 & -0.22 & -1.71 & 0.09 \\ -0.003 & 0.003 & -0.15 & -0.98 & 0.33 \\ 2.37 & 1.72 & 0.34 & 1.38 & 0.17\end{array}$

$\begin{array}{llll}0.18 & 0.03 & 0.45 & 0.78\end{array}$

$\begin{array}{ccccc}-0.01 & 0.48 & -0.01 & -0.02 & 0.98 \\ 0.08 & 1.16 & 0.01 & 0.07 & 0.95\end{array}$


IPI
Cluster

$\underline{\text { DEPS }}$

Awake

Puff Number

Puff Duration

IPI

Cluster

Calm

Puff Number

Puff Duration

IPI

Cluster

Concentrate

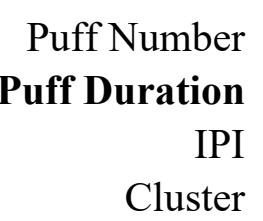

Dizzy

$$
\begin{array}{r}
\text { Puff Number } \\
\text { Puff Duration } \\
\text { IPI } \\
\text { Cluster }
\end{array}
$$

$\begin{array}{ccccc}-0.004 & 0.003 & -0.20 & -1.20 & 0.23 \\ -0.30 & 1.63 & -0.05 & -0.19 & 0.85\end{array}$

$\begin{array}{llll}0.40 & 0.16 & 2.61 & 0.05\end{array}$

$\begin{array}{ccccc}-0.88 & 0.90 & -0.26 & -0.99 & 0.33 \\ -3.79 & 2.15 & 0.22 & -1.76 & 0.08 \\ -0.01 & 0.006 & -0.29 & -1.89 & 0.06 \\ 3.85 & 3.02 & 0.31 & 1.27 & 0.21\end{array}$

$\begin{array}{llll}0.17 & 0.03 & 0.39 & 0.82\end{array}$

$\begin{array}{ccccc}-0.93 & 1.21 & -0.22 & -0.77 & 0.44 \\ 2.37 & 2.89 & 0.22 & 0.82 & 0.42 \\ -0.005 & 0.008 & -0.11 & -0.65 & 0.52 \\ 1.40 & 4.06 & 0.09 & 0.35 & 0.73\end{array}$

$\begin{array}{llll}0.60 & 0.36 & 7.64 & <.001\end{array}$

$\begin{array}{ccccc}-0.77 & 0.96 & -0.18 & -0.80 & 0.43 \\ \mathbf{- 1 0 . 0 8} & \mathbf{2 . 2 9} & \mathbf{- 0 . 4 9} & \mathbf{- 4 . 4 0} & <.001 \\ -0.008 & 0.006 & -0.16 & -1.22 & 0.23 \\ 5.38 & 3.21 & 0.35 & 1.68 & 0.10\end{array}$

$\begin{array}{llll}0.15 & 0.02 & 0.3 & 0.88\end{array}$

$\begin{array}{ccccc}-1.69 & 1.68 & -0.28 & -1.01 & 0.32 \\ -0.59 & 4.02 & -0.02 & -0.15 & 0.88 \\ -0.007 & 0.01 & -0.10 & -0.60 & 0.55 \\ 3.32 & 5.64 & 0.15 & 0.59 & 0.56\end{array}$




\begin{tabular}{|c|c|c|c|c|c|c|c|c|c|c|}
\hline Pleasant & & 0.42 & 0.18 & 2.97 & 0.03 & & & & & \\
\hline & Puff Number & & & & & 1.10 & 1.08 & 0.26 & 1.02 & 0.32 \\
\hline & Puff Duration & & & & & -3.61 & 2.60 & -0.17 & -1.39 & 0.17 \\
\hline & IPI & & & & & 0.01 & 0.007 & 0.26 & 1.72 & 0.09 \\
\hline & Cluster & & & & & 2.88 & 3.64 & 0.19 & 0.79 & 0.43 \\
\hline Reduced Hu & nger & 0.48 & 0.23 & 4.13 & 0.005 & & & & & \\
\hline & Puff Number & & & & & -1.70 & 1.04 & -0.40 & -1.63 & 0.11 \\
\hline & Puff Duration & & & & & -7.30 & 2.49 & -0.35 & -2.93 & 0.005 \\
\hline & IPI & & & & & -0.014 & 0.007 & -0.29 & -2.03 & 0.047 \\
\hline & Cluster & & & & & 5.54 & 3.50 & 0.36 & 1.59 & 0.12 \\
\hline Satisfy & & 0.42 & 0.18 & 2.91 & 0.03 & & & & & \\
\hline & Puff Number & & & & & 1.05 & 1.06 & 0.26 & 0.99 & 0.33 \\
\hline & Puff Duration & & & & & -2.51 & 2.54 & -0.12 & -0.99 & 0.33 \\
\hline & IPI & & & & & 0.01 & 0.007 & 0.25 & 1.67 & 0.10 \\
\hline & Cluster & & & & & 3.27 & 3.56 & 0.22 & 0.92 & 0.36 \\
\hline Sick & & 0.32 & 0.10 & 1.51 & 0.21 & & & & & \\
\hline & Puff Number & & & & & -1.21 & 1.25 & -0.26 & -0.97 & 0.34 \\
\hline & Puff Duration & & & & & 4.02 & 3.00 & 0.18 & 1.34 & 0.19 \\
\hline & IPI & & & & & -0.003 & 0.008 & -0.05 & -0.33 & 0.74 \\
\hline & Cluster & & & & & -0.36 & 4.20 & -0.02 & -0.09 & 0.93 \\
\hline Taste Good & & 0.41 & 0.16 & 2.66 & 0.04 & & & & & \\
\hline & Puff Number & & & & & 1.39 & 1.34 & 0.27 & 1.03 & 0.31 \\
\hline & Puff Duration & & & & & -4.95 & 3.22 & -0.19 & -1.54 & 0.13 \\
\hline & IPI & & & & & 0.014 & 0.009 & 0.234 & 1.55 & 0.13 \\
\hline & Cluster & & & & & 2.71 & 4.52 & 0.14 & 0.60 & 0.55 \\
\hline
\end{tabular}




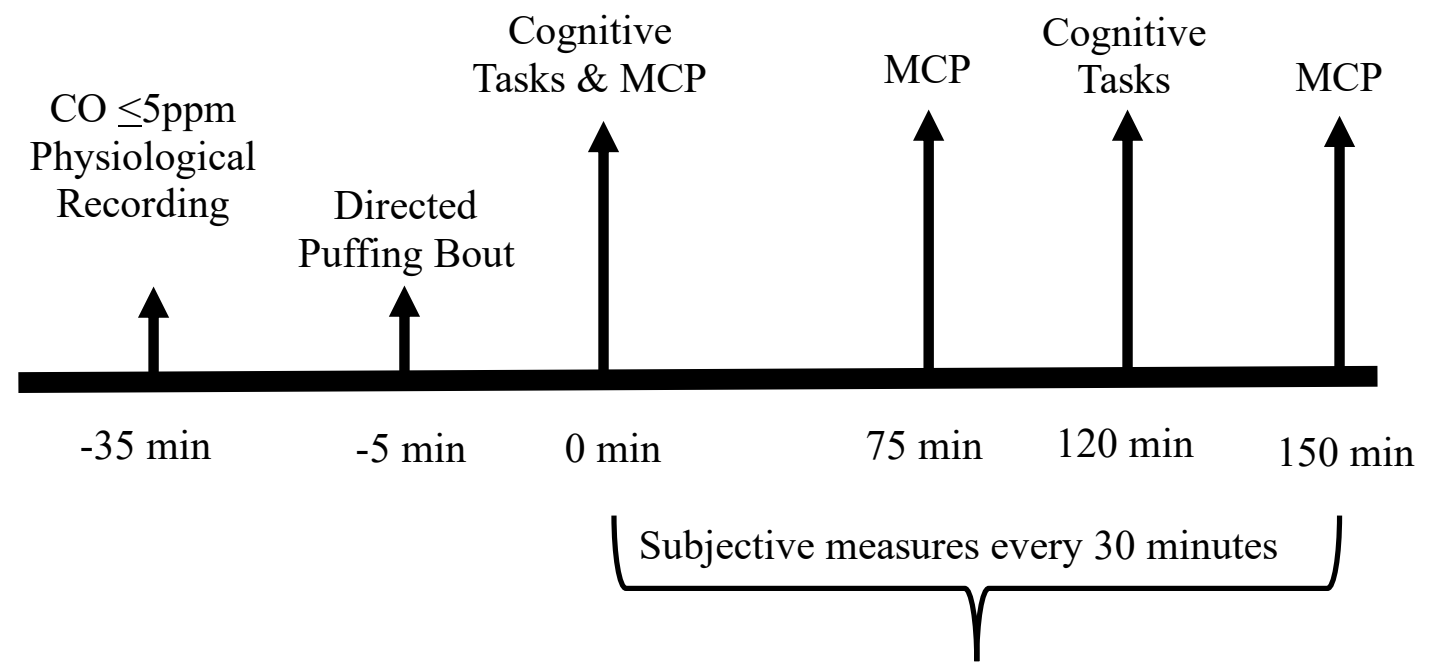

ECIG abstinence or ad libitum use

Figure 1. Session procedure diagram. 
Panel A: Correctly Identified

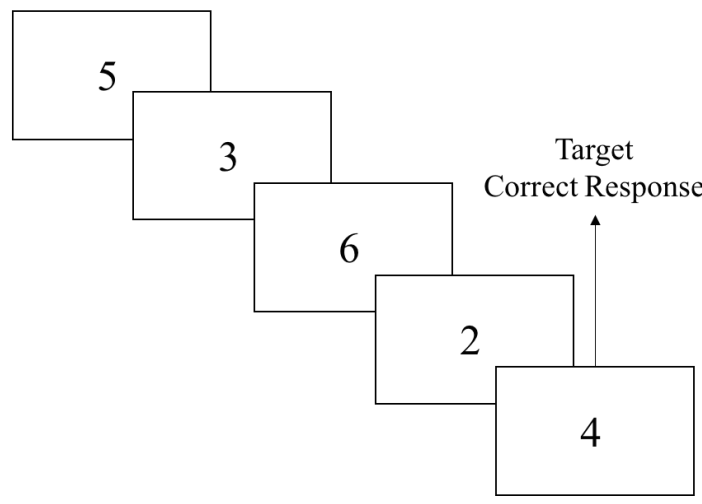

Panel B: Incorrect, False Positive

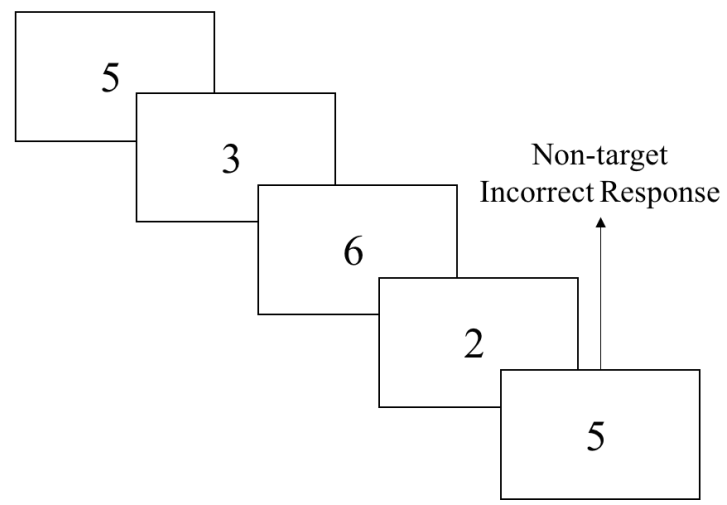

Figure 2. Rapid Visual Information Processing task diagram. Panel A demonstrates a target, as demonstrated by the presentation of three consecutive even (or odd) digits. A response to the target is defined as a correct response. Panel B demonstrates a non-target, as demonstrated by the lack of presentation of three consecutive even or odd digits. A response on the non-target is defined as an incorrect response. 


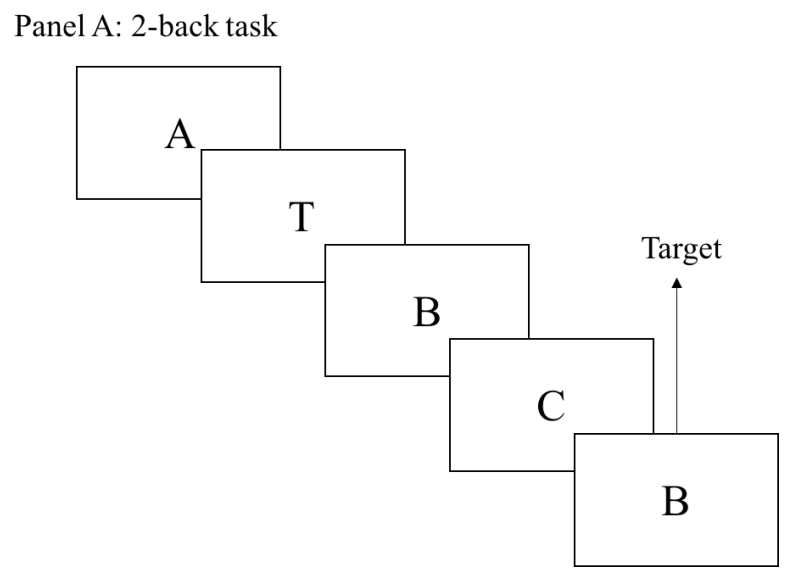

Panel B: 3-back task

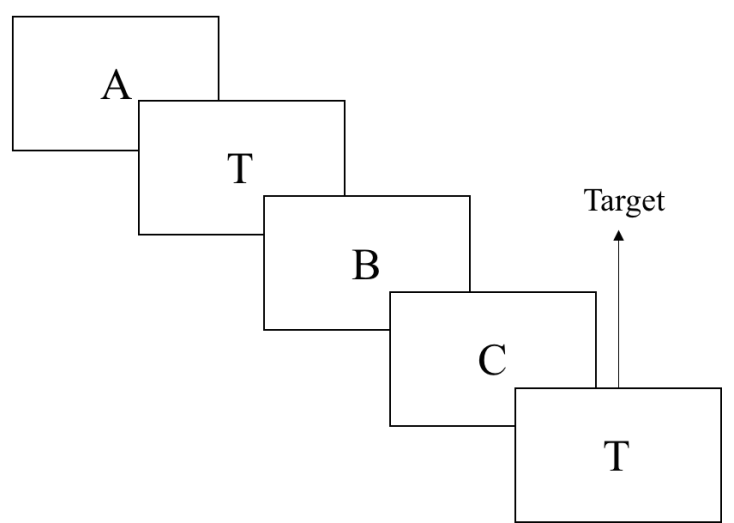

Figure 3. $\mathrm{N}$-back task diagram. Panel A demonstrates the 2-back condition. A target is a stimulus that is identical to the stimulus presented two letters prior. A "yes" response to the target is considered a hit, while a "no" response is an incorrect response. Panel B demonstrates the 3-back condition. A target is a stimulus that is identical to the stimulus presented three letters prior. A "yes" response to the target is considered a hit, while a "no" response is an incorrect response. 
Panel A: Congruent Go Target

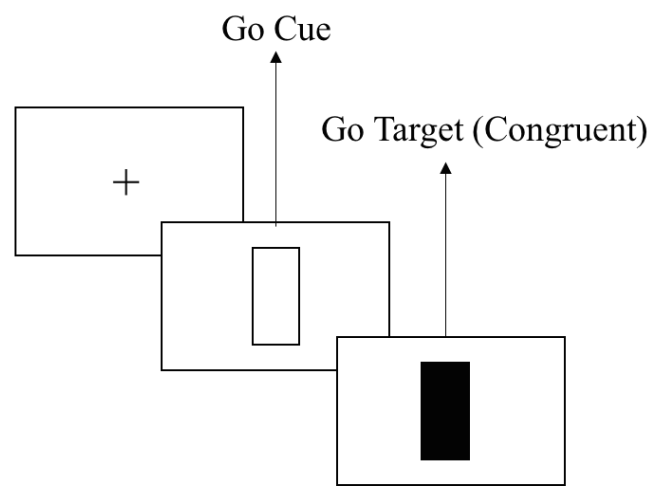

Panel C: Congruent No-Go Target

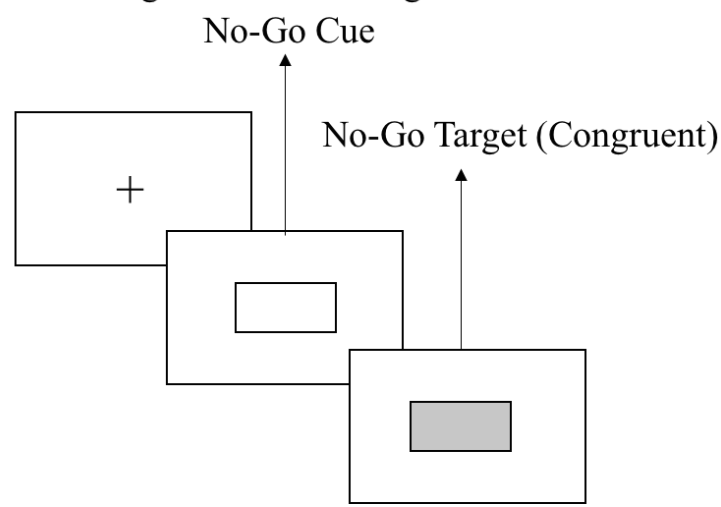

Panel B: Incongruent Go Target

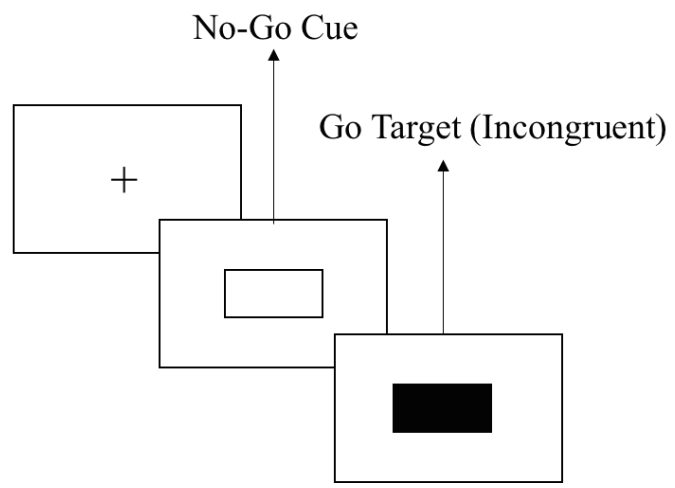

Panel D: Incongruent No-Go Target

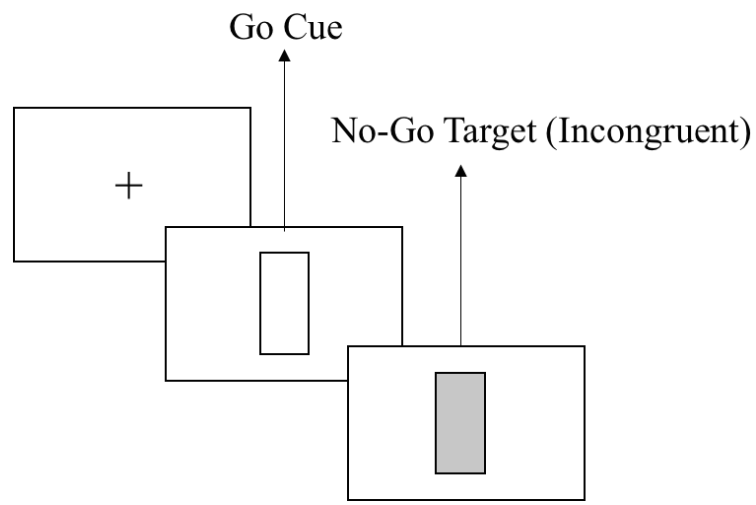

Figure 4. Cued Go/No-Go task diagram. For the purposes of this figure, green rectangles are represented in black and blue rectangles are represented in gray. Panel A demonstrates a congruent Go target. A Go cue is presented as a vertical rectangle. The go target is represented by the black (green) fill. The target is congruent because the cue and the target indicate it is a Go trial. Panel B demonstrates an incongruent Go target. A No-Go cue is presnted as a horizontal rectangle. The Go target is represented by the black (green) fill. The target is incongruent because the cue indicates a No-Go trial, but the target requires a Go response. Panel C demonstrates a congruent No-Go target. A No-Go cue is presented as a horizontal rectangle. The No-Go target is represented by the gray (blue) fill. The target is congruent because the cue and the target indicate it is as No-Go trial. Panel D demonstrates an incongruent No-Go target. A Go cue is presented as a vertical rectangle. A No-Go target is represented by the gray (blue) fill. The 
target is incongruent because the cue indicates a Go trial, but the target requires a No-Go response. 


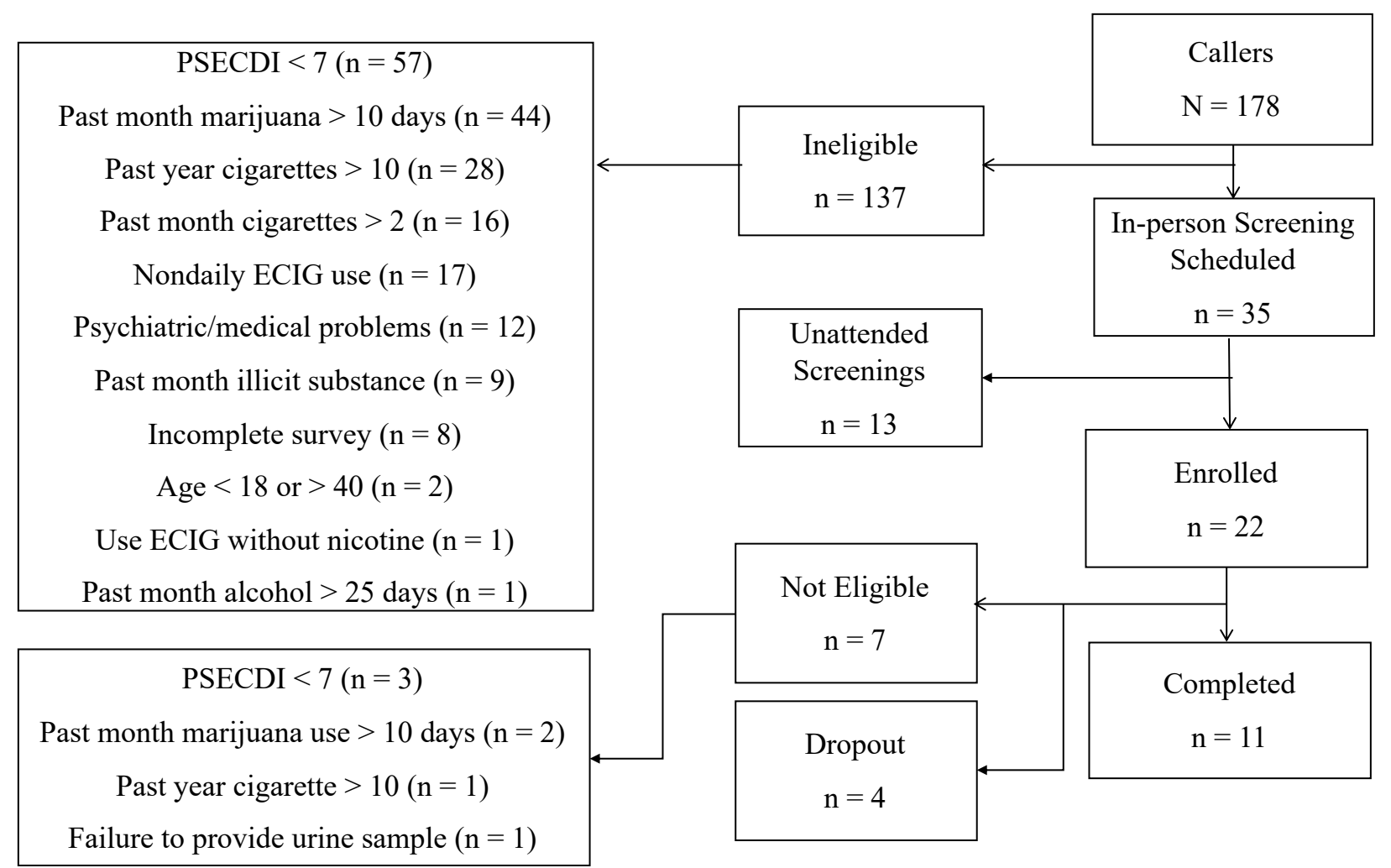

Figure 5. Diagram of recruitment, enrollment, reasons for ineligibility, and number of completers. Ineligible callers may have been ineligible for multiple reasons. 


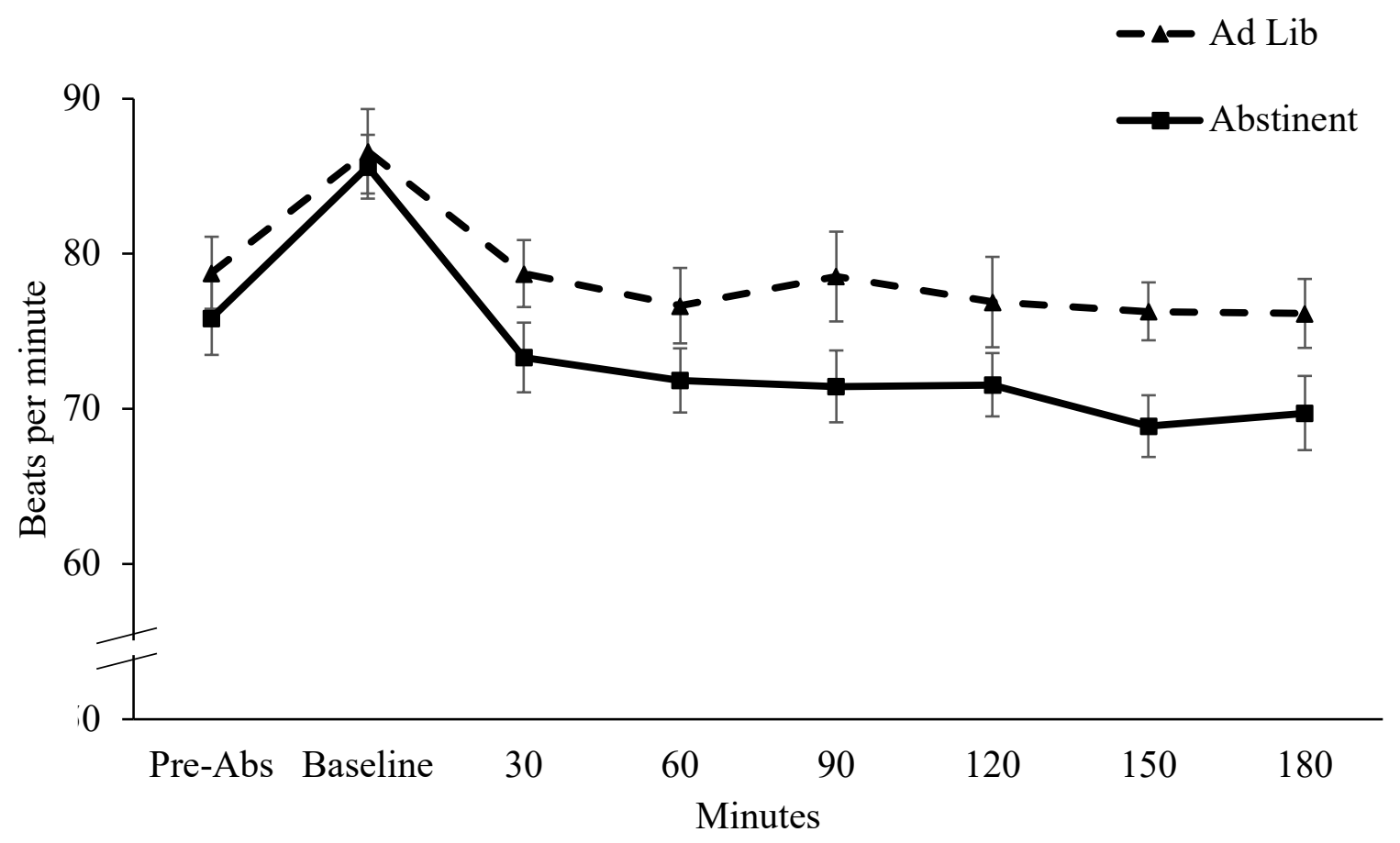

Figure 6. Mean heart rate during 180 minutes of ad lib ECIG use or ECIG abstinence. The error bars represent standard error of the mean. 


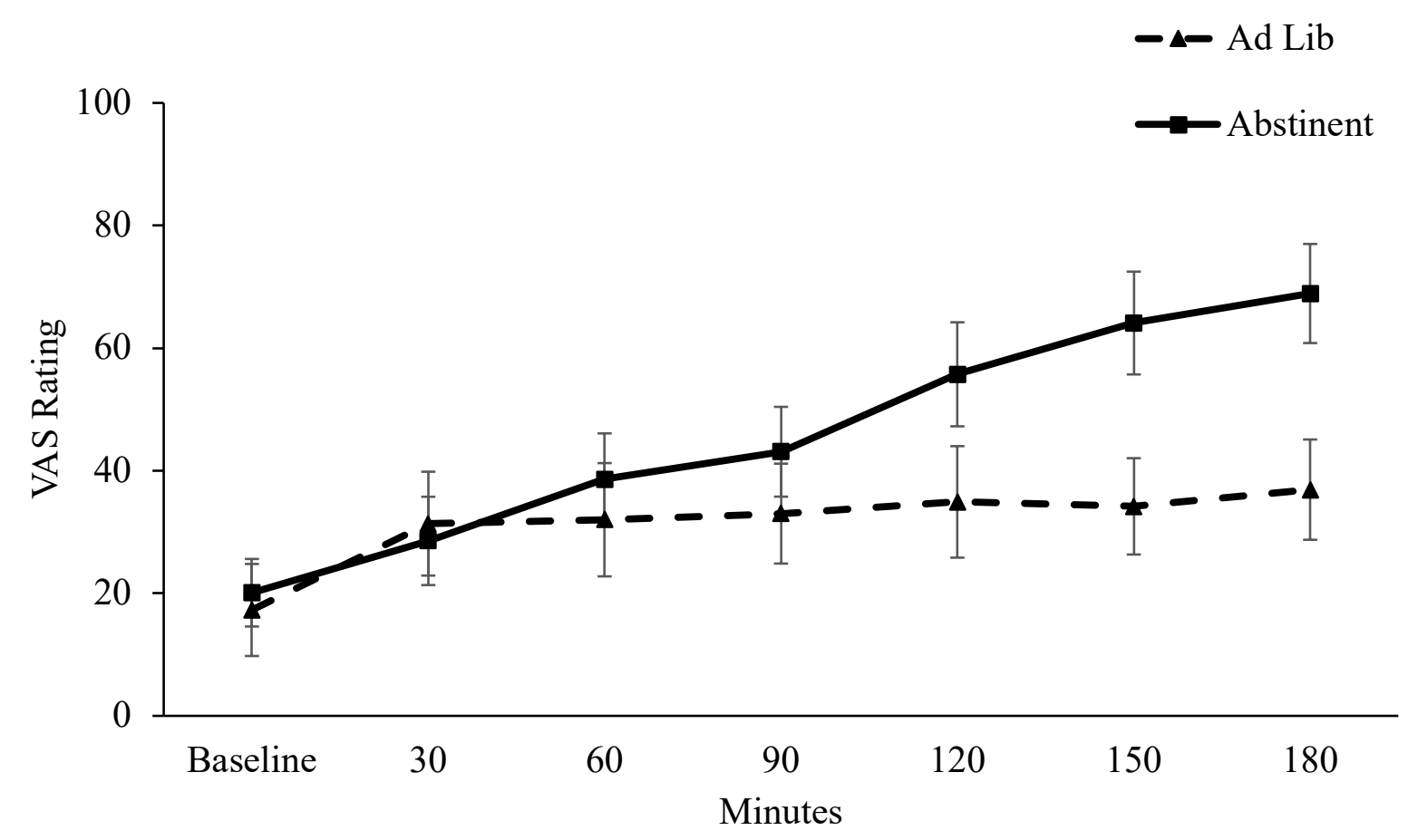

Figure 7. Mean ratings for MNWS craving during 180 minutes of ad lib ECIG use or ECIG abstinence. The error bars represent standard error of the mean. 


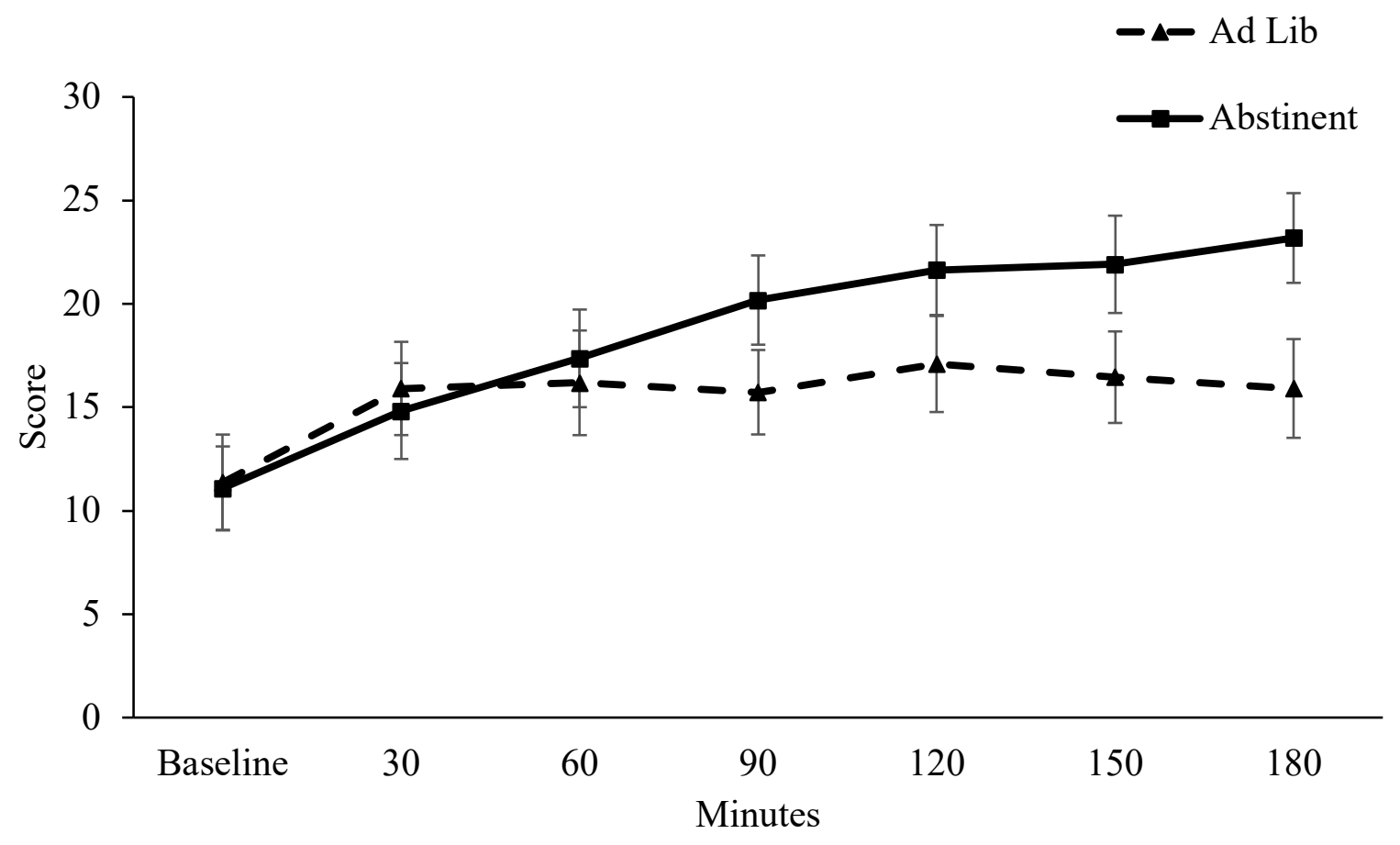

Figure 8. Mean ratings for QSU-Brief Factor 1 (intention to smoke) during 180 minutes of ad lib ECIG use or ECIG abstinence. The error bars represent standard error of the mean. 


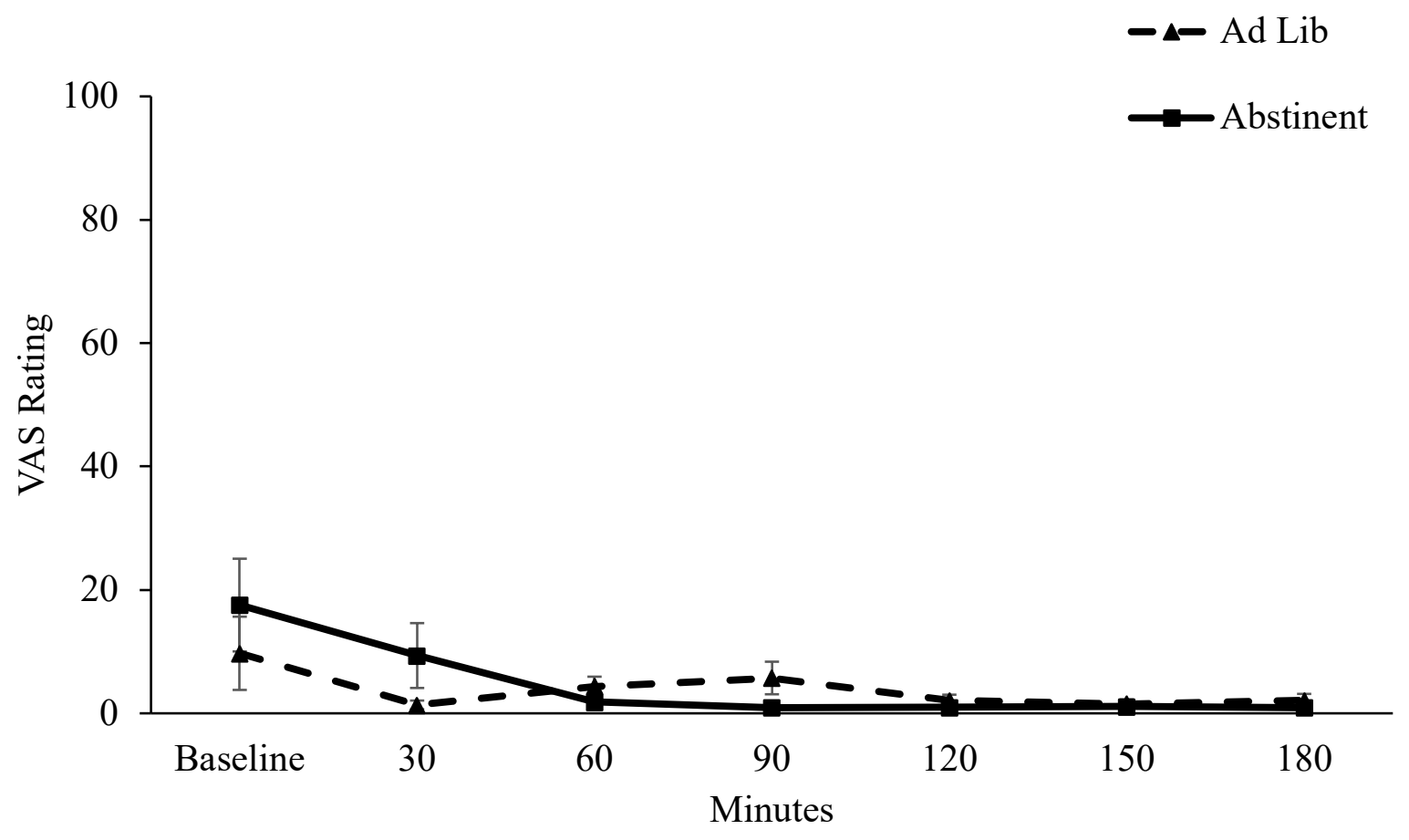

Figure 9. Mean ratings for DENS weak during 180 minutes of ad lib ECIG use or ECIG abstinence. The error bars represent standard error of the mean. 


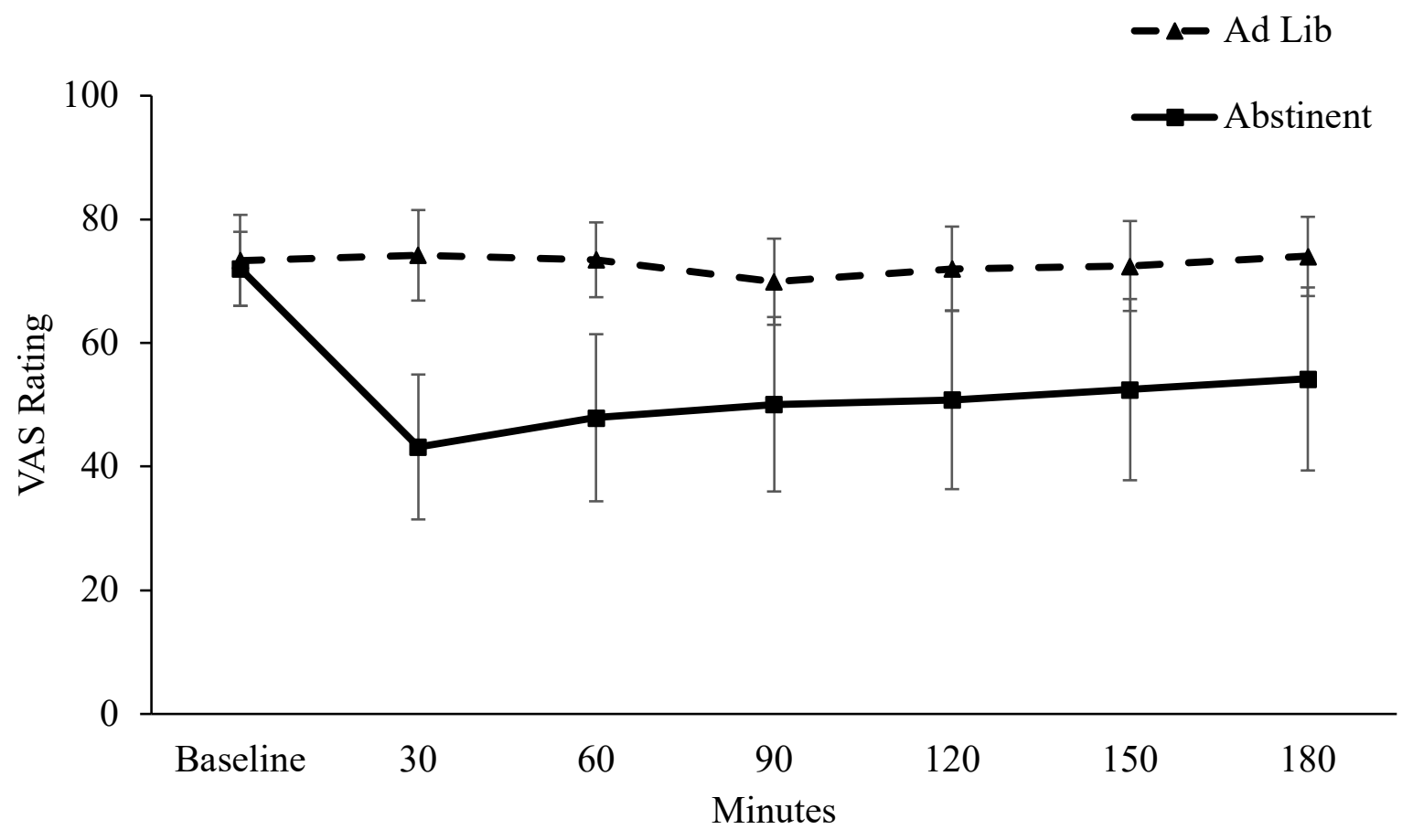

Figure 10. Mean ratings for DEPS pleasant during 180 minutes of ad lib ECIG use or ECIG abstinence. The error bars represent standard error of the mean. 


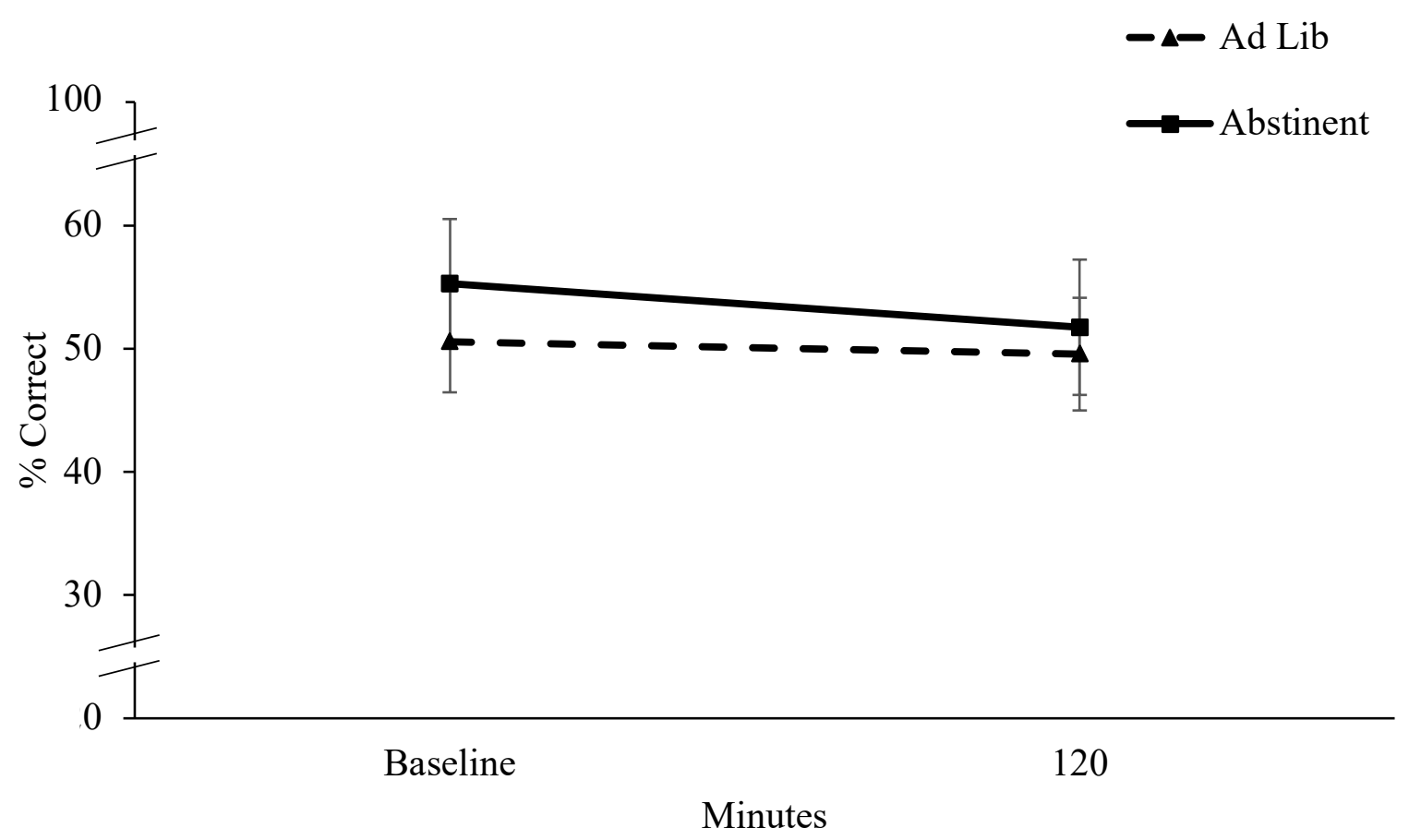

Figure 11. Mean RVIP percent correct for ad lib and abstinent conditions at baseline and 120 minutes. The error bars represent standard error of the mean. 


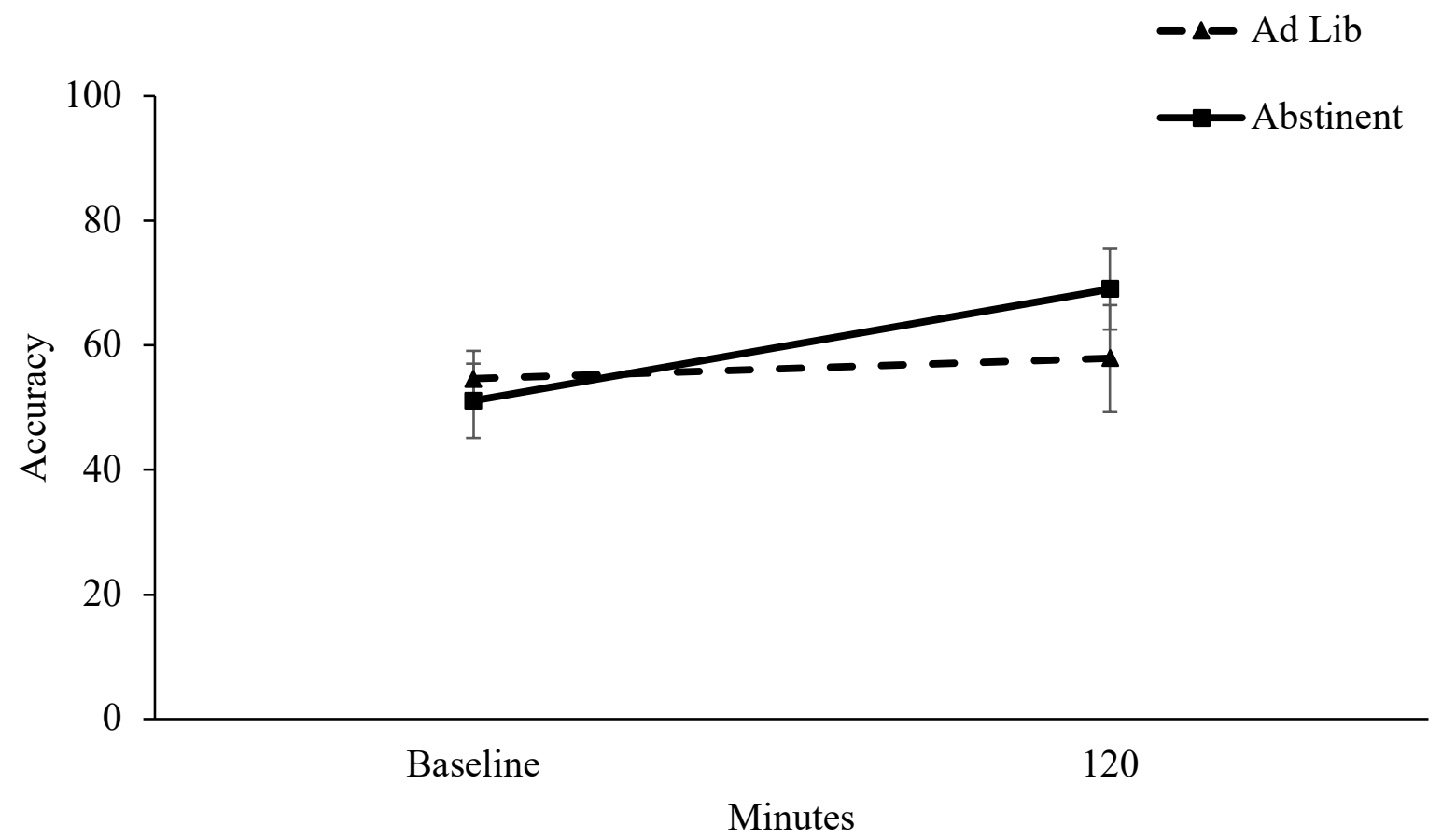

Figure 12. Mean 3-back accuracy for ad lib and abstinent conditions at baseline and 120 minutes. The error bars represent standard error of the mean. 


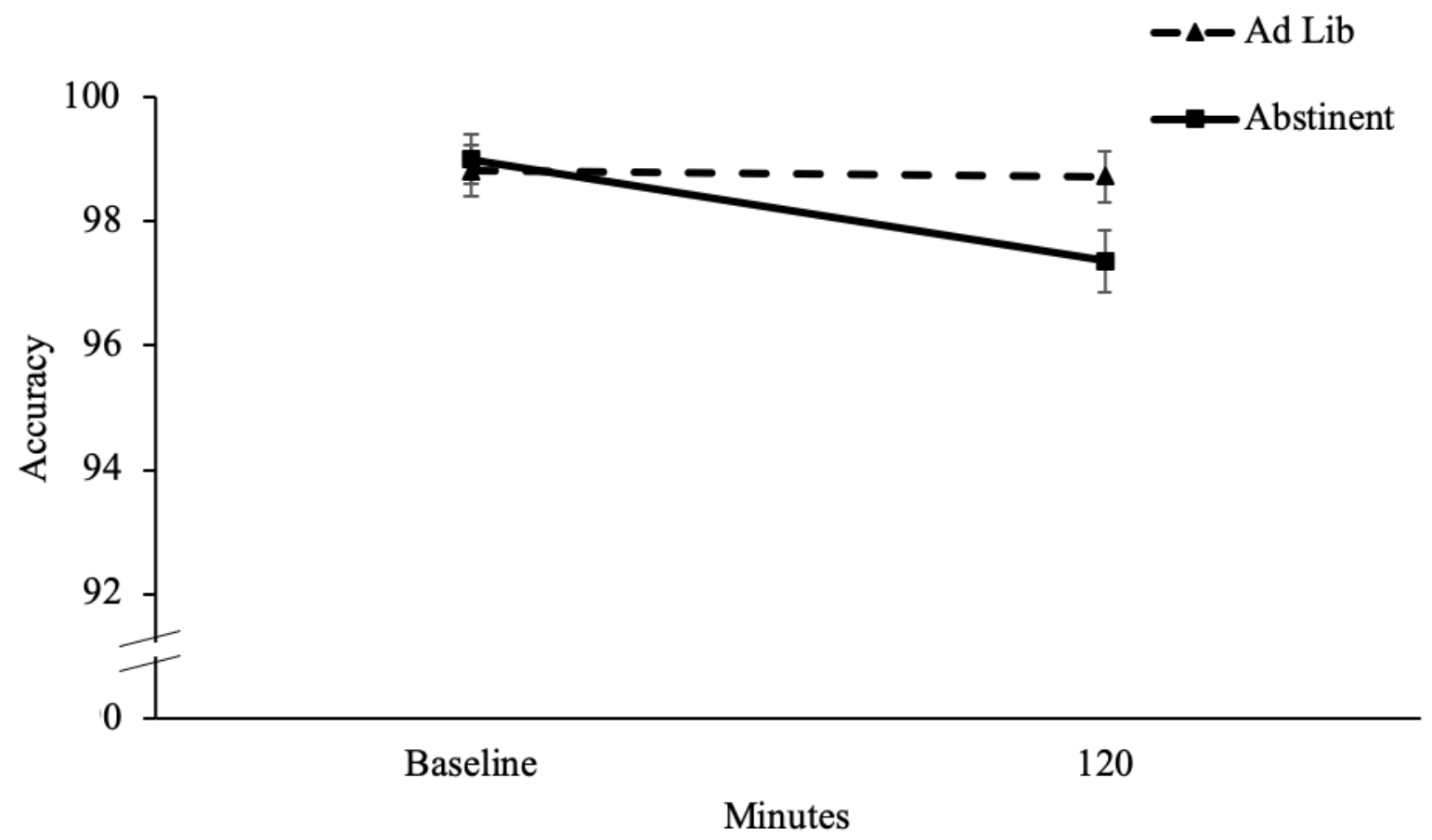

Figure 13. Mean No-Go incongruent accuracy for $a d$ lib and abstinent conditions at baseline and 120 minutes. The error bars represent standard error of the mean. 
501 Puffs: Time 0 - $60 \mathrm{~min}$

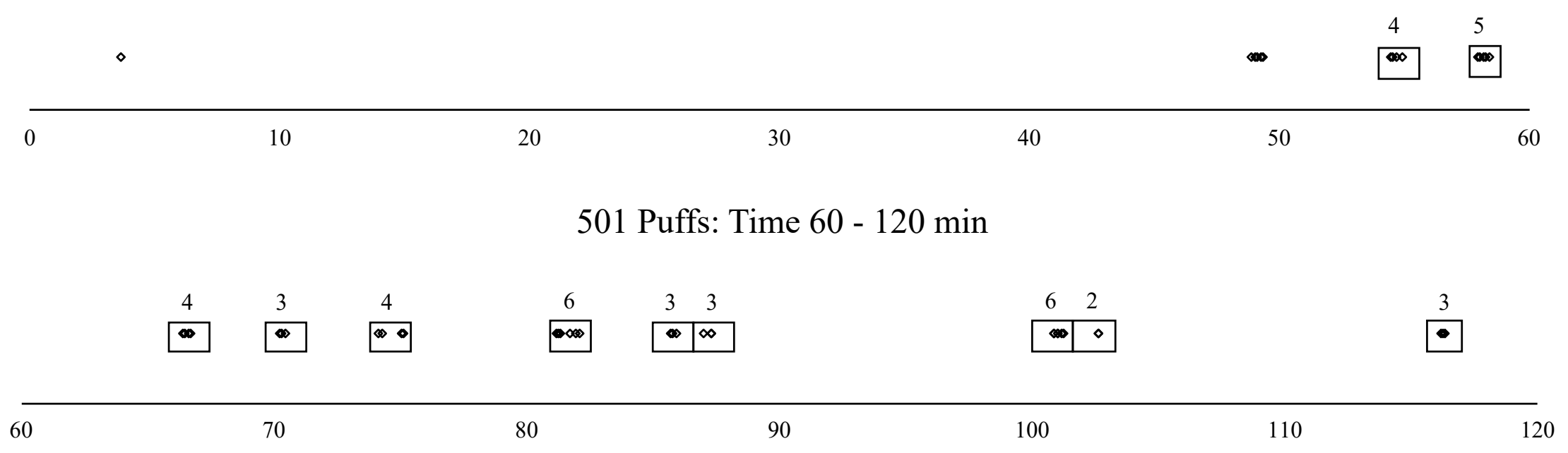

501 Puffs: Time $120-180$ min

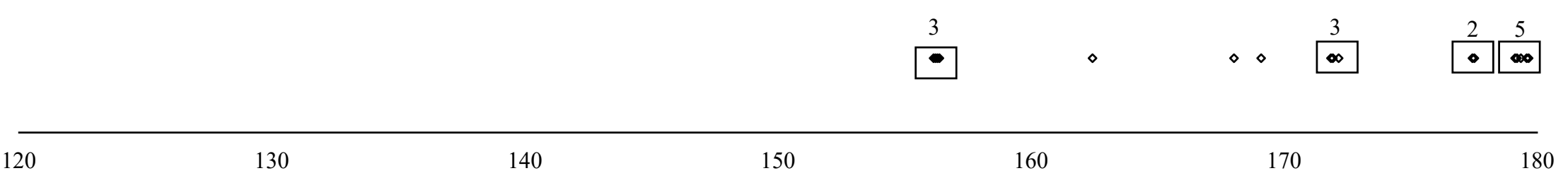

Figure 14. Puffing patterns in the ad lib condition for participant 501. Participant 501 used a $3^{\text {rd }}$ generation mod at 50 watts, with a berry-flavored liquid with $3 \mathrm{mg} / \mathrm{ml}$ nicotine. Boxes represent a puff cluster with the number above indicating the number of puffs in the cluster. 
507 Puffs: Time 0 - 60 minutes

20

\begin{tabular}{llllll}
\hline 0 & 10 & 20 & 30 & 40 & 50
\end{tabular}

507 Puffs: Time 60 - 120 minutes

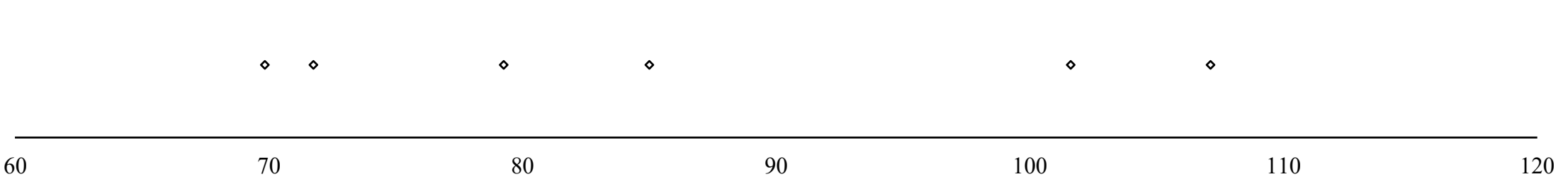

507 Puffs: Time 120 - 180 minutes

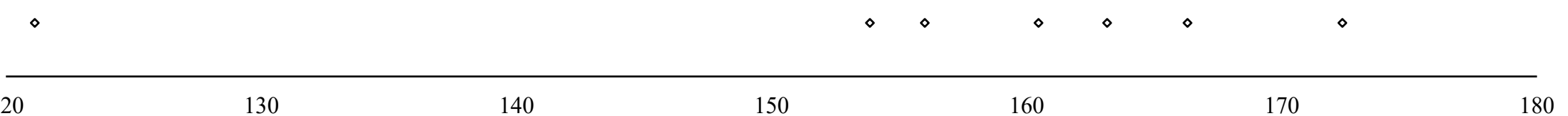

Figure 15. Puffing patterns in the ad lib condition for participant 507. Participant 507 used a $4^{\text {th }}$ generation pod (Juul) at 8 watts, with a mint-flavored liquid with $59 \mathrm{mg} / \mathrm{ml}$ nicotine. Boxes represent a puff cluster with the number above indicating the number of puffs in the cluster. 


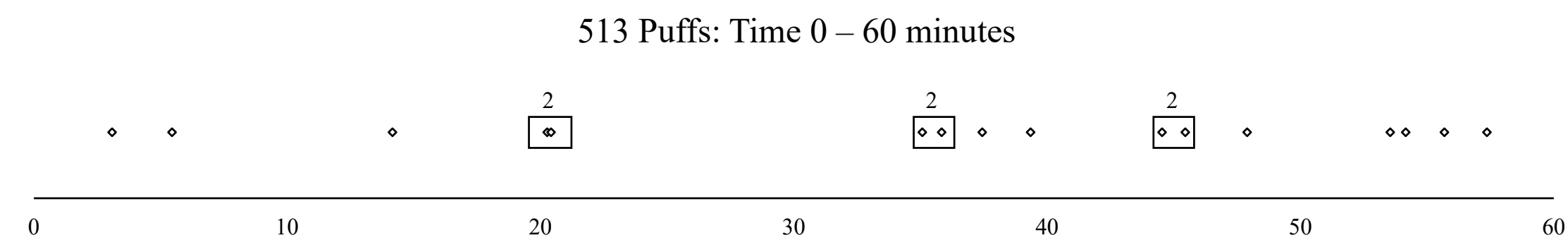

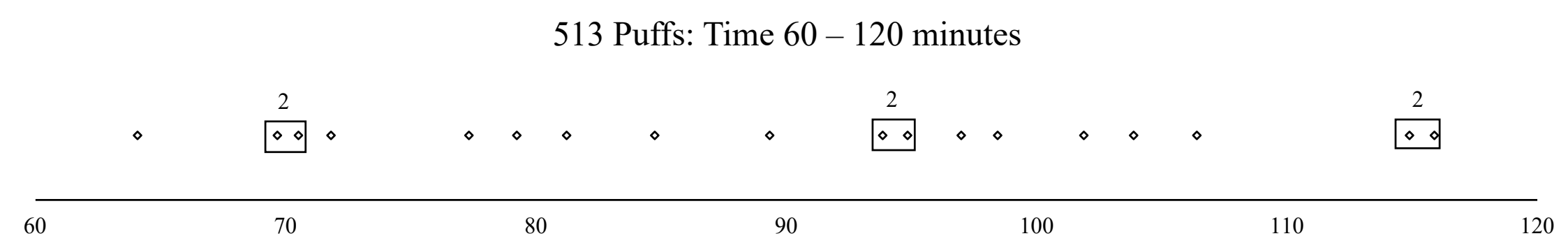

513 Puffs: Time $120-180$ minutes

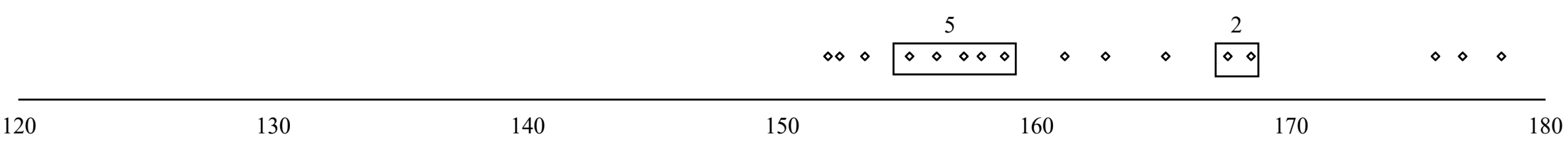

Figure 16. Puffing patterns in the ad lib condition for participant 513. Participant 513 used a $4^{\text {th }}$ generation pod (Juul) at 8 watts, with a mint-flavored liquid with $59 \mathrm{mg} / \mathrm{ml}$ nicotine. Boxes represent a puff cluster with the number above indicating the number of puffs in the cluster. 


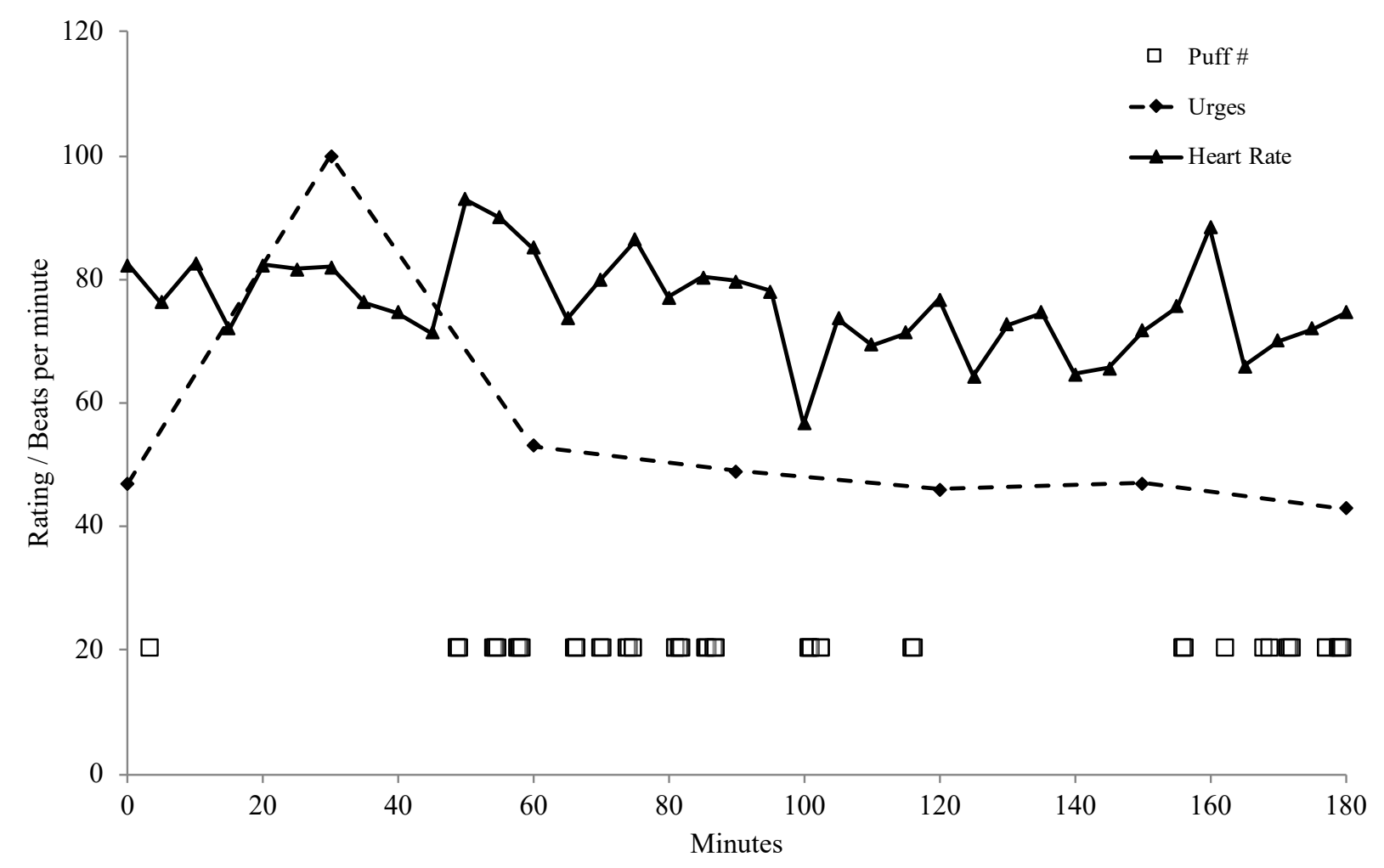

Figure 17. Puffing patterns, heart rate, and MNWS urges for participant 501 in the ad lib condition. Participant 501 used a $3^{\text {rd }}$ generation mod at 50 watts, with a berry-flavored liquid with $3 \mathrm{mg} / \mathrm{ml}$ nicotine. 


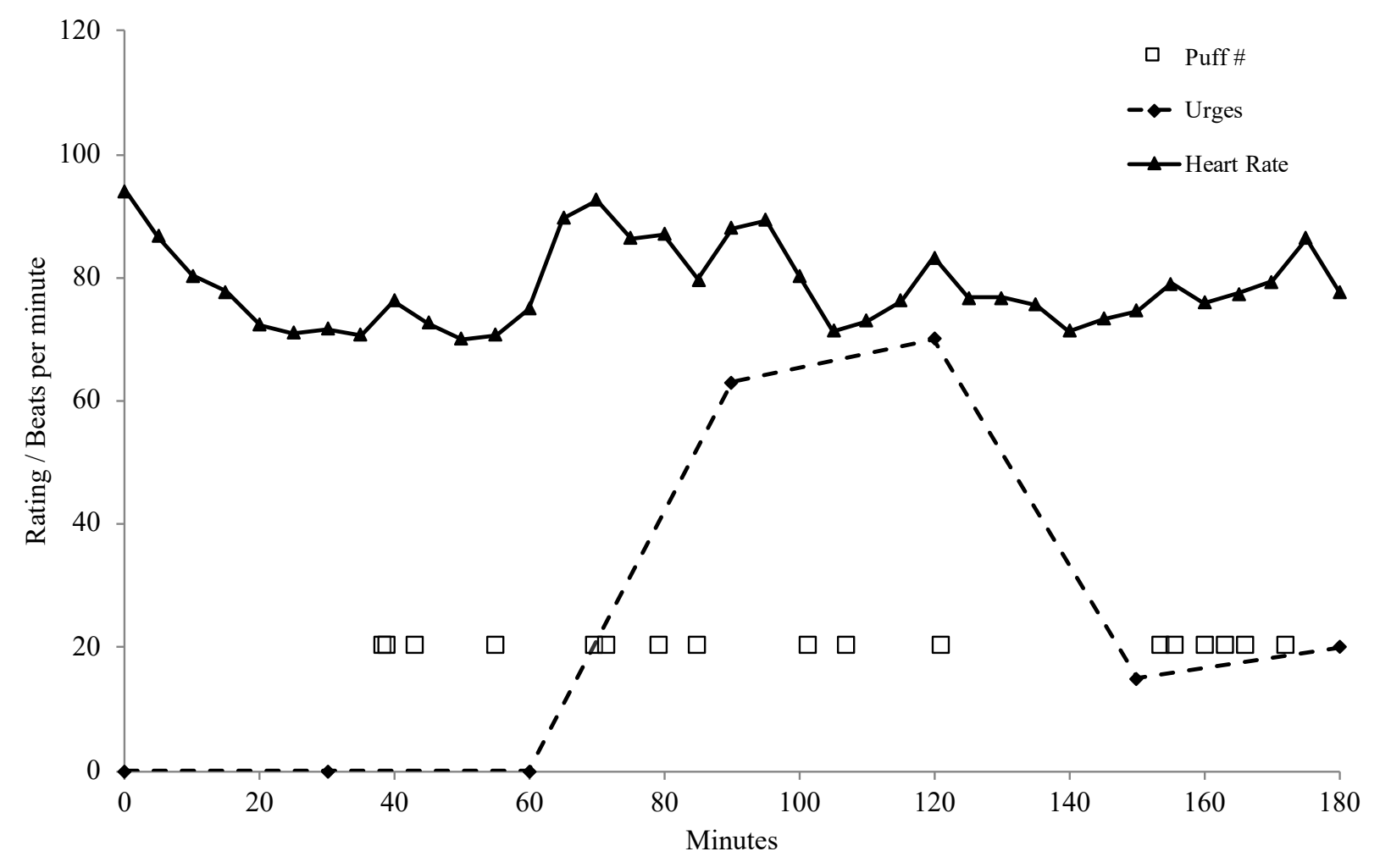

Figure 18. Puffing patterns, heart rate, and MNWS urges for participant 507 in the ad lib condition. Participant 507 used a $4^{\text {th }}$ generation pod (Juul) at 8 watts, with a mint-flavored liquid with $59 \mathrm{mg} / \mathrm{ml}$ nicotine. 


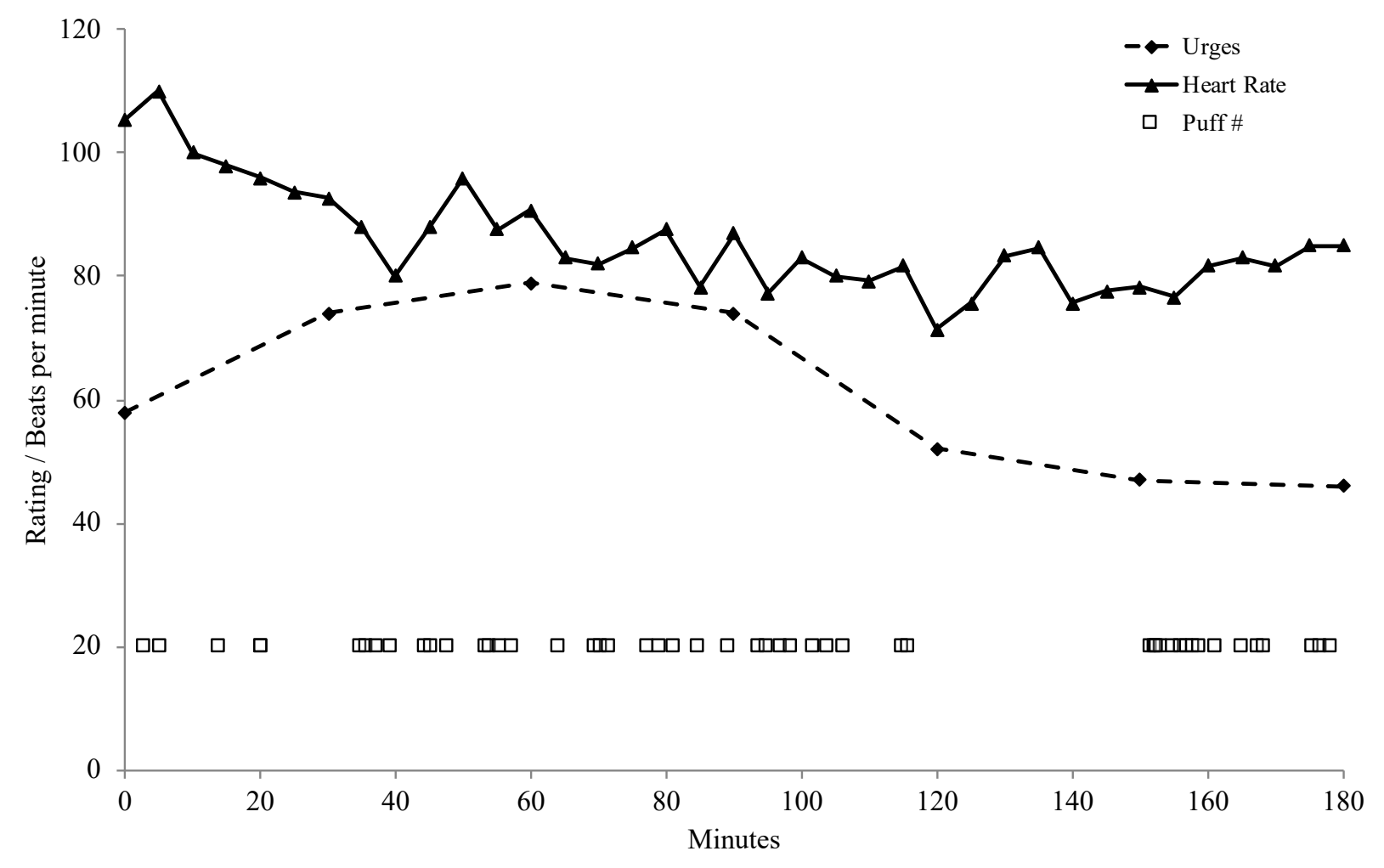

Figure 19. Puffing patterns, heart rate, and MNWS urges for participant 513 in the ad lib condition. Participant 513 used a $4^{\text {th }}$ generation pod (Juul) at 8 watts, with a mint-flavored liquid with $59 \mathrm{mg} / \mathrm{ml}$ nicotine. 


\author{
Appendix A \\ Telephone Screening Questionnaire
}

Date:

Interviewer:

Interviewer: "I would like to ask you some questions about yourself and your health status as well as your use of tobacco, alcohol, and other drugs. The purpose of these questions is to determine whether or not you are eligible to participate in the study that I just described to you. All of your responses are confidential. You are not required to answer any question and you may stop this interview at any time. May I begin the questions?"

Document caller's response by circling either: Yes or No

\title{
If Yes: begin form. If No: thank the caller and stop the interview.
}

How did you hear about us/our study?

\section{Personal Information:}

1. "What is your first name?"

2. "What is a phone number/email at which you can be contacted?"

3. "If we call and you are not available, may we leave a message?" Yes or No

4. "What is your date of birth?"

\section{General health status:}

1. "Are you currently under a doctor's care for a medical condition?" Yes or No

If Yes: "Please describe the concern or problem":

2. "Do you have any chronic health concerns or problems?" Yes or No

If Yes: "Please describe the condition":

3. "Are you taking any prescription or over-the-counter medications?" Yes or No

If Yes: "Please identify the medication":

4. Have you been diagnosed with any psychiatric conditions like bipolar disorder or schizophrenia?

Yes or No

If Yes: "Please describe the condition":

\section{For women only:}


6. "Are you currently pregnant?"

7. "Are you currently breast-feeding a child?"

\section{Cigarette Use:}

1. "Have you ever smoked tobacco cigarettes?"

2. "Do you currently smoke tobacco cigarettes?"

3. "How many cigarettes have you smoked in your lifetime?"

4. "How many cigarettes have you smoked in the past year?"

5. "How any cigarettes have you smoked in the past month?"
Yes or No

Yes or No

\section{Electronic Cigarette Use:}

1. "Do you currently use an electronic cigarette?" Yes or No

If No: Skip to the section on other tobacco use

2. "What model/brand of ECIG do you own?

a) If own multiple, which do you use most frequently?

3. "What nicotine concentration of e-liquid do you use?"

$(m g / m l)$

4. "What flavor of e-liquid do you use?"

5. "On average, how many days per week do you use an ECIG?"

6. "How much e-liquid/How many cartridges do you use per day? ( $m l$ or \#)

[Guide to report one number that best represents their average/day; do not provide a range]

7. "For how long have you used that amount of product?" (months / years)

8. What ratio of propylene glycol : vegetable glycerin do you use? $\mathrm{vg})$

$(\%$ pg : \%

9. At what wattage do you typically vape?

(Watts)

[Watts $=($ Volts/Resistance $) \times$ Volts; Voltage refers to battery, resistance refers to atomizer $)$

\section{ECIG Dependence:}


1. How many times per day do you usually use your electronic cigarette? (assume that one time consists of around 15 puffs or lasts around 10 minutes)
a. $0-4$
b. $5-9$
c. $10-14$
d. $15-19$
e. $20-20$
f. $30+$

2. How soon after you wake up do you first use your electronic cigarette?
a. $0-5 \mathrm{~min}$
b. $6-15 \mathrm{~min}$
c. $16-30 \mathrm{~min}$
d. $31-60 \mathrm{~min}$
e. $121+$ $\min$

3. Do you sometimes awaken at night to use your electronic cigarette?
a. Yes
b. No

4. If yes, how many nights per week do you typically awaken to use your electronic cigarette?
a. $0-1$ night
b. 2-3 nights
c. $4+$ nights

5. Do you use an electronic cigarette now because it is really hard to quit?
a. Yes
b. No

6. Do you ever have strong cravings to use an electronic cigarette?
a. Yes
b. No

7. Over the past week, how strong have the urges to use an electronic cigarette been?
a. None/Slight
b. Moderate/Strong
c. Very/Extremely Strong

8. Is it hard to keep from using an electronic cigarette in places where you're not supposed to?
a. Yes
b. No

9. Did you feel more irritable because you couldn't use an electronic cigarette?
a. Yes
b. No

10. Did you feel nervous, restless, or anxious because you couldn't use an electronic cigarette
a. Yes
b. No

\section{Other Tobacco Use:}

1. "Have you used any of the following other nicotine/tobacco products in the past month?" 


\begin{tabular}{|c|c|c|c|}
\hline & & & $\begin{array}{c}\text { If 'yes', estimate how many days } \\
\text { you have used this product in the } \\
\text { past month? }\end{array}$ \\
\hline $\begin{array}{c}\text { Large cigars } \\
\begin{array}{c}\text { Cigarillos (e.g., Black \& Milds) } \\
\text { or small cigars }\end{array}\end{array}$ & No & Yes & Yes \\
\hline $\begin{array}{c}\text { Waterpipe (a.k.a. hookah or } \\
\text { shisha) }\end{array}$ & No & Yes & \\
\hline $\begin{array}{c}\text { Smokeless tobacco } \\
\text { (snuff/dip/chew/snus) }\end{array}$ & No & Yes & \\
\hline $\begin{array}{c}\text { Cigarettes } \\
\text { Other: }\end{array}$ & No & Yes & \\
\hline
\end{tabular}

\section{Smoking Cessation History:}

1. "Are you currently using any nicotine replacement products?" $\quad Y e s \quad$ or No (e.g., patch, gum, inhaler, nasal spray)

2. "Are you currently using any prescription medications for cessation?" Yes or No (e.g., Chantix, Zyban, etc.)

\section{Illicit Drug Use:}

1. "Have you used any of the following other drugs for recreational purposes in the past month?"

\begin{tabular}{|c|c|c|c|}
\hline & & & $\begin{array}{c}\text { If 'yes', estimate how many days } \\
\text { you have used this product in the } \\
\text { past month? }\end{array}$ \\
\hline Alcohol & No & Yes & \\
\hline Marijuana / Spice / K2 & No & Yes & \\
\hline $\begin{array}{c}\text { Stimulants (e.g., cocaine, } \\
\text { amphetamine, etc.) }\end{array}$ & No & Yes & \\
\hline $\begin{array}{c}\text { Opiates (e.g., heroin, oxycodone, } \\
\text { etc.) }\end{array}$ & No & Yes & \\
\hline Other: & No & Yes & \\
\hline
\end{tabular}


Interviewer: "Thank you for responding to these questions. I need to pass on your responses to the principal investigator who will then determine whether or not you are eligible to participate in a study. If you are eligible, someone will contact you within approximately one week. If you are not eligible for this study, then you will not be contacted."

[If respondent does not have a phone, they can call us back in a few days] 


\section{Appendix B}

Demographic Information

Participant ID:

Today's Date :

Age

Years:

Date of birth

\section{Ethnicity}
o Hispanic or Latino
o Not Hispanic or Latino

Race

o American Indian/Alaskan Native o Asian o White

o Native Hawaiian or other Pacific Islander

o Black or African American o More than 1 race o Other/Unknown

\section{Gender}
o Male
o Female
o Other/Wish Not to Report

Marital status

o Single o Married o Separated o Divorced o Widowed

\section{Education}

Years: $\_$(For example, High school $=12$, College degree $=16$, etc.)

\section{Primary employment}

o Unemployed o Part Time (0-30 hrs/wk) o Full Time ( $>30 \mathrm{hrs} / \mathrm{wk}) \quad$ o Student 


\section{Appendix C \\ Health and Drug Use Form}

Participant ID:

Date:

\section{General health status:}

Are you under a doctor's care for a medical condition? (If yes, please describe below)

Are you taking any prescription medications? (If yes, please identify below)

Do you have any chronic health concerns or problems? (If yes, please describe below)

Have you been diagnosed with any psychiatric conditions such as schizophrenia or bipolar disorder? (If yes, please describe below)

\section{For women only:}

Are you currently pregnant? (yes or no)

Are you currently breast-feeding a child? (yes or no)

\section{Cigarette Use:}

Have you ever smoked tobacco cigarettes? (yes or no)

Do you currently smoke tobacco cigarettes? (yes or no)

How many cigarettes have you smoked in your lifetime? (\# of cigarettes)

How many cigarettes have you smoked in the past year? (\# of cigarettes)

How many cigarettes have you smoked in the past month? (\# of cigarettes)

\section{ECIG Use:}

Do you currently use an ECIG? (yes or no) if no, skip to the next section

How many days per week do you use an ECIG? (number of days)

On average, how much e-liquid/how many cartridges do you use per day? $(\mathrm{ml}$ or \#)

What nicotine concentration of e-liquid do you use? $(\mathrm{mg} / \mathrm{ml}$ or $\%)$

For how long have you been using an ECIG? (months/years) 
What model ECIG do you use most frequently?

What flavor of e-liquid do you use most frequently?

What ratio of propylene glycol : vegetable glycerin do you use? $(\% \mathrm{PG}: \% \mathrm{VG})$

At what wattage do you typically vape? (watts)

\section{ECIG Dependence}

1. How many times per day do you usually use your electronic cigarette? (assume that one time consists of around 15 puffs or lasts around 10 minutes)
a. $0-4$
b. $5-9$
c. $10-14$
d. $15-19$
e. $20-20$
f. $30+$

2. How soon after you wake up do you first use your electronic cigarette?
a. $0-5 \mathrm{~min}$
b. $6-15 \mathrm{~min}$
c. $16-30 \mathrm{~min}$
d. 31-60 min
e. $121+$ $\min$

3. Do you sometimes awaken at night to use your electronic cigarette?
a. Yes
b. No

4. If yes, how many nights per week do you typically awaken to use your electronic cigarette?
a. 0-1 night
b. 2-3 nights
c. $4+$ nights

5. Do you use an electronic cigarette now because it is really hard to quit?
a. Yes
b. No

6. Do you ever have strong cravings to use an electronic cigarette?
a. Yes
b. No

7. Over the past week, how strong have the urges to use an electronic cigarette been?
a. None/Slight
b. Moderate/Strong
c. Very/Extremely Strong

8. Is it hard to keep from using an electronic cigarette in places where you're not supposed to?
a. Yes
b. No

9. Did you feel more irritable because you couldn't use an electronic cigarette?
a. Yes
b. No

10. Did you feel nervous, restless, or anxious because you couldn't use an electronic cigarette
a. Yes
b. No 


\section{History of Quit Attempts:}

Have you ever made an attempt to quit or reduce your ECIG use? (yes or no) if no, skip to the next section

Have you made any attempts to quit or reduce your ECIG use in the last 30 days?

\section{Other Tobacco Use:}

Do you currently use any other nicotine/tobacco products? (yes or no)

Circle all products below that you have used in the past 30 days:

Cigars / cigarillos / small cigars Smokeless tobacco (snuff, dip, chew) / snus

Hookah / waterpipe Cigarettes

Nicotine gum / patch / lozenge / inhaler Other:

\section{Alcohol Use:}

Have you used alcohol in the past month? (yes or no) if no, skip to the next section

How many days out of the last 30 have you used alcohol? (number of days)

Have you ever been treated for alcohol abuse/dependence? (yes or no)

\section{Other Drug Use:}

Have you used marijuana in the past month? (yes or no) if no, skip to the next section How many days out of the last 30 have you used marijuana? (number of days)

Have you ever been treated for marijuana abuse/dependence? (yes or no)

Have you used any illegal drugs within the past month? (yes or no)

If yes, please identify which drugs and how many times in the past month: 


\section{Appendix D \\ MINI Tobacco Use Disorder Screen for ECIGs}

\section{J2: Considering your use of ECIGs, in the past 12 months:}

a. During times when you used the drug, did you end up using more than you planned when you started?

b. Did you repeatedly want to reduce or control your ECIG use? Did you try to cut down or control your ECIG use, but failed?

(If yes to either, code YES)

c. On the days that you used more ECIG, did you spend substantial time obtaining, using it, or recovering from its effects?

d. Did you crave or have a strong desire or urge to use ECIGs?

e. Did you spend less time meeting your responsibilities at work, school, or at home, because of your repeated ECIG use?

f. If your ECIG use caused problems with your

g. Did you use the drug more than once in any situation where you or others were physically at risk

(e.g., driving, riding motorbike, using machinery, etc.)

h. Did you continue to use ECIGs, even though it was clear that ECIGs had caused or worsened psychological or physical problems? 
i. Did you reduce or give up important work, social,

j. Did you need to use ECIGs a lot more in order to get the same effect that you got when you first started using it, or did you get much less effect with continued use of the same amount?

K1: When you cut down on heavy or prolonged use of the drug, did you have any of the following side effects:

(Choose yes if 3 or more of the following)

1. Irritability, frustration, anger

2. anxiety

3. difficulty concentrating

4. increased appetite

5. restlessness

6. feeling depressed

7. difficulty sleeping
No

Yes

Check box if says YES to each of following
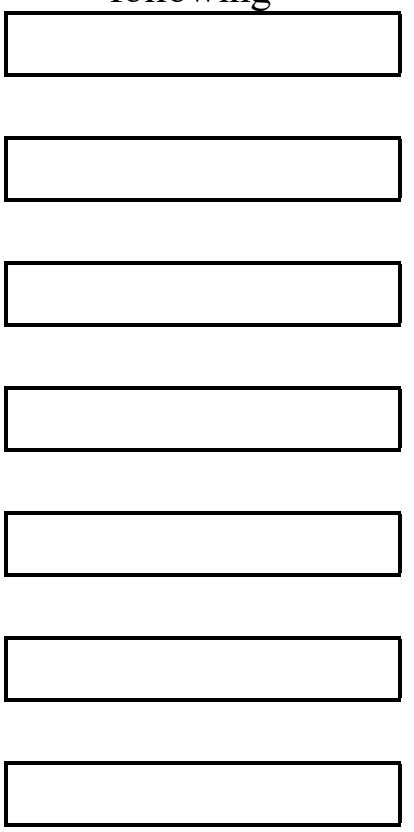

K2: Did you use ECIGs to reduce or avoid withdrawal symptoms?

Yes $=$ ECIG use disorder

No $=$ No disorder 
Severity of SUD (Ja-j, Ksummary)

Mild = 2-3 of symptoms

Moderate $=$ 4-5 symptoms

Severe $=6$ symptoms
Choose 1 Severity

MILD

MODERATE

SEVERE 
Appendix E

Minnesota Nicotine Withdrawal Scale

These phrases may or may not describe how you feel right now. Please respond to each word or phrase with how you feel RIGHT NOW by drawing a vertical mark anywhere along the horizontal line.

Not at all

Extremely

1. URGES to smoke

2. Irritability/frustration/anger

3. Anxious

4. Difficulty concentrating

5. Restlessness

6. Hunger

7. Impatient

8. CRAVING a cigarette/nicotine

9. Drowsiness

10. Depression/feeling blue

11. Desire for sweets
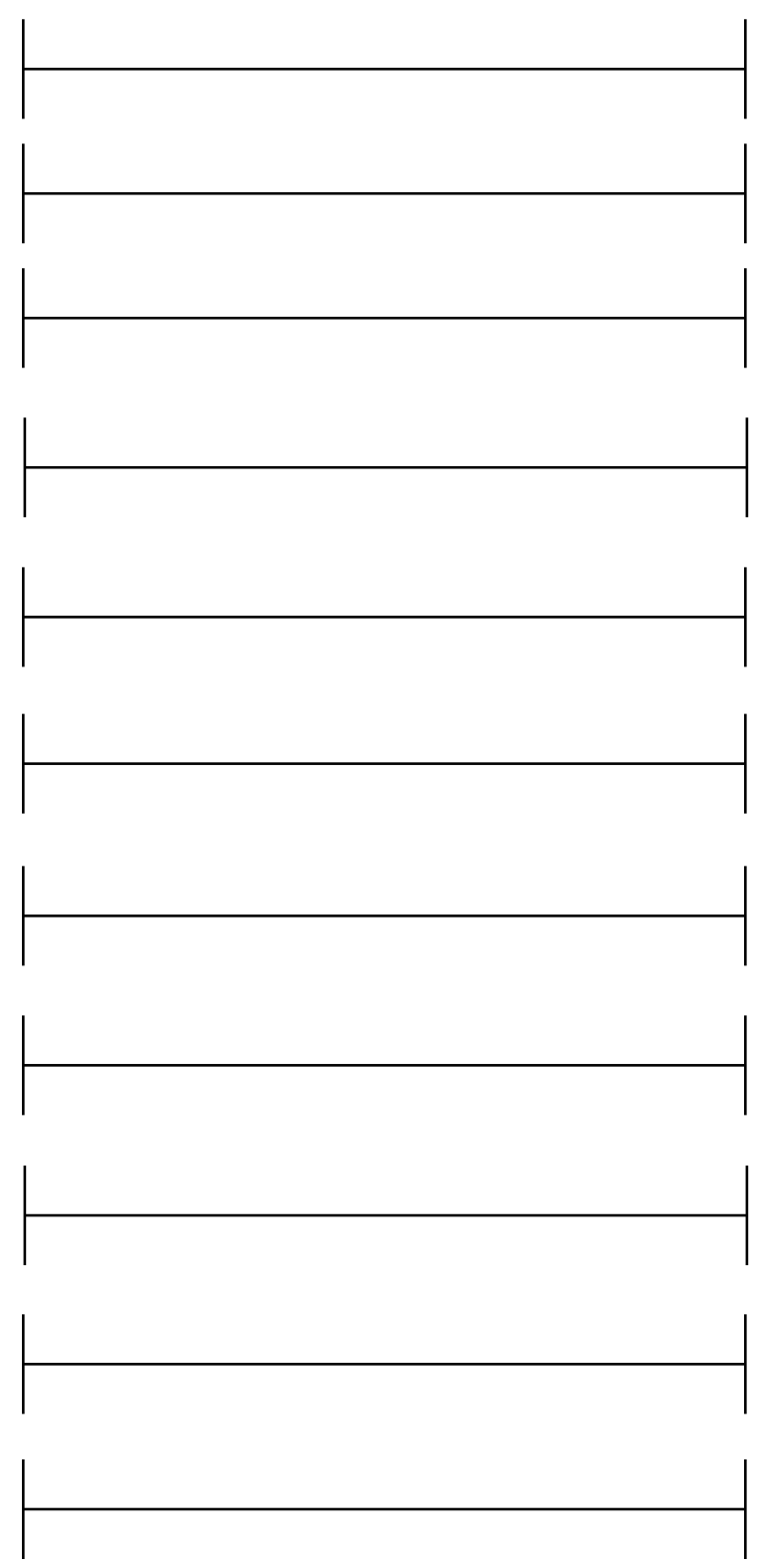
Appendix F

Questionnaire of Smoking Urges - Brief

For each item, please indicate how you feel RIGHT NOW.

1. I have a desire for a cigarette right now.

2. Nothing would be better than smoking a cigarette right now.

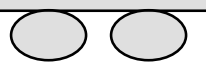

Strongly

disagree
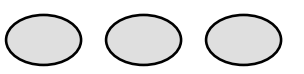

Strongly

disagree
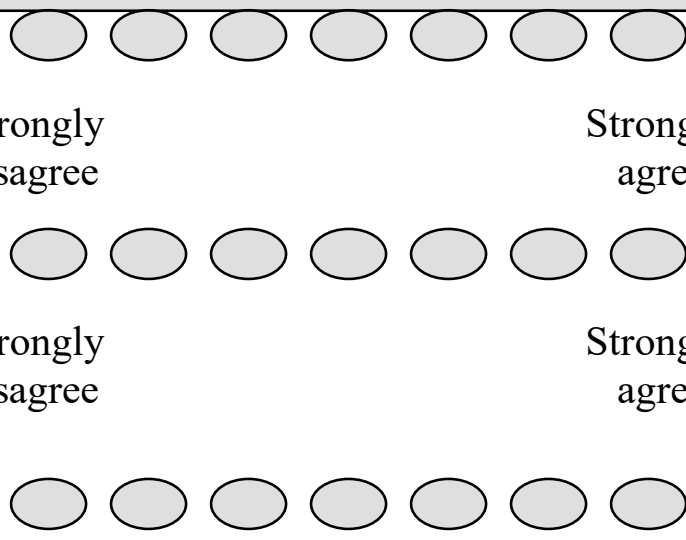

Strongly

disagree

Strongly agree

3. If it were possible, I probably would smoke now.

4. I could control things better right now if I could smoke.

5. All I want right now is a cigarette.

6. I have an urge for a cigarette.

7. A cigarette would taste good now.<smiles>C1CCCCC1</smiles>

Strongly

disagree

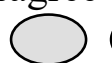

Strongly

disagree

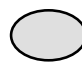

Strongly

disagree

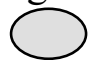

Strongly

disagree

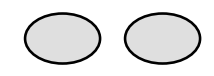

Strongly

disagree

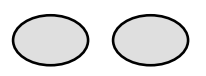

Strongly

disagree

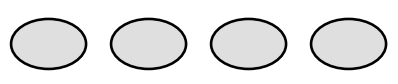

Strongly agree

Strongly agree

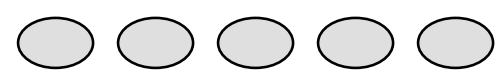

Strongly agree<smiles>[Ge][Ge]1(c2ccccc2)CCCCC1</smiles>

Strongly agree

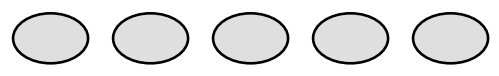

Strongly agree

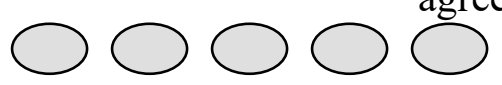

Strongly agree

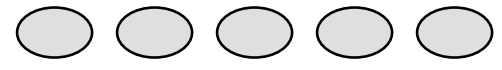

Strongly agree

9. Smoking would make me less depressed.

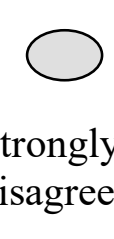

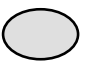

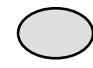

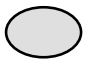

Strongly agree 
10. I am going to smoke as soon as possible.

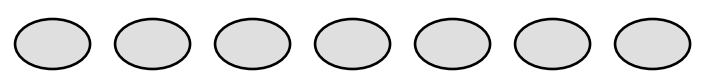

Strongly

Strongly

disagree

agree 


\section{Appendix G}

Direct Effects of Nicotine Scale

These phrases may or may not describe how you feel right now. Please respond to each word or phrase with how you feel RIGHT NOW by drawing a vertical mark anywhere along the horizontal line.

1. Nauseous

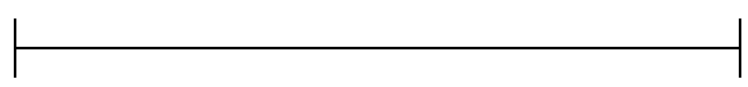

2. Dizzy

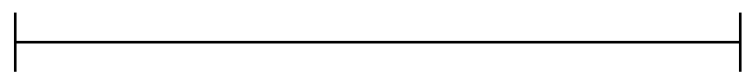

3. Lightheaded

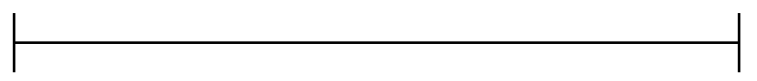

4. Nervous

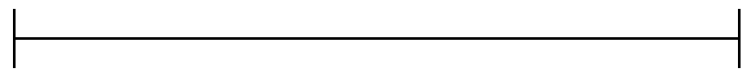

5. Sweaty

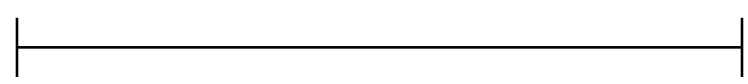

6. Headache

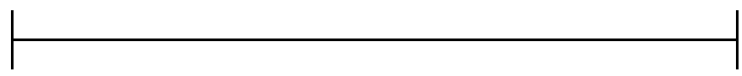

7. Excessive salivation

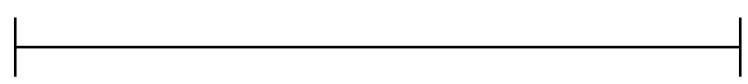

8. Heart pounding

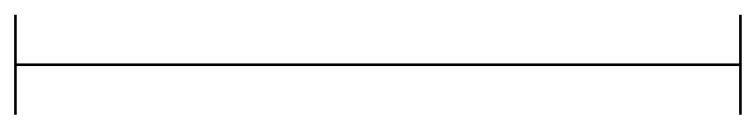

9. Confused

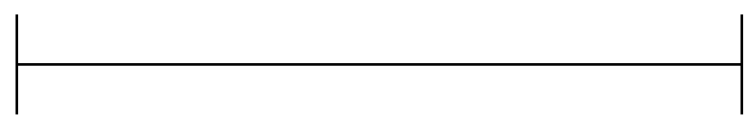

10. Weak

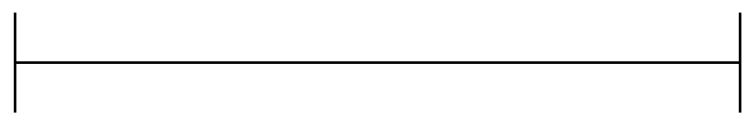




\section{Appendix H}

Direct Effects of Product Scale

These phrases may or may not describe how you feel right now. Please respond to each word or phrase with how you feel RIGHT NOW by drawing a vertical mark anywhere along the horizontal line.

Not at all

1. Was the product satisfying?

2. Was the product pleasant?

3. Did the product taste good?

4. Did the product make you dizzy?

5. Did the product calm you down?

6. Did the product help you concentrate?

7. Did the product make you feel more awake?

8. Did the product reduce your hunger for food?

9. Did the product make you sick?

\section{Extremely}
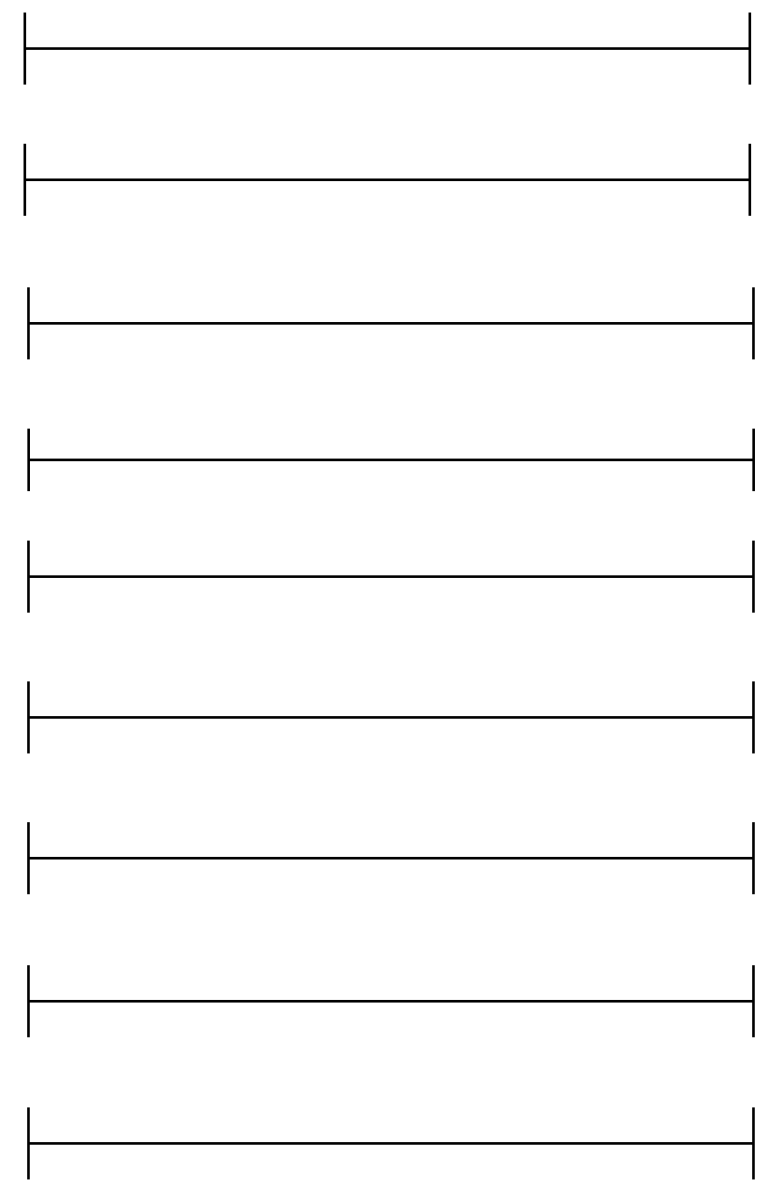
Appendix I

Multiple Choice Procedure

Please choose between the two options.

1. 10 puffs from your ECIG or $\$ 0.01$

2. 10 puffs from your ECIG or $\$ 0.02$

3. 10 puffs from your ECIG or $\$ 0.04$

4. 10 puffs from your ECIG or $\$ 0.08$

5. 10 puffs from your ECIG or $\$ 0.16$

6. 10 puffs from your ECIG or $\$ 0.32$

7. 10 puffs from your ECIG or $\$ 0.64$

8. 10 puffs from your ECIG or $\$ 1.28$

9. 10 puffs from your ECIG or $\$ 2.56$

10. 10 puffs from your ECIG or $\$ 5.12$ 


\section{Appendix J \\ ECIG Dependence Predictor Variables}

Table 5 demonstrates skew, kurtosis, and correlation with PSECDI scores for all variables. The outcome variable, PSECDI scores, was normally distributed though no predictor variables were normally distributed. For predictor variables, first, univariate outliers were truncated to the next lowest score for variables when z-scores $>3.2$ and the reported value may not have been realistic (e.g. wattage of 157 for Juul, which is approximately 8 watts). One to three outliers were truncated for lifetime cigarettes, liquid/day, duration of use, and wattage. Though this improved the distribution of these variables, they were not normally distributed after handling of outliers.

Lifetime cigarette use was highly skewed and kurtotic with a large standard error (Table $\mathrm{X}$ ), so this variable was dichotomized into smoking status. Individuals that had used $\geq 100$ cigarettes lifetime were classified as smokers (27.40\%), and individuals that had used $<100$ lifetime cigarettes were considered nonsmokers $(72.60 \%)$. Nonsmokers $(M=8.47, S E M=0.40)$ and smokers $(M=8.58$, SEM $=0.62)$ did not have significantly different PSEDCI scores, based on an independent-samples $t$-test $[t(166)=-0.16, p=0.88]$. Nicotine concentration was retained for the model because a log transformation worsened skew and kurtosis for this variable (Table 5). One concern for this variable was the low correlation with PSECDI scores. Log and square root transformation worsened skew and kurtosis for days/week (Table 5). Since $87.6 \%$ of respondents used ECIGs daily, days/week was dichotomized into daily and non-daily use for the final model. Daily ECIG users $(M=8.95, S E M=0.36)$ had significantly higher PSEDCI scores than nondaily users $(M=5.52, S E M=0.75)$, based on an independent samples $t$-test $[t(168)=$ $3.40, p=.001]$. Log-transformed liquid/day was used in the model due to improved skew and kurtosis compared to the raw variable. One concern was the potential association between liquid 
use/day and the PSEDCI question "How many times per day do you use your ECIG." However, these variables were not significantly correlated $(r=.07, p=0.36)$, suggesting that liquid use/day could be retained in the model. Square root-transformed duration of use was used in the model due to acceptable skew and kurtosis after transformation. Log-transformed wattage was used in the model due to improved skew and kurtosis, though skew remained outside of the normal range. Log-transformed age was used to the model due to improved skew and kurtosis, despite being outside of the normal range (Table 5).

Additionally, interactions between variables were explored. Specifically, nicotine $\mathrm{x}$ wattage, nicotine use/day (nicotine concentration x liquid use/day) and nicotine use/week (nicotine concentration $\mathrm{x}$ liquid use/day $\mathrm{x}$ days/week) were investigated. Nicotine $\mathrm{x}$ wattage was of interest due to the high correlation between these variables $(r=-.78, p<.001)$. However, these interaction variables had extreme levels of skew and kurtosis and were less normally distributed than the original variables. Additionally, these variables were not significantly correlated with dependence. Finally, the utility of these variables was unclear (e.g., how nicotine concentration and wattage interact to influence nicotine delivery is not well-characterized), so the original variables were determined to be more appropriate for the model. Juul use was considered as a variable rather than nicotine concentration and wattage because $60.74 \%$ of the sample used a Juul. Juul use was not significantly associated with PSECDI scores, $t(168)=.04, p$ $=0.97$, so the original variables were retained for the model. 